\title{
Motives with modulus, I: Modulus sheaves with transfers for non-proper modulus pairs
}

\author{
Bruno Kahn, Hiroyasu Miyazaki, Shuji Saito, and Takao Yamazaki
}

\begin{abstract}
We develop a theory of modulus sheaves with transfers, which generalizes Voevodsky's theory of sheaves with transfers. This paper and its sequel are foundational for the theory of motives with modulus, which is developed in [KMSY20].
\end{abstract}

Keywords. modulus pair; presheaf with transfers; cd-structure

2020 Mathematics Subject Classification. 19E15; 14F42; 19D45; 19F15

[Français]

Motifs avec modules, I : faisceaux avec transferts pour les couples modulaires non propres

Résumé. Nous présentons une théorie de faisceaux modulaires avec transferts qui généralise la théorie des faisceaux avec transferts de Voevodsky. Cet article et celui qui lui fait suite sont les fondements d'une théorie de motifs avec modules, qui est développée dans [KMSY20].

\footnotetext{
Received by the Editors on December 17, 2019, and in final form on November 26, 2020.

Accepted on December 19, 2020.

Bruno Kahn

IMJ-PRG, Case 247, 4 place Jussieu, 75252 Paris Cedex 05, France

e-mail: bruno.kahn@imj-prg.fr

Hiroyasu Miyazaki

RIKEN iTHEMS, Wako, Saitama 351-0198, Japan

e-mail: hiroyasu.miyazaki@riken.jp

Shuji Saito

Graduate School of Mathematical Sciences, University of Tokyo, 3-8-1 Komaba, Tokyo 153-8941, Japan

e-mail: sshuji@msb.biglobe.ne.jp

Takao Yamazaki

Institute of Mathematics, Tohoku University, Aoba, Sendai 980-8578, Japan

e-mail: ytakao@math.tohoku.ac.jp
}

The first author acknowledges the support of Agence Nationale de la Recherche (ANR) under reference ANR-12-BL01-0005. The work of the second author is supported in part by Fondation des Sciences Mathématiques de Paris (FSMP), and in part by RIKEN Special Postdoctoral Researchers (SPDR) Program and RIKEN Interdisciplinary Theoretical and Mathematical Sciences (iTHEMS) Program. The third author is supported by JSPS KAKENHI Grant (15H03606). The fourth author is supported by JSPS KAKENHI Grant (15K04773). 


\section{Contents}

Introduction . . . . . . . . . . . . . . . . . . . . . . . . . . . . . . . . . 2

1. Modulus pairs and admissible correspondences. . . . . . . . . . . . . . . . . . 5

2. Presheaf theory . . . . . . . . . . . . . . . . . . . . . . . . . 19

3. Sheaves on $\underline{M S m}^{\text {fin }}$ and $\underline{M C o r}^{f i n}$. . . . . . . . . . . . . . . . . . . . . . . 23

4. Sheaves on $\underline{M S m}$ and $\underline{M} C o r$. . . . . . . . . . . . . . . . . . . . . . . . . 30

A. Categorical toolbox, $\mathbf{I}$. . . . . . . . . . . . . . . . . . . . . . . . . . . . . . 35

References. . . . . . . . . . . . . . . . . . . . . . . . . . . . . . . . . 45

\section{Introduction}

The aim of this paper is to lay a foundation for a theory of motives with modulus, which will be completed in [KMSY20], generalizing Voevodsky's theory of motives. Voevodsky's construction is based on $\mathbf{A}^{1}$ invariance. It captures many important invariants such as Bloch's higher Chow groups, but not their natural generalisations like additive Chow groups [BE03, Par09] or higher Chow groups with modulus [BS19]. Our basic motivation is to build a theory that captures such non $\mathbf{A}^{1}$-invariant phenomena, as an extension of [KSY16].

Let $\mathrm{Sm}$ be the category of smooth separated schemes of finite type over a field $k$. Voevodsky's construction starts from an additive category Cor, whose objects are those of Sm and morphisms are finite correspondences. We define PST as the category of additive presheaves of abelian groups on Cor (i.e. functors Cor $\rightarrow$ Ab that commute with finite sums). Let NST $\subset$ PST be the full subcategory of those objects $F \in \mathbf{P S T}$ whose restrictions $F_{X}$ to $X_{\mathrm{Nis}}$ is a sheaf for any $X \in \mathbf{S m}$, where $X_{\mathrm{Nis}}$ denotes the small Nisnevich site of $X$, that is, the category of all étale schemes over $X$ equipped with the Nisnevich topology. Objects of NST are called (Nisnevich) sheaves with transfers. For $F \in$ NST, we write

$$
H_{\mathrm{Nis}}^{i}(X, F)=H^{i}\left(X_{\mathrm{Nis}}, F_{X}\right) \text {. }
$$

The following result of Voevodsky [Voe00, Theorem 3.1.4] plays a fundamental rôle in his theory of motives.

Theorem 1 (Voevodsky). The following assertions hold.

(1) The inclusion NST $\rightarrow$ PST has an exact left adjoint $a_{\mathrm{Nis}}^{V}$ such that for any $F \in \mathrm{PST}$ and $X \in \mathrm{Sm}$, $\left(a_{\mathrm{Nis}}^{V} F\right)_{X}$ is the Nisnevich sheafication of $F_{X}$ as a presheaf on $X_{\mathrm{Nis}}$. In particular NST is a Grothendieck abelian category.

(2) For $X \in \mathrm{Sm}$, let $\mathbb{Z}_{\mathrm{tr}}(X)=\operatorname{Cor}(-, X) \in \mathrm{PST}$ be the associated representable additive presheaf. Then we have $\mathbb{Z}_{\mathrm{tr}}(X) \in \mathrm{NST}$ and there is a canonical isomorphism for any $i \geq 0$ and $F \in \mathrm{NST}$ :

$$
H_{\mathrm{Nis}}^{i}(X, F) \simeq \operatorname{Ext}_{\mathrm{NST}}^{i}\left(\mathbb{Z}_{\mathrm{tr}}(X), F\right) .
$$

Our basic principle for generalizing Voevodsky's theory of sheaves with transfers is that the category

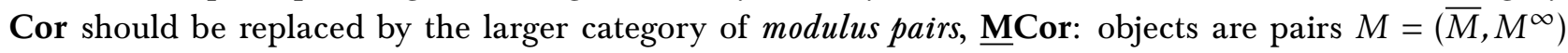
consisting of a separated $k$-scheme of finite type $\bar{M}$ and an effective (possibly empty) Cartier divisor $M^{\infty}$ on it such that the complement $M^{\circ}:=\bar{M} \backslash M^{\infty}$ is smooth over $k$. The group $\underline{\operatorname{MCor}}(M, N)$ of morphisms is 
defined as the subgroup of $\operatorname{Cor}\left(M^{\circ}, N^{\circ}\right)$ consisting of finite correspondences between $M^{\circ}$ and $N^{\circ}$ whose closures in $\bar{M} \times_{k} \bar{N}$ are proper ${ }^{1}$ over $\bar{M}$ and satisfy certain admissibility conditions with respect to $M^{\infty}$ and

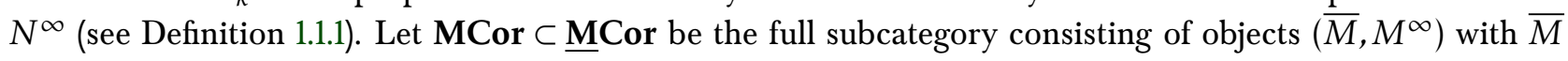
proper over $k$.

We then define MPST (resp. MPST) as the category of additive presheaves of abelian groups on $\underline{\text { MCor }}$ (resp. MCor). We have a functor

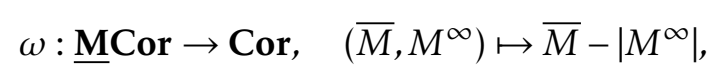

and two pairs of adjunctions

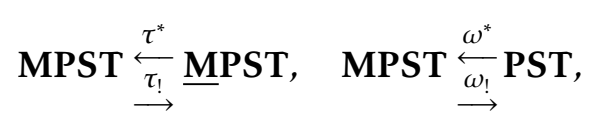

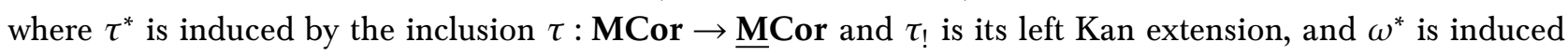
by $\omega$ and $\omega_{\text {! }}$ is its left Kan extension (see Propositions 2.4.1 and 2.2.1).

The main aim of this paper is to develop a sheaf theory on MCor generalizing Voevodsky's theory. For

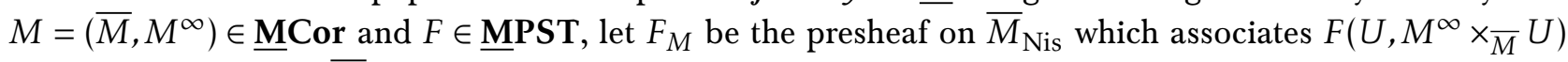
to an étale map $U \rightarrow \bar{M}$.

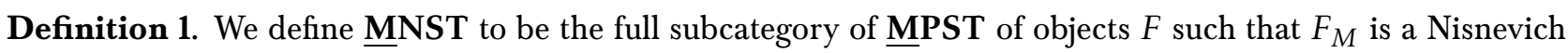
sheaf on $\bar{M}$ for any $M \in \underline{\text { MCor. }}$

For $F \in \underline{\text { MPST }}$ and $M=\left(\bar{M}, M^{\infty}\right)$, let $\left(F_{M}\right)_{\text {Nis }}$ be the Nisnevich sheafication of the preshseaf $F_{M}$ on

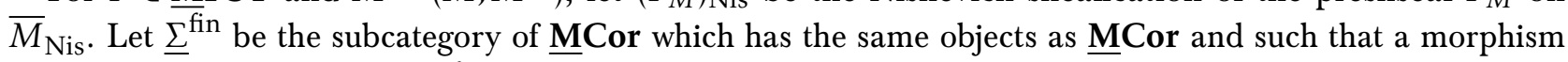
$f \in \underline{\operatorname{MCor}}(M, N)$ belongs to $\underline{\Sigma}^{\mathrm{fin}}$ if and only if $f^{\mathrm{o}} \in \operatorname{Cor}\left(M^{\mathrm{o}}, N^{\mathrm{o}}\right)$ is the graph of an isomorphism $M^{\mathrm{o}} \stackrel{\sim}{\rightarrow} N^{\mathrm{o}}$ in $\mathrm{Sm}$ that extends to a proper morphism $\bar{f}: \bar{M} \rightarrow \bar{N}$ of $k$-schemes such that $M^{\infty}=\bar{f}^{*} N^{\infty}$. (See Theorems 4.5.5, 4.6.3 and Lemma 4.5.3.)

Theorem 2. The following assertions hold.

(1) The inclusion $\underline{\mathrm{MNST}} \rightarrow \underline{\mathrm{MPST}}$ has an exact left adjoint $\underline{a}_{\mathrm{Nis}}$ such that

$$
\left(\underline{a}_{\mathrm{Nis}} F\right)(M)=\underset{N \in \underline{\underline{\Sigma}}^{\mathrm{fin}} \downarrow M}{\lim }\left(F_{N}\right)_{\mathrm{Nis}}(N)
$$

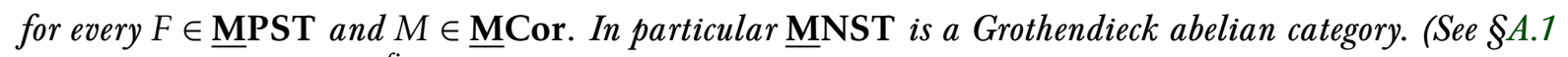
for the comma category $\underline{\Sigma}^{\text {fin }} \downarrow \bar{M}$.)

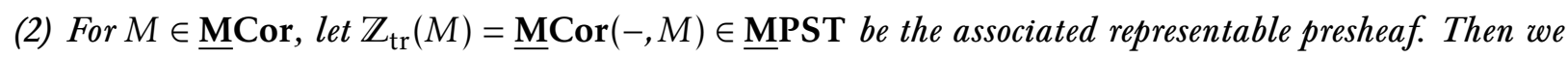
have $\mathbb{Z}_{\mathrm{tr}}(M) \in \underline{\text { MNST }}$ and there is a canonical isomorphism for any $i \geq 0$ and $F \in \underline{\text { MNST: }}$

$$
\operatorname{Ext}_{\underline{\mathrm{MNST}}}^{i}\left(\mathbb{Z}_{\mathrm{tr}}(M), F\right) \simeq \underset{N \in \underline{\Sigma}^{\mathrm{fin}} \downarrow M}{\lim _{\mathrm{Nis}}} H_{\mathrm{Nis}}^{i}\left(\bar{N}, F_{N}\right) .
$$

Remark 1. Theorem 2 (2) describes the extension groups in MNST in terms of classical cohomology. It also implies that the formation

$$
M \mapsto \underset{N \in \underline{\Sigma}_{\text {fin }} \downarrow M}{\lim _{\mathrm{Nis}}} H_{N}^{i}\left(\bar{N}, F_{N}\right)
$$

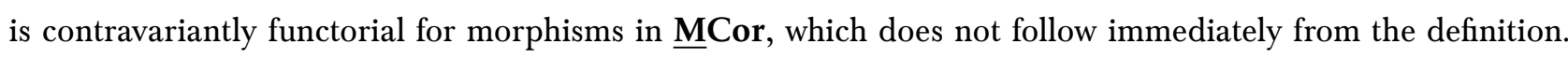

The preprint [KSY15] contained a mistake, pointed out by Joseph Ayoub: namely, Proposition 3.5.3 of loc. cit. is false. Theorem 2 (1) shows that the only false thing in that proposition is that the functor $\underline{b}^{\mathrm{Nis}}$ of loc. cit. is not exact, but only left exact (see Proposition 4.5.4 of the present paper.) This weakens [KSY15, Proposition 3.6.2] into Theorem 2 (2); see however Question 1 below. What we gain in the present correction

\footnotetext{
${ }^{1}$ Here we stress that we do not assume it is finite over $\bar{M}$.
} 


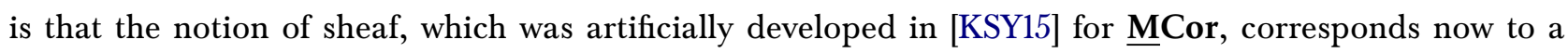
genuine Grothendieck topology.

Another proposition incorrectly proven in [KSY15] was Proposition 3.7.3. In Part II of this work [KMSY21], we correct this proof and recover the proposition in full, hence get a good sheaf theory also for proper modulus pairs. This allows us to develop the categories of motives again in [KMSY20].

In the last part of this introduction, we raise the following question. Its affirmative answer would simplify the right hand side of Theorem 2 (2) under two additional conditions (i) and (ii) below. (These conditions turn out to be essential in [Sai20].)

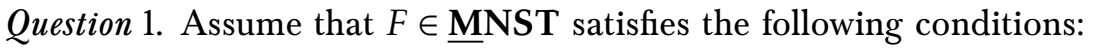

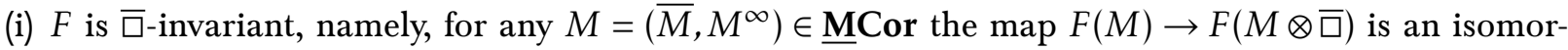
phism, where

$$
\bar{\square}=\left(\mathbf{P}^{1}, \infty\right), \quad M \otimes \bar{\square}=\left(\bar{M} \times \mathbf{P}^{1}, M^{\infty} \times \mathbf{P}^{1}+\bar{M} \times(\infty)\right) .
$$

(ii) $F$ lies in the essential image of $\tau_{!}:$MPST $\rightarrow$ MPST.

Then, is the map

$$
H^{q}\left(\bar{M}_{\mathrm{Nis}}, F_{M}\right) \rightarrow \underset{N \in{\underline{\Sigma^{\mathrm{fin}}} \downarrow M}^{\lim }}{\rightarrow} H^{q}\left(\bar{N}_{\mathrm{Nis}}, F_{N}\right)
$$

an isomorphism for $M \in \underline{\mathbf{M C o r}_{l s}}$ ? Here $\underline{\mathbf{M C o r}}_{l s}$ denotes the full subcategory of $\underline{\mathbf{M}}$ Cor consisting of the objects $M=\left(\bar{M}, M^{\infty}\right)$ such that $\bar{M} \in \mathbf{S m}$ and $\left|M^{\infty}\right|$ is a simple normal crossing divisor on $\bar{M}$.

If $\operatorname{ch}(k)=0$, by resolution of marked ideals ([BM08, the case $d=1$ of Theorem 1.3]), the above question is reduced to the following.

Question 2. Let the assumptions be as in Question 1 and $M=\left(\bar{M}, M^{\infty}\right) \in \underline{\mathbf{M C o r}}_{l s}$. Let $Z \subset M^{\infty}$ be a regular closed subscheme such that, for any point $x$ of $Z$, there exists a system $z_{1}, \ldots, z_{d}$ of regular parameters of $\bar{M}$ at $x$ (with $d=\operatorname{dim}_{x} \bar{M}$ ) satisfying the following conditions:

- Locally at $x, Z=\left\{z_{1}=\cdots=z_{r}=0\right\}$ for $r=\operatorname{codim}_{\bar{M}} Z$.

- Locally at $x,\left|M^{\infty}\right|=\left\{\prod_{j \in J} z_{j}=0\right\}$ for some $J \subset\{1, \ldots, r\}$.

Consider $\pi: \bar{N}=\mathbf{B l}_{Z}(\bar{M}) \rightarrow \bar{M}$ and $N^{\infty}=\bar{N} \times \bar{M} M^{\infty}$. Then, is the map

$$
H^{q}\left(\bar{M}_{\mathrm{Nis}}, F_{M}\right) \rightarrow H^{q}\left(\bar{N}_{\mathrm{Nis}}, F_{N}\right) \text {. }
$$

an isomorphism?

\section{Acknowledgements}

Part of this work was done while the authors stayed at the university of Regensburg supported by the SFB grant "Higher Invariants". Another part was done in a Research in trio in CIRM, Luminy. Yet another part was done while the fourth author was visiting IMJ-PRG supported by Fondation des Sciences Mathématiques de Paris. We are grateful to the support and hospitality received in all places.

We thank Ofer Gabber and Michel Raynaud for their help with Lemma 1.6.1, and Kay Rülling for pointing out an error and correcting Definition 1.8.1.

We are very grateful to Joseph Ayoub for pointing out a flaw on the computation of the sheafification functor $\underline{a}_{\mathrm{Nis}}$ and on the non-exactness of the functor $\underline{b}^{\mathrm{Nis}}$ in the earlier version. The authors believe that the whole theory has been deepened by the effort to fix it. We also thank the referees for a careful reading and many useful comments.

Finally, the influence of Voevodsky's ideas is all-pervasive, as will be evident when reading this paper. 


\section{Notation and conventions}

In the whole paper we fix a base field $k$. Let $S m$ be the category of separated smooth schemes of finite type over $k$, and let Sch be the category of separated schemes of finite type over $k$. We write Cor for Voevodsky's category of finite correspondences [Voe00].

\section{Modulus pairs and admissible correspondences}

\subsection{Admissible correspondences}

\section{Definition 1.1.1.}

(1) A modulus pair $M$ consists of $\bar{M} \in \mathrm{Sch}$ and an effective Cartier divisor $M^{\infty} \subseteq \bar{M}$ such that the open subset $M^{\mathrm{o}}:=\bar{M}-\left|M^{\infty}\right|$ is smooth over $k$. (The case $\left|M^{\infty}\right|=\emptyset$ is allowed.) We say that $M$ is proper if $\bar{M}$ is proper over $k$.

We write $M=\left(\bar{M}, M^{\infty}\right)$, since $M$ is completely determined by the pair, although we regard $M^{\circ}$ as the main part of $M$. We call $\bar{M}$ the ambient space of $M$ and $M^{\circ}$ the interior of $M$.

(2) Let $M_{1}, M_{2}$ be modulus pairs. Let $Z \in \operatorname{Cor}\left(M_{1}^{\mathrm{o}}, M_{2}^{\mathrm{o}}\right)$ be an elementary correspondence (i.e. an integral closed subscheme of $M_{1}^{\mathrm{o}} \times M_{2}^{\mathrm{o}}$ which is finite and surjective over an irreducible component of $\left.M_{1}^{\mathrm{o}}\right)$. We write $\bar{Z}^{N}$ for the normalization of the closure $\bar{Z}$ of $Z$ in $\bar{M}_{1} \times \bar{M}_{2}$ and $p_{i}: \bar{Z}^{N} \rightarrow \bar{M}_{i}$ for the canonical morphisms for $i=1,2$. We say $Z$ is admissible for $\left(M_{1}, M_{2}\right)$ if $p_{1}^{*} M_{1}^{\infty} \geq p_{2}^{*} M_{2}^{\infty}$. An element of $\operatorname{Cor}\left(M_{1}^{\mathrm{o}}, M_{2}^{\mathrm{o}}\right)$ is called admissible if all of its irreducible components are admissible. We write $\operatorname{Cor}_{\text {adm }}\left(M_{1}, M_{2}\right)$ for the subgroup of $\operatorname{Cor}\left(M_{1}^{\mathrm{o}}, M_{2}^{\mathrm{o}}\right)$ consisting of all admissible correspondences.

\section{Remarks 1.1.2.}

(1) In [KSY16, Definition 2.1.1], we used a different notion of modulus pair, where $\bar{M}$ is supposed proper, $M^{\mathrm{o}}$ smooth quasi-affine and $M^{\infty}$ is any closed subscheme of $\bar{M}$. Definition 1.1.1 (1) is the right one for the present work. Definition 1.1.1 (2) is the same as [KSY16, Definition 2.6.1], mutatis mutandis. An analogous condition was considered much earlier in the context of the additive Chow groups (see, e.g. [BE03, (6.4)], [Par09, Definition 2.2], [Rül07, Definition 3.1]).

(2) In the first version of this paper, we imposed the condition that $\bar{M}$ is locally integral; it is now removed. The main reason for this change is that this condition is not stable under products or extension of the base field. The next remark shows that this removal is reasonable (see also Remark 1.3.8).

(3) Let $M$ be a modulus pair. Then $M^{\circ}$ is dense in $\bar{M}$, since the Cartier divisor $M^{\infty}$ is everywhere of codimension 1. Moreover, $\bar{M}$ is reduced. (In particular, $\bar{M}$ has no embedded component.) Indeed, take $x \in \bar{M}$ and let $f \in \mathcal{O}_{\bar{M}, x}$ be a local equation for $M^{\infty}$. Then $f$ is not a zero-divisor (since $M^{\infty}$ is Cartier), and hence $\mathcal{O}_{\bar{M}, x} \rightarrow \mathcal{O}_{\bar{M}, x}[1 / f]$ is injective, but $\mathcal{O}_{\bar{M}, x}[1 / f]$ is reduced as $M^{\mathrm{o}}$ is smooth. In particular, $\bar{M}$ is integral if $M^{\circ}$ is.

(4) Let $M$ be a modulus pair, and let $f: \bar{M}_{1} \rightarrow \bar{M}$ be a morphism such that $f(T) \not \subset\left|M^{\infty}\right|$ for any irreducible component $T$ of $\bar{M}_{1}$ and $M_{1}^{\mathrm{o}}:=\bar{M}_{1}-\left|f^{*} M^{\infty}\right|$ is smooth. Then $M_{1}=\left(\bar{M}_{1}, f^{*} M^{\infty}\right)$ defines a modulus pair. We call it the minimal modulus structure induced by $f$. We shall use this construction several times. Also, $f$ defines a minimal morphism $f: M_{1} \rightarrow M$ in the sense of Definition 1.3.4 below.

(5) If $Z$ is an admissible elementary correspondence as in Definition 1.1.1 (2), then

$$
\left|M_{1}^{\infty} \times \bar{M}_{2}\right| \cap \bar{Z} \supseteq\left|\bar{M}_{1} \times M_{2}^{\infty}\right| \cap \bar{Z}
$$

since $\bar{Z}^{N} \rightarrow \bar{Z}$ is surjective. On the other hand, the inequality $\left.\left(M_{1}^{\infty} \times \bar{M}_{2}\right)\right|_{\bar{Z}} \geq\left.\left(\bar{M}_{1} \times M_{2}^{\infty}\right)\right|_{\bar{Z}}$ may fail. As an example, let $C$ be the affine cusp curve Spec $k[x, y] /\left(x^{2}-y^{3}\right)$. Its normalization is $\mathbf{A}^{1}$, 
via the morphism $t \mapsto\left(t^{3}, t^{2}\right)$. Let $M_{1}=(C,(x))$ and $M_{2}=(C,(y))$. Then $1_{C}$ defines an admissible correspondence $M_{1} \rightarrow M_{2}$, even though $(x) \geq(y)$ does not hold on $C$.

The following lemma will play a key rôle:

Lemma 1.1.3. Let $\bar{X} \in \mathrm{Sch}$ and let $X$ be an open dense subscheme of $\bar{X}$. Assume that $X \in \mathrm{Sm}$ and that $\bar{X}-X$ is the support of a Cartier divisor. Then for any modulus pair $N$ we have

$$
\bigcup_{M} \operatorname{Cor}_{\mathrm{adm}}(M, N)=\operatorname{Cor}\left(X, N^{\mathrm{o}}\right),
$$

where $M$ ranges over all modulus pairs such that $\bar{M}=\bar{X}$ and $M^{\circ}=X$. (Note that by definition we have $\operatorname{Cor}_{\mathrm{adm}}(M, N) \subset \operatorname{Cor}\left(X, N^{\mathrm{o}}\right)$.)

Proof. This is proven in [KSY16, Lemma 2.6.2]. In loc. cit. $X$ and $N^{\circ}$ are assumed to be quasi-affine, and $\bar{X}$ and $\bar{N}$ proper and normal (see Remark 1.1.2). But these assumptions are not used in the proof. (Nor is the assumption on Cartier divisors, but the latter is essential for the proof of Proposition 1.2.4 below.)

\subsection{Composition}

To discuss composability of admissible correspondences, we need the following lemma of Krishna and Park [KP12, Lemma 2.2].

Lemma 1.2.1. Let $f: X \rightarrow Y$ be a surjective morphism of normal integral schemes, and let $D, D^{\prime}$ be two Cartier divisors on $Y$. If $f^{*} D^{\prime} \leq f^{*} D$, then $D^{\prime} \leq D$.

We also need the following "containment lemma" from [KP12, Proposition 2.4], [BS19, Lemma 2.1], [Miy19, Lemma 2.4]. We provide a proof for self-containedness.

Lemma 1.2.2. Let $M=\left(\bar{M}, M^{\infty}\right)$ be a modulus pair. Let $V^{\prime} \subset V \subset M^{\circ}=\bar{M}-\left|M^{\infty}\right|$ be two integral closed subschemes. Let $\bar{V}$ and $\overline{V^{\prime}}$ be their closures in $\bar{M}$ and $\bar{V}^{N} \rightarrow \bar{V},{\overline{V^{\prime}}}^{N} \rightarrow \overline{V^{\prime}}$ the normalizations. If $\left.M^{\infty}\right|_{\bar{V}^{N}}$ is effective, so is $\left.M^{\infty}\right|_{\bar{V}^{\prime}}$.

Proof. Set $Z:=\bar{V}^{N} \times \bar{V} \overline{V^{\prime}}$ and consider the following commutative diagram:

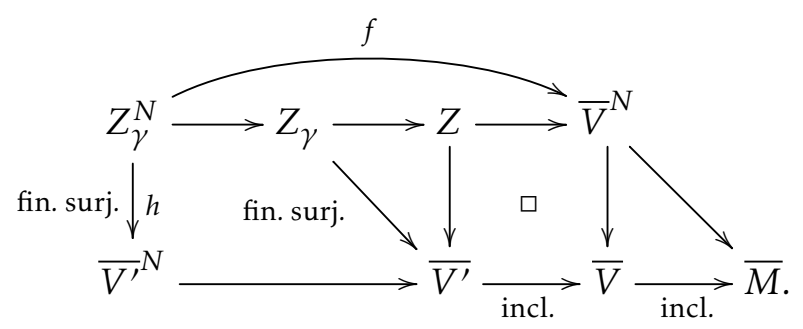

Here, $Z_{\gamma} \subset Z$ is an irreducible component of $Z$ such that the composite map

$$
Z_{\gamma} \hookrightarrow Z \rightarrow \overline{V^{\prime}}
$$

is finite surjective. To see that such a $Z_{\gamma}$ exists, it suffices to note that $\bar{V}^{N} \rightarrow \bar{V}$ is finite surjective, hence so is its base change $Z \rightarrow \overline{V^{\prime}}$ (recall that for any scheme $S$ of finite type over $k$, the normalization $S^{N} \rightarrow S$ is a finite surjective morphism). Then $Z_{\gamma}^{N}$ is also irreducible. Since $Z_{\gamma}^{N} \rightarrow \overline{V^{\prime}}$ is dominant, the vertical map $h$ on the left exists by the universal property of normalization, and is finite surjective. Note that we can pullback the Cartier divisor $M^{\infty}$ to any scheme except for $Z$ in the diagram, since none of their irreducible components maps into the support $\left|M^{\infty}\right| \subset \bar{M}$. Since the pullback of an effective Cartier divisor is effective, the assumption that $\left.M^{\infty}\right|_{\bar{V}^{N}}$ is effective implies that

$$
f^{*}\left(\left.M^{\infty}\right|_{\bar{V}^{N}}\right)=\left.M^{\infty}\right|_{Z_{\gamma}^{N}}=h^{*}\left(\left.M^{\infty}\right|_{\bar{V}^{\prime}}\right)
$$

is effective. By Lemma 1.2.1, $\left.M^{\infty}\right|_{\bar{V}^{N}}$ is effective since $h$ is surjective. This finishes the proof. 
Definition 1.2.3. Let $M_{1}, M_{2}, M_{3}$ be three modulus pairs, and let us consider $\alpha \in \operatorname{Cor}_{\text {adm }}\left(M_{1}, M_{2}\right)$ and $\beta \in \operatorname{Cor}_{\mathrm{adm}}\left(M_{2}, M_{3}\right)$. We say that $\alpha$ and $\beta$ are composable if their composition $\beta \alpha$ in $\operatorname{Cor}\left(M_{1}^{\mathrm{o}}, M_{3}^{\mathrm{o}}\right)$ is admissible.

Proposition 1.2.4. With the above notations, assume $\alpha$ and $\beta$ are integral and let $\bar{\alpha}$ and $\bar{\beta}$ be their closures in $\bar{M}_{1} \times \bar{M}_{2}$ and $\bar{M}_{2} \times \bar{M}_{3}$ respectively. Then $\alpha$ and $\beta$ are composable provided the projection $\bar{\alpha} \times \bar{M}_{2} \bar{\beta} \rightarrow \bar{M}_{1} \times \bar{M}_{3}$ is proper. This happens in the following cases:

(i) $\bar{\alpha} \rightarrow \bar{M}_{1}$ is proper.

(ii) $\bar{\beta} \rightarrow \bar{M}_{3}$ is proper.

(iii) $\bar{M}_{2}$ is proper over $k$.

Proof. Note that $\alpha \times_{M_{2}^{\circ}} \beta$ is a closed subscheme of $\left(M_{1}^{\mathrm{o}} \times M_{2}^{\mathrm{o}}\right) \times_{M_{2}^{\mathrm{o}}}\left(M_{2}^{\mathrm{o}} \times M_{3}^{\mathrm{o}}\right)=M_{1}^{\mathrm{o}} \times M_{2}^{\mathrm{o}} \times M_{3}^{\mathrm{o}}$; we have $|\beta \alpha|=\left|p_{13 *}\left(\alpha \times M_{2}^{\mathrm{o}} \beta\right)\right|$ where $p_{13}: M_{1}^{\mathrm{o}} \times M_{2}^{\mathrm{o}} \times M_{3}^{\mathrm{o}} \rightarrow M_{1}^{\mathrm{o}} \times M_{3}^{\mathrm{o}}$ is the projection. Let $\gamma$ be a component of $\alpha \times_{M_{2}^{\circ}} \beta$. We have a commutative diagram

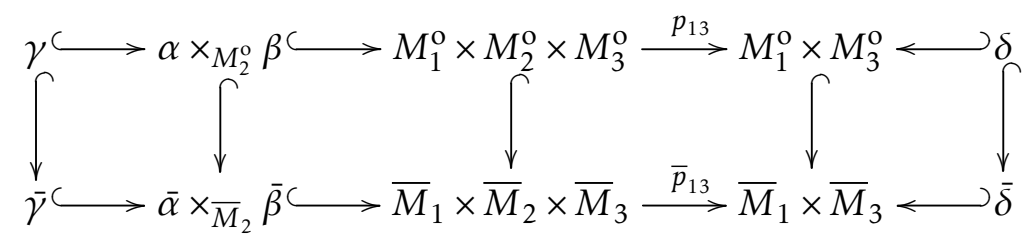

where $\bar{p}_{i j}: \bar{M}_{1} \times \bar{M}_{2} \times \bar{M}_{3} \rightarrow \bar{M}_{i} \times \bar{M}_{j}$ denotes the projection, $\delta=p_{13}(\gamma)$, and ${ }^{-}$denotes closure. The hypothesis implies that $\bar{\gamma} \rightarrow \bar{\delta}$ is proper surjective. The same holds for $\pi_{\gamma \delta}^{N}$ appearing in the second of the two other commutative diagrams:

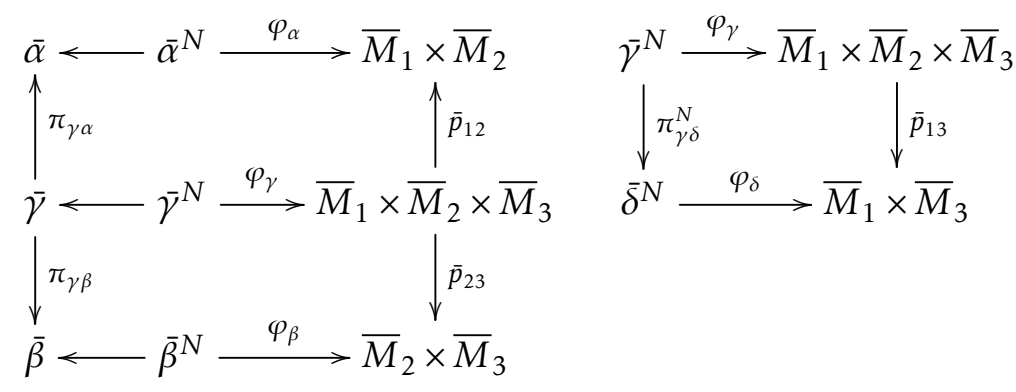

where ${ }^{N}$ means normalisation. (Note that $\pi_{\gamma \alpha}$ and $\pi_{\gamma \beta}$ need not extend to the normalisations, as they need not be dominant.) We have the admissibility conditions for $\alpha$ and $\beta$ :

$$
\begin{aligned}
& \varphi_{\alpha}^{*}\left(\bar{M}_{1} \times M_{2}^{\infty}\right) \leq \varphi_{\alpha}^{*}\left(M_{1}^{\infty} \times \bar{M}_{2}\right) \\
& \varphi_{\beta}^{*}\left(\bar{M}_{2} \times M_{3}^{\infty}\right) \leq \varphi_{\beta}^{*}\left(M_{2}^{\infty} \times \bar{M}_{3}\right) .
\end{aligned}
$$

Applying $^{2}$ Lemma 1.2.2, we get inequalities

$$
\varphi_{\gamma}^{*}\left(\bar{M}_{1} \times \bar{M}_{2} \times M_{3}^{\infty}\right) \leq \varphi_{\gamma}^{*}\left(\bar{M}_{1} \times M_{2}^{\infty} \times \bar{M}_{3}\right) \leq \varphi_{\gamma}^{*}\left(M_{1}^{\infty} \times \bar{M}_{2} \times \bar{M}_{3}\right),
$$

which implies by the right half of the above diagram

$$
\left(\pi_{\gamma \delta}^{N}\right)^{*} \varphi_{\delta}^{*}\left(\bar{M}_{1} \times M_{3}^{\infty}\right) \leq\left(\pi_{\gamma \delta}^{N}\right)^{*} \varphi_{\delta}^{*}\left(M_{1}^{\infty} \times \bar{M}_{3}\right)
$$

hence $\varphi_{\delta}^{*}\left(\bar{M}_{1} \times M_{3}^{\infty}\right) \leq \varphi_{\delta}^{*}\left(M_{1}^{\infty} \times \bar{M}_{3}\right)$ by Lemma 1.2.1.

Finally, one trivially checks that (i) or (ii) implies that the projection $\bar{\alpha} \times \bar{M}_{2} \bar{\beta} \rightarrow \bar{M}_{1} \times \bar{M}_{3}$ is proper, and that (iii) implies both of (i) and (ii).

\footnotetext{
${ }^{2}$ To apply this lemma, factor $\pi_{\gamma \alpha}$ and $\pi_{\gamma \beta}$ into dominant morphisms followed by closed immersions.
} 
Example 1.2.5. Let $\bar{M}_{1}=\bar{M}_{3}=\mathbf{P}^{1}, \bar{M}_{2}=\mathbf{A}^{1}, \bar{M}_{i}^{\mathrm{o}}=\mathbf{A}^{1}, M_{1}^{\infty}=\infty, M_{2}^{\infty}=0, M_{3}^{\infty}=2 \cdot \infty$, and $\alpha=\beta=$ graph of the identity on $\mathbf{A}^{1}$. Then $\alpha$ and $\beta$ are admissible but $\beta \circ \alpha$ is not admissible because $\infty \geq 2 \cdot \infty$ does not hold. (Note that neither of $\bar{\alpha}=\alpha$ or $\bar{\beta}=\beta$ is proper over $\mathbf{P}^{1}$.)

Definition 1.2.6. Let $M, N$ be two modulus pairs. A correspondence $\alpha \in \operatorname{Cor}\left(M^{\circ}, N^{\mathrm{o}}\right)$ is left proper (relatively to $M, N$ ) if the closures of all components of $\alpha$ are proper over $\bar{M}$; this is automatic if $\bar{N}$ is proper.

Proposition 1.2.7. Let $M_{1}, M_{2}, M_{3}$ be three modulus pairs and let $\alpha \in \operatorname{Cor}\left(M_{1}^{\mathrm{o}}, M_{2}^{\mathrm{o}}\right), \beta \in \operatorname{Cor}\left(M_{2}^{\mathrm{o}}, M_{3}^{\mathrm{o}}\right)$ be left proper. Then $\beta \alpha$ is left proper.

Proof. We may assume $\alpha$ and $\beta$ are irreducible. The assumption on $\beta$ means $\bar{\beta} \rightarrow \bar{M}_{2}$ is proper, hence so is its base change $\bar{\alpha} \times \bar{M}_{2} \bar{\beta} \rightarrow \bar{\alpha}$. The assumption on $\alpha$ means $\bar{\alpha} \rightarrow \bar{M}_{1}$ is proper, hence so is $\bar{\alpha} \times \bar{M}_{2} \bar{\beta} \rightarrow \bar{M}_{1}$ as a composition of proper morphisms. This implies the left properness of $\beta \alpha$, since $\overline{\beta \alpha}$ is the image of $\bar{\alpha} \times \bar{M}_{2} \bar{\beta}$ in $\bar{M}_{1} \times \bar{M}_{3}$.

\subsection{Categories of modulus pairs}

Definition 1.3.1. By Propositions 1.2.4 and 1.2.7, modulus pairs and left proper admissible correspondences

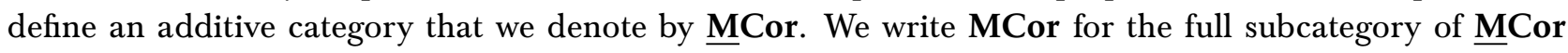
whose objects are proper modulus pairs (see Definition 1.1.1 (1)).

In the context of modulus pairs, the category $\mathbf{S m}$ and the graph functor $\mathrm{Sm} \rightarrow$ Cor are replaced by the following:

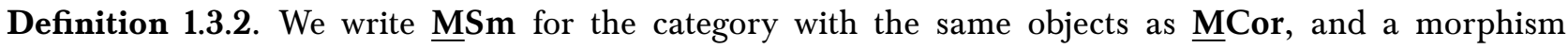
of $\underline{\operatorname{MSm}}\left(M_{1}, M_{2}\right)$ is given by a (scheme-theoretic) $k$-morphism $f: M_{1}^{\mathrm{o}} \rightarrow M_{2}^{\mathrm{o}}$ whose graph belongs to $\underline{\operatorname{MCor}}\left(M_{1}, M_{2}\right)$. We write MSm for the full subcategory of $\underline{\mathbf{M S m}}$ whose objects are proper modulus pairs.

We will need some variants of these categories.

\section{Definition 1.3.3.}

(1) We write $\underline{\mathbf{M C}} \mathrm{r}^{\text {fin }}$ for the subcategory of $\underline{\mathrm{MC}}$ Cor with the same objects and the following condition on

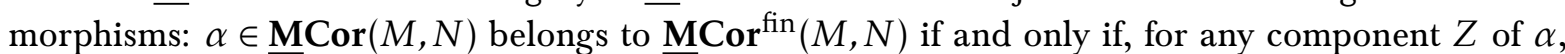
the projection $\bar{Z} \rightarrow \bar{M}$ is finite, where $\bar{Z}$ is the closure of $Z$ in $\bar{M} \times \bar{N}$. The same argument as in the proof of Proposition 1.2.7 shows that $\underline{\mathbf{M} C o r^{\text {fin }}}$ is indeed a subcategory of $\underline{\text { MCor. We write MCor }}{ }^{\text {fin }}$

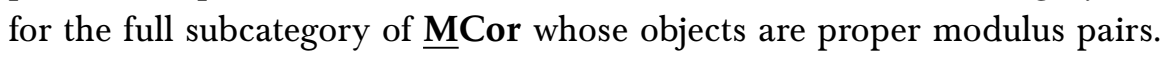

(2) We write $\underline{M S m}^{\text {fin }}$ for the subcategory of $\underline{\mathbf{M S m}}$ with the same objects and such that a morphism $f: M \rightarrow N$ belongs to $\underline{\mathbf{M S m}}{ }^{\text {fin }}$ if and only if $f^{\mathrm{o}}: M^{\mathrm{o}} \rightarrow N^{\mathrm{o}}$ extends to a $k$-morphism $\bar{f}: \bar{M} \rightarrow \bar{N}$. Such extension $\bar{f}$ is unique because $M^{\mathrm{o}}$ is dense in the reduced scheme $\bar{M}$ and $\bar{N}$ is separated ([Har77, Chapter II, Exercise 4.2]). This yields a forgetful functor $\underline{\mathbf{M S m}}^{\text {fin }} \rightarrow \mathbf{S c h}$, which sends $M$ to $\bar{M}$.

We write $\mathbf{M S m}^{\text {fin }}$ for the full subcategory of $\underline{\mathbf{M S m}}$ whose objects are proper modulus pairs.

(3) We write

$$
\begin{aligned}
& c: \text { MSm } \rightarrow \text { MCor }, \\
& \underline{c}: \underline{\text { MSm }} \rightarrow \underline{\text { MCor }}, \\
& \underline{c}^{\text {fin }}: \underline{\mathbf{M S m}}{ }^{\text {fin }} \rightarrow \underline{\mathbf{M}} \text { Cor }^{\text {fin }}
\end{aligned}
$$

for the functors which are the identity on objects and which carry a morphism $f$ to the graph of $f^{\circ}$.

Let $f: M \rightarrow N$ be a morphism in $\underline{\mathbf{M S m}}{ }^{\text {fin }}$. Since $\bar{f}\left(M^{\mathrm{o}}\right) \subseteq N^{\mathrm{o}}$, none of the images of the generic points of the irreducible components of $\bar{M}$ is contained in $\left|N^{\infty}\right|$, hence the pullback of the Cartier divisor $\bar{f}^{*} N^{\infty}$ is well-defined. For ease of notation, we simply write it $f^{*} N^{\infty}$. 
Definition 1.3.4. A morphism $f: M \rightarrow N$ in $\underline{\mathbf{M S m}}^{\text {fin }}$ is minimal if we have $f^{*} N^{\infty}=M^{\infty}$.

Remark 1.3.5. We remark the following.

(1) Assume that $\bar{M}$ is normal. Then Zariski's connectedness theorem implies that for any $N$

$$
\underline{\operatorname{MS}} \mathbf{m}(M, N) \cap \underline{\operatorname{MCor}}^{\mathrm{fin}}(M, N)=\underline{\mathbf{M S m}}^{\mathrm{fin}}(M, N) .
$$

(Indeed, given an elementary correspondence belonging to the left hand side, its closure in $\bar{M} \times \bar{N}$ is birational and finite over an irreducible component of $\bar{M}$, but such a morphism is an isomorphism if $\bar{M}$ is normal by [EGA3, corollaire 4.4.9]). If $f^{\circ}: M^{\mathrm{o}} \rightarrow N^{\mathrm{o}}$ extends to a morphism between ambient spaces $\bar{f}: \bar{M} \rightarrow \bar{N}$, then the graph of $f^{\circ}$ is admissible if and only if we have $M^{\infty} \geq \bar{f}^{*} N^{\infty}$.

(2) For $M \in \underline{\mathbf{M S}} \mathbf{m}^{\text {fin }}$, set $M^{N}:=\left(\bar{M}^{N},\left.M^{\infty}\right|_{\bar{M}^{N}}\right)$ where $p: \bar{M}^{N} \rightarrow \bar{M}$ is the normalization and $\left.M^{\infty}\right|_{\bar{M}^{N}}$ is the pull-back of $M^{\infty}$ to $\bar{M}^{N}$. Then $p: M^{N} \rightarrow M$ is an isomorphism in $\underline{\text { MCor }}{ }^{\text {fin }}$ and $\underline{\text { MSm (but }}$ not in $\underline{\mathbf{M S}} \mathbf{m}^{\text {fin }}$ in general).

(3) Let $M=\left(\bar{M}, M^{\infty}\right)$ and $N=\left(\bar{N}, N^{\infty}\right)$ be two modulus pairs and let $\bar{Z} \subset \bar{M} \times \bar{N}$ be an integral closed subscheme which is finite and surjective over an irreducible component of $\bar{M}$, such that $\bar{Z} \not \subset \bar{M} \times N^{\infty}$ and that $\left.M^{\infty}\right|_{\bar{Z}^{N}} \geq\left. N^{\infty}\right|_{\bar{Z}^{N}}$, where $\bar{Z}^{N}$ is the normalization of $\bar{Z}$. Then $Z=\bar{Z} \cap\left(M^{\mathrm{o}} \times \bar{N}\right)$ belongs to $\operatorname{Cor}\left(M^{\mathrm{o}}, N^{\mathrm{o}}\right)$ and its closure in $\bar{M} \times \bar{N}$ is $\bar{Z}$ : this follows from Remark 1.1.2 (4).

(4) For any morphism $f: M \rightarrow N$ in $\underline{\mathbf{M S m}}$, there exists a morphism $M^{\prime} \rightarrow M$ in $\underline{\mathbf{M S m}}^{\text {fin }}$ which is invertible in $\underline{\mathbf{M S m}}$ such that the induced morphism $M^{\prime} \rightarrow N$ is in $\underline{\mathbf{M S m}}{ }^{\text {fin }}$. More generally, we have the following lemma.

Lemma 1.3.6 (The graph trick). Let $f: M \rightarrow N$ be a morphism in $\underline{\mathrm{MSm}}$. Then there exists a minimal morphism $p: M_{1} \rightarrow M$ in $\underline{M S m}^{\text {fin }}$ such that it is invertible in $\underline{\mathrm{MSm}}$ and the composite $f \circ p: M_{1} \rightarrow M \rightarrow N$ is a morphism in MSm $^{\text {fin }}$. Moreover, if $f^{\mathrm{o}}: M^{\mathrm{o}} \rightarrow N^{\mathrm{o}}$ extends to a morphism $\bar{U} \rightarrow \bar{N}$ for an open subset $\bar{U} \subset \bar{M}$, then we can choose $M_{1}$ such that $\bar{M}_{1} \rightarrow \bar{M}$ is an isomorphism over $\bar{U}$ (note that we can always take $\bar{U}=M^{\circ}$ ).

Proof. Let $\Gamma$ be the graph of the morphism $\bar{U} \rightarrow \bar{N}$, and let $\bar{\Gamma}$ be its closure in $\bar{M} \times \bar{N}$. Then we have natural projections $p_{1}: \bar{\Gamma} \rightarrow \bar{M}$ and $p_{2}: \bar{\Gamma} \rightarrow \bar{N}$. Since we have $\Gamma \cong \bar{U}$, Lemma 1.3.7 below implies that $p_{1}$ is an isomorphism over $\bar{U}$ and we have $p_{1}^{-1}(\bar{U})=\Gamma$. Defining $M_{1}:=\left(\bar{\Gamma}, p_{1}^{*} M^{\infty}\right)$, the morphism $p_{1}$ induces a morphism $p_{1}: M_{1} \rightarrow M$ in $\underline{\mathbf{M S m}}^{\text {fin }}$ such that $f \circ p_{1}: M_{1} \rightarrow M \rightarrow N$ comes from $\underline{\mathbf{M S m}}{ }^{\text {fin }}$ defined by $p_{2}$. Also note that $\bar{\Gamma} \rightarrow \bar{M}$ is proper since $f$ is, which implies that $p_{1}: M_{1} \rightarrow M$ is an isomorphism in $\underline{\mathbf{M S m}}$. This finishes the proof.

Lemma 1.3.7 (No extra fibre lemma). Let $f: X \rightarrow Y$ be a separated morphism of schemes, and let $U \subset X$ be an open dense subset. Assume that the image $f(U)$ of $U$ is open in $Y$, and the induced morphism $U \rightarrow f(U)$ is proper (e.g., an isomorphism). Then, we have $f^{-1}(f(U))=U$.

Proof. Consider the commutative diagram

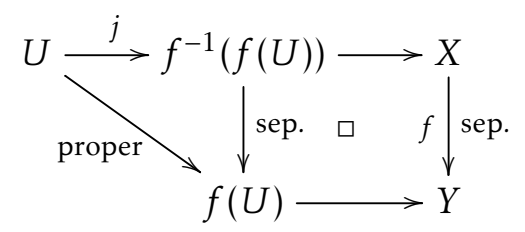

where all the horizontal arrows are open immersions, the square is cartesian and the two vertical morphisms are separated. The triangle diagram on the left implies that $j$ is proper ([Har77, Chapter II, Corollary 4.8]), hence it is a closed (and open) immersion. Since $U$ is dense in $X$, it is dense in $f^{-1}(f(U))$ as well, hence the conclusion. 
Remark 1.3.8. Let $M \in \underline{\text { MCor }}{ }^{\text {fin }}$. Assume that $M^{\mathrm{o}}=M_{1}^{\mathrm{o}} \amalg M_{2}^{\mathrm{o}}$; let $\bar{M}_{i}$ be the closure of $M_{i}^{\mathrm{o}}$ in $\bar{M}$ and $M_{i}^{\infty}$ be the pull-back of $M^{\infty}$ to $\bar{M}_{i}$. Then $M_{i}=\left(\bar{M}_{i}, M_{i}^{\infty}\right)$ are modulus pairs, the inclusions $M_{i}^{\text {o }} \hookrightarrow M^{\mathrm{o}}$ yield morphisms $M_{i} \rightarrow M$ in $\underline{\mathbf{M S m}}^{\text {fin }}$, and the induced morphism in $\underline{\mathbf{M C o r}}{ }^{\mathrm{fin}}$

$$
M_{1} \oplus M_{2} \rightarrow M
$$

is an isomorphism in $\underline{\mathbf{M C o r}}{ }^{\mathrm{fin}}$. The proof is easy and left to the reader.

This remark may help in reducing some reasonings to the case where $M^{\circ}$ is irreducible.

\subsection{The functors $(-)^{(n)}$}

Definition 1.4.1. Let $n \geq 1$ and $M=\left(\bar{M}, M^{\infty}\right) \in \underline{\text { MCor. We write }}$

$$
M^{(n)}=\left(\bar{M}, n M^{\infty}\right) .
$$

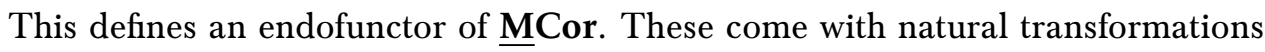

$$
M^{(n)} \rightarrow M^{(m)} \quad \text { if } m \leq n .
$$

Lemma 1.4.2. The functor $(-)^{(n)}$ is fully faithful.

Proof. This follows from the definition and the fact that if $A$ is an integral domain with quotient field $K$, then $a \in K$ is integral over $A$ if and only if so is $a^{n}$.

\subsection{Changes of categories}

We now have a basic diagram of additive categories and functors

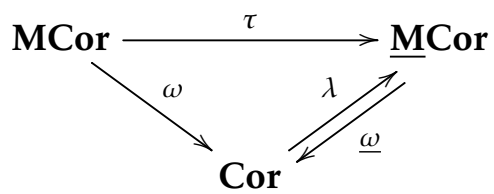

with

$$
\tau(M)=M ; \quad \omega(M)=M^{\mathrm{o}} ; \quad \underline{\omega}(M)=M^{\mathrm{o}} ; \quad \lambda(X)=(X, \emptyset) .
$$

All these functors are faithful, and $\tau$ is fully faithful; they "restrict" to analogous functors $\tau_{s}, \omega_{s}, \underline{\omega}_{s}, \lambda_{s}$ between MSm, $\underline{\mathbf{M S}}$ m and Sm. Note that $\underline{\omega} \circ(-)^{(n)}=\underline{\omega}$ for any $n$. Moreover:

Lemma 1.5.1. We have $\underline{\omega} \tau=\omega$. Moreover, $\lambda$ is left adjoint to $\underline{\omega}$, and the restriction of $\lambda$ to Cor $^{\text {prop }}$ (finite correspondences on smooth proper schemes over $k)$ is "right adjoint" to $\underline{\omega}$. (i.e., $\operatorname{Cor}(\underline{\omega}(M), X)=\underline{\operatorname{MCor}}(M, \lambda(X))$

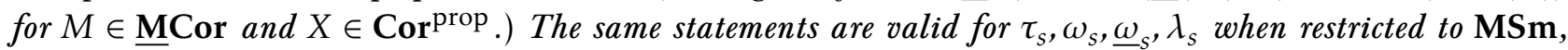
MSm and Sm.

Proof. The first identity is obvious. For the adjointness, let $X \in \operatorname{Cor}, M \in \underline{\mathbf{M C o r}}$ and $\alpha \in \operatorname{Cor}\left(X, M^{\circ}\right)$ be an integral finite correspondence. Then $\alpha$ is closed in $X \times \bar{M}$, since it is finite over $X$ and $\bar{M}$ is separated; it is evidently finite (hence proper) over $X$. It also satisfies $q^{*} M^{\infty}=0$ where $q$ is the composition $\alpha^{N} \rightarrow \alpha \rightarrow M^{\circ} \rightarrow \bar{M}$, because $\left.M^{\infty}\right|_{M^{\circ}}=0$. Therefore $\alpha \in \underline{\operatorname{MCor}}(\lambda(X), M)$.

For the second statement, assume $X$ proper and let $\beta \in \operatorname{Cor}\left(M^{0}, X\right)$ be an integral finite correspondence. Then $\beta$ is trivially admissible, and its closure in $\bar{M} \times X$ is proper over $\bar{M}$, so $\beta \in \underline{\operatorname{MCor}}(M, \lambda(X))$. The last claim is immediate.

The following theorem is an important refinement of Lemma 1.5.1. The proof starts from $\$ 1.7$ and is completed in $\$ 1.8$.

Theorem 1.5.2. The functors $\omega, \tau, \omega_{s}$ and $\tau_{s}$ have pro-left adjoints $\omega^{!}, \tau^{!}, \omega_{s}^{!}$and $\tau_{s}^{!}$(see $\left.\$ A .2\right)$.

General definitions and results on pro-objects and pro-adjoints are gathered in $\S \S A .1$ and A.2. We shall freely use results from there. 


\subsection{The closure of a finite correspondence}

We shall need the following result for the proof of Theorem 1.5.2.

Lemma 1.6.1. Let $X$ be a Noetherian scheme, $\left(\pi_{i}: Z_{i} \rightarrow X\right)_{1 \leq i \leq n}$ a finite set of proper surjective morphisms with $Z_{i}$ integral, and let $U \subseteq X$ be a normal open subset. Suppose that $\pi_{i}: \pi_{i}^{-1}(U) \rightarrow U$ is finite for every $i$. Then there exists a proper birational morphism $X^{\prime} \rightarrow X$ which is an isomorphism over $U$, such that the closure of $\pi_{i}^{-1}(U)$ in $Z_{i} \times_{X} X^{\prime}$ is finite over $X^{\prime}$ for every $i$.

Proof. By induction, we reduce to $n=1$; then this follows from [RG71, Corollary 5.7.10] applied with $(S, X, U) \equiv\left(X, Z_{1}, U\right)$ and $n=0$ (note that a morphism is finite if and only if it is quasi-finite and proper, and that an admissible blow-up of an algebraic space is a scheme if the algebraic space happens to be a scheme).

Theorem 1.6.2. Let $X, Y \in \mathrm{Sch}$. Let $U$ be a normal dense open subscheme of $X$, and let $\alpha$ be a finite correspondence from $U$ to $Y$. Suppose that the closure $\bar{Z}$ of $Z$ in $X \times Y$ is proper over $X$ for any component $Z$ of $\alpha$. Then there is a proper birational morphism $X^{\prime} \rightarrow X$ which is an isomorphism over $U$, such that $\alpha$ extends to a finite correspondence from $X^{\prime}$ to $Y$.

Proof. Apply Lemma 1.6.1, noting that $Z=\bar{Z} \times_{X} U$ by [KSY16, Lemma 2.6.3].

The following lemma also relies on [RG71]: it will be used several times in the sequel.

Lemma 1.6.3. Let $f: U \rightarrow X$ be an étale morphism of quasi-compact and quasi-separated integral schemes. Let $g: V \rightarrow U$ be a proper birational morphism, $T \subset U$ a closed subset such that $g$ is an isomorphism over $U-T$ and $S$ the closure of $f(T)$ in $X$. Then there exists a closed subscheme $Z \subset X$ supported in $S$ such that $U \times_{X} \mathbf{B l}_{Z}(X) \rightarrow U$ factors through $V$.

Proof. The following argument is taken from the proof of [SV00, Proposition 5.9]. Noting $V$ is étale over $X-S$, we apply the platification theorem [RG71, Corollary 5.7.11] to $V \rightarrow X$ and conclude that there exists a closed subscheme $Z$ supported in $S$ such that the proper transform $V^{\prime}$ of $V$ under $X^{\prime}=\mathbf{B l}_{Z}(X) \rightarrow X$ is flat over $X^{\prime}$. By the construction the induced morphism $\varphi: V^{\prime} \rightarrow U \times_{X} X^{\prime}$ is proper birational. On the other hand $\varphi$ is flat since it becomes flat when composed with the étale morphism $U \times_{X} X^{\prime} \rightarrow X^{\prime}$ ([Har77, Chapter II, Proposition 8.11 and Chapter III, Exercise 10.3]). Hence it is an isomorphism. This proves the lemma since $V^{\prime} \rightarrow U$ factors $V \rightarrow U$.

\subsection{Proof of Theorem 1.5.2: case of $\omega$ and $\omega_{s}$}

We need a definition:

Definition 1.7.1. Let $\sum$ be the class of all morphisms $M_{1} \rightarrow M_{2}$ in MCor given by the graph of an isomorphism $M_{1}^{\mathrm{o}} \stackrel{\sim}{\rightarrow} M_{2}^{\mathrm{o}}$ in $\mathrm{Sm}$.

In view of Proposition A.6.2, the existence of the pro-left adjoint of $\omega$ is a consequence of the following more precise result:

\section{Proposition 1.7.2.}

a) The class $\sum$ enjoys a calculus of right fractions.

b) The functor $\omega$ induces equivalences of categories

$$
\Sigma^{-1} \text { MCor } \stackrel{\sim}{\rightarrow} \text { Cor . }
$$

The same statement holds for $\omega_{s}: \mathrm{MSm} \rightarrow \mathrm{Sm}$.

Proof. a) We check the axioms of Definition A.5.1: 
(1) Identities, stability under composition: obvious.

(2) Given a diagram in MCor

$$
M_{1} \stackrel{\alpha}{ } \stackrel{M_{2}^{\prime}}{\longrightarrow} M_{2}
$$

with $M_{2}^{\mathrm{o}} \cong M_{2}^{\prime o}$, Lemma 1.1.3 provides a $M_{1}^{\prime \prime} \in$ MCor such that $M_{1}^{\prime \prime \mathrm{o}}=M_{1}^{\mathrm{o}}$ and $\alpha \in \operatorname{MCor}\left(M_{1}^{\prime \prime}, M_{2}^{\prime}\right)$. We may choose $M_{1}^{\prime \prime}$ such that $\overline{M_{1}^{\prime \prime}}=\overline{M_{1}}$. Then $M_{1}^{\prime}=\left(\overline{M_{1}}, M_{1}^{\prime \infty}\right)$ with any $M_{1}^{\prime \infty}$ such that $M_{1}^{\prime \infty} \geq$ $M_{1}^{\infty}, M_{1}^{\prime \infty} \geq M_{1}^{\prime \prime \infty}$ allows us to complete the square in MCor.

(3) Given a diagram

$$
M_{1} \underset{g}{\stackrel{f}{\rightrightarrows}} M_{2} \stackrel{s}{\rightarrow} M_{2}^{\prime}
$$

with $M_{1}, M_{2}, M_{2}^{\prime}$ as in (2) and such that $s f=s g$, the underlying correspondences to $f$ and $g$ are equal since the one underlying $s$ is $1_{M_{2}^{\mathrm{o}}}$. Hence $f=g$.

The above proof of (2) also shows that we have

$$
\underset{M^{\prime} \in \Sigma \downarrow M}{\lim _{\longrightarrow}} \operatorname{MCor}\left(M^{\prime}, N\right)=\operatorname{Cor}(M, N) .
$$

for any $M, N \in$ MCor.

Point b) now follows from a) and Corollary A.5.5, noting that $\omega$ is essentially surjective. Indeed, any smooth $k$-scheme $X$ admits a compactification $\bar{X}$ by Nagata's theorem; blowing up $\bar{X}-X$, we then make it a Cartier divisor. The case of $\omega_{s}$ is exactly parallel.

Let $\omega^{!}:$Cor $\rightarrow$ pro-MCor be the pro-left adjoint of $\omega$. By Proposition A.6.2, we have for $X \in$ Cor:

$$
\omega^{!} X={ }_{M \in \Sigma \downarrow} \lim ^{\prime} M
$$

and the same formula for the pro-left adjoint $\omega_{\mathcal{S}}^{!}$of $\omega_{s}$. Let us spell out the indexing set $\operatorname{MSm}(X)$ of these pro-objects, and refine them:

\section{Definition 1.7.3.}

(1) For $X \in \mathbf{S m}$, we define a subcategory $\mathbf{M S m}(X)$ of $\mathbf{M S m}$ as follows. The objects are those $M \in \mathbf{M S m}$ such that $M^{\mathrm{o}}=X$. Given $M_{1}, M_{2} \in \mathbf{M S m}(X)$, we define $\operatorname{MSm}(X)\left(M_{1}, M_{2}\right)$ to be $\left\{1_{X}\right\}$ if $1_{X}$ belongs to MSm and $\emptyset$ otherwise.

(2) Let $X \in \mathbf{S m}$ and fix a compactification $\bar{X}$ such that $\bar{X}-X$ is the support of a Cartier divisor (for short, a Cartier compactification). Define $\operatorname{MSm}(\bar{X} ! X)$ to be the full subcategory of $\operatorname{MSm}(X)$ consisting of objects $M \in \operatorname{MSm}(X)$ such that $\bar{M}=\bar{X}$.

Lemma 1.7.4. a) For any $X \in \mathrm{Sm}$ and any Cartier compactification $\bar{X}, \operatorname{MSm}(X)$ is a cofltered ordered set, and $\operatorname{MSm}(\bar{X} ! X)$ is cofinal in $\operatorname{MSm}(X)$.

b) Let $X \in \mathbf{C o r}$, and let $M \in \operatorname{MSm}(X)$. Then $\left\{M^{(n)}\right\}_{n \geq 1}$ defines a cofinal subcategory of $\operatorname{MSm}(X)$.

Proof. a) "Ordered" is obvious and "cofiltered" follows from Propositions 1.7.2 and A.5.2 a); the cofinality follows again from Lemma 1.1.3.

b) Let $M=\left(\bar{X}, X^{\infty}\right)$. By a) it suffices to show that $\left(M^{(n)}\right)_{n \geq 1}$ defines a cofinal subcategory of $\operatorname{MSm}(\bar{X} ! X)$. If $(\bar{X}, Y) \in \mathbf{M S m}(\bar{X} ! X), Y$ and $X^{\infty}$ both have support $\bar{X}-X$, so there exists $n>0$ such that $n X^{\infty} \geq Y$. 


\subsection{Proof of Theorem 1.5.2: case of $\tau$}

We need a definition:

Definition 1.8.1. Take $M=\left(\bar{M}, M^{\infty}\right) \in \underline{\mathbf{M S}}$ m. Let $\operatorname{Comp}(M)$ be the category whose objects are pairs $(N, j)$ consisting of a modulus pair $N=\left(\bar{N}, N^{\infty}\right) \in$ MSm equipped with a dense open immersion $j: \bar{M} \hookrightarrow \bar{N}$ such that $N^{\infty}=M_{N}^{\infty}+C$ for some effective Cartier divisors $M_{N}^{\infty}, C$ on $\bar{N}$ satisfying $\bar{N} \backslash|C|=j(\bar{M})$ and $j$ induces a minimal morphism $M \rightarrow N$ in the sense of Def. 1.3.4. Note that for $N \in \operatorname{Comp}(M)$ we have $j\left(M^{\mathrm{o}}\right)=N^{\mathrm{o}}$ and $N$ is equipped with $j_{N} \in \underline{\operatorname{MSm}}^{\text {fin }}(M, N) \subset \underline{\operatorname{MS}} \mathbf{m}(M, N)$ which is the graph of $\left.j\right|_{M^{\circ}}: M^{\mathrm{o}} \cong N^{\mathrm{o}}$. For $N_{1}, N_{2} \in \operatorname{Comp}(M)$ we define

$$
\operatorname{Comp}(M)\left(N_{1}, N_{2}\right)=\left\{\gamma \in \operatorname{MSm}\left(N_{1}, N_{2}\right) \mid \gamma \circ j_{N_{1}}=j_{N_{2}}\right\} .
$$

Note that any $\gamma$ as above induces an isomorphism $N_{1}^{\mathrm{o}} \stackrel{\sim}{\rightarrow} N_{2}^{\mathrm{o}}$ in $\mathbf{S m}$.

Lemma 1.8.2. The category $\operatorname{Comp}(M)$ is a cofiltered ordered set.

Proof. That it is ordered is obvious as $\operatorname{Comp}(M)\left(N_{1}, N_{2}\right)$ has at most 1 element for any $\left(N_{1}, N_{2}\right)$. For "cofiltered", we first show that $\operatorname{Comp}(M)$ is nonempty. For this, choose a compactification $j_{0}: \bar{M} \hookrightarrow \bar{N}_{0}$,

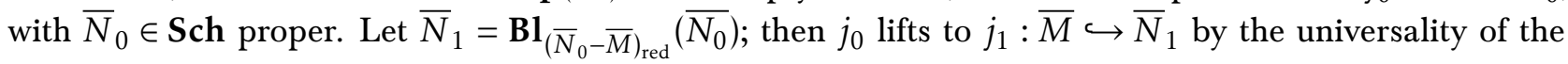
blowup [Har77, Chapter II, Proposition 7.14], and $\bar{N}_{1}-\bar{M}$ is the support of an effective Cartier divisor $C_{1}$. Consider now the scheme-theoretic closure $N_{1}^{\infty}$ of $M^{\infty}$ in $\bar{N}_{1}$, and define $\bar{N}=\mathbf{B l}_{N_{1}^{\infty}}\left(\bar{N}_{1}\right), M_{N}^{\infty}=$ pull-back of $N_{1}^{\infty}, C=$ pull-back of $C_{1}, N^{\infty}=M_{N}^{\infty}+C$ and $N=\left(\bar{N}, N^{\infty}\right)$ : then $j_{1}$ lifts to $j: \bar{M} \hookrightarrow \bar{N}$ (by the same reason as $j_{0}$ ), which defines an object of $\operatorname{Comp}(M)$.

Let $N_{1}$ and $N_{2}$ be two objects in $\operatorname{Comp}(M)$. Let $\Gamma$ be the graph of the rational map $\bar{N}_{1} \rightarrow \bar{N}_{2}$ given by $1_{M^{\circ}}$. Then we have morphisms of schemes $p: \Gamma \rightarrow \bar{N}_{1}$ and $q: \Gamma \rightarrow \bar{N}_{2}$, and there exists a natural open immersion $\bar{M} \rightarrow \Gamma$. Note that $\left(\Gamma, p^{*} N_{1}^{\infty}\right)$ and $\left(\Gamma, q^{*} N_{2}^{\infty}\right)$ are objects of $\operatorname{Comp}(M)$. Since $\left(\Gamma, p^{*} N_{1}^{\infty}\right)$ dominates $N_{1}$ and $\left(\Gamma, q^{*} N_{2}^{\infty}\right)$ dominates $N_{2}$, we are reduced to the case that $N_{1}$ and $N_{2}$ have the same ambient space $\bar{N}$. Let $C$ be the effective Cartier divisor on $\bar{N}$ such that $|C|=\bar{N}-\bar{M}$, which exists since $N_{1} \in \operatorname{Comp}(M)$. Then for a sufficiently large $n$ we have $N_{1}^{\infty}+n C \geq N_{2}^{\infty}$ since $N_{1}^{\infty} \cap \bar{M}=N_{2}^{\infty} \cap \bar{M}=M^{\infty}$. Therefore $N_{3}=\left(\bar{N}, N_{1}^{\infty}+n C\right)$ dominates both $N_{1}$ and $N_{2}$. This finishes the proof.

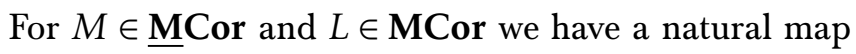

$$
\Phi: \underset{N \in \operatorname{Comp}(M)}{\lim _{\longrightarrow}} \operatorname{MCor}(N, L) \rightarrow \underline{\operatorname{MCor}(M, \tau L),}
$$

which maps a representative $\alpha_{N} \in \operatorname{MCor}(N, L)$ to $\alpha_{N} \circ j_{N}$. We also have a natural map for $M, L^{\prime} \in \underline{\text { MCor }}$

$$
\Psi: \underline{\operatorname{MCor}}\left(L^{\prime}, M\right) \rightarrow \underset{N \in \lim _{(M)}}{\operatorname{M}} \underline{\operatorname{Cor}}\left(L^{\prime}, \tau N\right)
$$

which maps $\beta$ to $\left\{j_{N} \circ \beta\right\}_{N}$.

The following is an analogue to Lemma 1.1.3:

Lemma 1.8.3. The maps $\Phi$ and $\Psi$ are isomorphisms. In other words, the formula

$$
\tau ! M=“ \lim _{N \in \operatorname{Com} \mathbf{p}(M)} ”
$$

defines a pro-left adjoint to $\tau$, which is fully faithful.

Proof. We start with $\Phi$. Injectivity is obvious since both sides are subgroups of $\operatorname{Cor}\left(M^{\mathrm{o}}, L^{\mathrm{o}}\right)$. We prove surjectivity. Choose a dense open immersion $j_{1}: \bar{M} \hookrightarrow \bar{N}_{1}$ with $\bar{N}_{1}$ proper such that $\bar{N}_{1}-\bar{M}$ is the support of an effective Cartier divisor $C_{1}$. Let $M_{1}^{\infty}$ be the scheme-theoretic closure of $M^{\infty}$ in $\bar{N}_{1}$. (This may not be Cartier.) Let $\pi: \bar{N}_{2} \rightarrow \bar{N}_{1}$ be the blowup with center in $M_{1}^{\infty}$ and put $M_{2}^{\infty}=M_{1}^{\infty} \times \bar{N}_{1} \bar{N}_{2}$ and 
$C_{2}=C_{1} \times \bar{N}_{1} \bar{N}_{2}$. Note that $M_{2}^{\infty}$ and $C_{2}$ are effective Cartier divisors on $\bar{N}_{2}$. By the universal property of the blowup [Har77, Chapter II, Proposition 7.14], $j_{1}$ extends to an open immersion $j_{2}: \bar{M} \rightarrow \bar{N}_{2}$ so that $j_{1}=\pi j_{2}$. Then $\bar{N}_{2}-M^{\mathrm{o}}$ is the support of the Cartier divisor $N_{2}^{\infty}:=M_{2}^{\infty}+C_{2}$ so that

$$
\left(\left(\bar{N}_{2}, N_{2}^{\infty}\right), j_{2}\right) \in \operatorname{Comp}(M) \text {. }
$$

Now the claim for $\Phi$ follows from the following:

Claim 1.8.4. For any $\alpha \in \underline{\operatorname{MC}} \operatorname{Cor}(M, L)$, there exists an integer $n>0$ such that $\alpha \in \operatorname{MCor}\left(\left(\bar{N}_{2}, M_{2}^{\infty}+n C_{2}\right), L\right)$.

Indeed we may assume $\alpha$ is an integral closed subscheme of $M^{\mathrm{o}} \times L^{\mathrm{o}}$. We have a commutative diagram

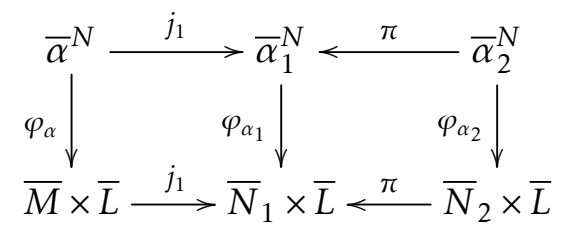

where $\bar{\alpha}^{N}$ (resp. $\bar{\alpha}_{1}^{N}$, resp. $\bar{\alpha}_{2}^{N}$ ) is the normalization of the closure of $\alpha \subset M^{\mathrm{o}} \times L^{0}$ in $\bar{M} \times \bar{L}$ (resp. $\bar{N}_{1} \times \bar{L}$, resp. $\bar{N}_{2} \times \bar{L}$ ), and $j_{1}$ and $\pi$ are induced by $j_{1}: \bar{M} \rightarrow \bar{N}_{1}$ and $\pi: \bar{N}_{2} \rightarrow \bar{N}_{1}$ respectively. Now the admissibility of $\alpha \in \underline{\operatorname{MCor}}(M, L)$ implies

$$
\varphi_{\alpha}^{*}\left(\bar{M} \times L^{\infty}\right) \leq \varphi_{\alpha}^{*}\left(M^{\infty} \times \bar{L}\right) .
$$

Since $\bar{\alpha}_{1}^{N}-j_{1}\left(\bar{\alpha}^{N}\right)$ is supported on $\varphi_{\alpha_{1}}^{-1}\left(C_{1} \times \bar{L}\right)$, this yields an inclusion of closed subschemes

$$
\varphi_{\alpha_{1}}^{*}\left(\bar{N}_{1} \times L^{\infty}\right) \subseteq \varphi_{\alpha_{1}}^{*}\left(\left(M_{1}^{\infty}+n C_{1}\right) \times \bar{L}\right)
$$

for a sufficiently large $n>0$. Applying $\pi^{*}$ to this inclusion, we get an inequality of Cartier divisors

$$
\varphi_{\alpha_{2}}^{*}\left(\bar{N}_{2} \times L^{\infty}\right) \leq \varphi_{\alpha_{2}}^{*}\left(\left(M_{2}^{\infty}+n C_{2}\right) \times \bar{L}\right)
$$

which proves the claim.

Next we prove that $\Psi$ is an isomorphism. Injectivity is obvious since both sides are subgroups of $\operatorname{Cor}\left(L^{\mathrm{o}}, M^{\mathrm{o}}\right)$. We prove surjectivity. Take

$$
\gamma \in \lim _{N \in \operatorname{Comp}(M)} \operatorname{MCor}(L, N)
$$

Then $\gamma \in \operatorname{Cor}\left(L^{\mathrm{o}}, M^{\mathrm{o}}\right)$ is such that any component $\delta \subset L^{\mathrm{o}} \times M^{\mathrm{o}}$ of $\gamma$ satisfies the following condition: take any $(N, j) \in \operatorname{Comp}(M)$ and write $N^{\infty}=M_{N}^{\infty}+C$ as in Definition 1.8.1. Let $\bar{\delta}^{N}$ be the normalization of the closure of $\delta$ in $\bar{L} \times \bar{N}$ with the natural map $\varphi_{\delta}: \bar{\delta}^{N} \rightarrow \bar{L} \times \bar{N}$. Then we have

$$
\varphi_{\delta}^{*}\left(\bar{L} \times\left(M_{N}^{\infty}+n C\right)\right) \leq \varphi_{\delta}^{*}\left(L^{\infty} \times \bar{N}\right)
$$

for any integer $n>0$. Clearly this implies that $|\bar{\delta}|$ does not intersect with $\bar{L} \times|C|$ so that $\bar{\delta} \subset \bar{L} \times \bar{M}$. Since $\bar{\delta}$ is proper over $\bar{L}$ by assumption, this implies $\delta \in \underline{\operatorname{MCor}}(L, M)$ which proves the surjectivity of $\Psi$ as desired.

We come back to the proof of Theorem 1.5.2. It remains to consider $\tau_{s}$. The natural maps

$$
\begin{aligned}
& \varphi: \underset{N \in \underset{\operatorname{Comp}}{\longrightarrow}(M)}{\lim } \operatorname{MSm}(N, L) \rightarrow \underline{\operatorname{MSm}}(M, \tau L),
\end{aligned}
$$

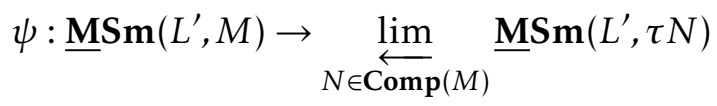

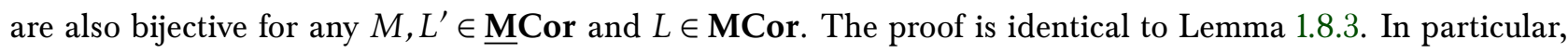
the inclusion functor $\tau_{s}: \mathbf{M S m} \rightarrow \underline{\mathbf{M S m}}$ admits a pro-left adjoint given by

$$
\tau_{s}^{!} M=“ \underset{N \in \operatorname{Com} \mathbf{p}(M)}{\lim ^{\prime}} N
$$




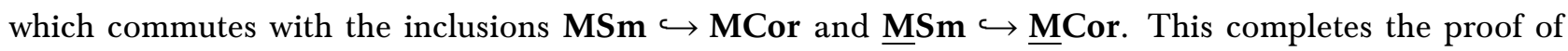
Theorem 1.5.2.

\subsection{More on $\underline{M S m}^{\text {fin }}$ and $\underline{\mathrm{MC}}{ }^{\mathrm{fin}}$}

Definition 1.9.1. A morphism $f: M \rightarrow N$ in $\underline{\mathbf{M S m}}^{\text {fin }}$ is in $\underline{\Sigma}^{\text {fin }}$ if it is minimal (Definition 1.3.4), $\bar{f}: \bar{M} \rightarrow \bar{N}$ is a proper morphism and $f^{\mathrm{o}}$ is an isomorphism in $\mathrm{Sm}$. We write $\Sigma^{\text {fin }}$ for the class of morphisms in $\underline{\Sigma}^{\text {fin }}$ that belong to MSm.

In particular, we have $\Sigma^{\text {fin }} \subset \sum$ (see Definition 1.7.1) and $\underline{\Sigma}^{\text {fin }} \downarrow M=\Sigma^{\text {fin }} \downarrow M$ for $M \in$ MSm. Let us consider the inclusion functors

$$
\underline{b}_{s}: \underline{\mathbf{M S m}}^{\mathrm{fin}} \rightarrow \underline{\mathbf{M S m}}, \underline{b}: \underline{\mathbf{M}} \operatorname{Cor}^{\mathrm{fin}} \rightarrow \underline{\mathbf{M C o r}} .
$$

The following commutative diagram of categories will become fundamental (cf. (2.7.1)):

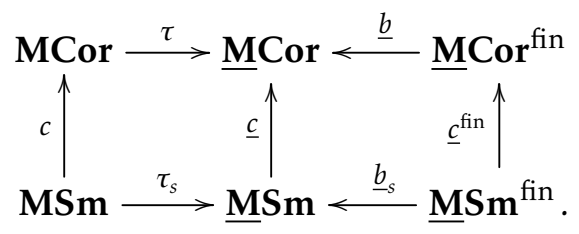

\section{Proposition 1.9.2.}

a) The class $\underline{\Sigma}^{\text {fin }}$ enjoys a calculus of right fractions within $\underline{\mathbf{M S m}}{ }^{\text {fin }}$ and $\underline{\mathbf{M C o r}}{ }^{\mathrm{fin}}$.

b) The functors $\underline{b}_{s}$ and $\underline{b}$ are localisations having left pro-adjoints $b_{s}^{!}$and $b$. They induce equivalences of categories

$$
\left(\underline{\Sigma}^{\mathrm{fin}}\right)^{-1} \underline{\mathbf{M S m}} \mathbf{m}^{\mathrm{fin}} \cong \underline{\mathbf{M S m}} \text { and }\left(\underline{\Sigma}^{\mathrm{fin}}\right)^{-1} \underline{\mathbf{M C o r}}^{\mathrm{fin}} \cong \underline{\mathbf{M}} \text { Cor } .
$$

c) A morphism in $\underline{\mathrm{MCor}}^{\mathrm{fin}}$ (resp. $\underline{\mathrm{MSm}}{ }^{\mathrm{fin}}$ ) is invertible in $\underline{\mathrm{MCor}}$ (resp. $\underline{\mathrm{MSm}}$ ) if and only if it belongs to $\underline{\Sigma}^{\mathrm{fin}}$. A morphism $f$ in $\underline{\mathrm{MCor}}$ (resp. $\underline{\mathrm{MSm}}$ ) is an isomorphism if and only if it can be written as $s=s_{1} s_{2}^{-1}$ for some $s_{1}, s_{2} \in \underline{\Sigma}^{\mathrm{fin}}$.

All statements hold for $\sum^{\text {fin }}$ (without an underline) as well.

Proof. a) Same as the proof of Proposition 1.7.2 a), except for (2): consider a diagram in $\underline{\text { MCor }}{ }^{\text {fin }}$

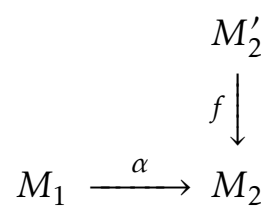

with $f \in \underline{\Sigma}^{\text {fin }}$ (in particular $f^{\mathrm{o}}$ is an isomorphism). By the properness of $f$, the finite correspondence $\alpha^{\mathrm{o}}: M_{1}^{\mathrm{o}} \rightarrow M_{2}^{\prime \mathrm{o}}$ satisfies the hypothesis of Theorem 1.6.2. Applying this theorem, we find a proper birational morphism $f^{\prime}: \bar{M}_{1}^{\prime} \rightarrow \bar{M}_{1}$ which is an isomorphism over $M_{1}^{\mathrm{o}}$ and such that $\alpha^{\mathrm{o}}$ defines a finite correspondence $\alpha^{\prime}: \bar{M}_{1}^{\prime} \rightarrow \bar{M}_{2}^{\prime}$. If we define $M_{1}^{\prime \infty}=f^{\prime *} M_{1}^{\infty}$, then $f^{\prime} \in \underline{\Sigma}^{\text {fin }}$ and $\alpha^{\prime} \in \underline{\operatorname{MCor}}{ }^{\text {fin }}\left(M_{1}^{\prime}, M_{2}^{\prime}\right)$.

If $\alpha \in \underline{\mathbf{M S m}}^{\text {fin }}\left(M_{1}, M_{2}\right)$, then $\alpha^{\prime}$ is not in $\underline{\mathbf{M S}}{ }^{\text {fin }}\left(M_{1}^{\prime}, M_{2}^{\prime}\right)$ in general (unless $\overline{M_{1}^{\prime}}$ is normal, see Remark 1.3.5 (1)). However, write $\overline{M_{1}^{\prime \prime}}$ for the closure of the graph of the rational map $\alpha^{\prime}: \overline{M_{1}^{\prime}} \rightarrow \overline{M_{2}^{\prime}}$, and $\pi$ for the projection $\overline{M_{1}^{\prime \prime}} \rightarrow \overline{M_{1}^{\prime}}$ : by hypothesis, $\pi$ is finite birational. Define a modulus pair $M_{1}^{\prime \prime}=\left(\overline{M_{1}^{\prime \prime}}, M_{1}^{\prime \prime \infty}\right)$ by putting $M_{1}^{\prime \prime \infty}:=\pi^{*} M_{1}^{\prime \infty}$. Then $\pi$ defines a minimal morphism $M_{1}^{\prime \prime} \rightarrow M_{1}^{\prime}$ in $\underline{\mathbf{M S}} \mathbf{m}^{\text {fin }}$, hence the morphism $\alpha^{\prime \prime}: M_{1}^{\prime \prime} \rightarrow M_{2}^{\prime}$ determined by $\alpha^{\prime}$ is in $\underline{\mathbf{M S}} \mathbf{m}^{\text {fin }}$.

For b), all assertions are obvious except for the equivalences, for which it suffices as in Corollary A.5.5 to show that for any $M, N \in \underline{\text { MCor, the obvious maps }}$

$$
\underset{M^{\prime} \in \underline{\Sigma}^{\mathrm{fin}} \downarrow M}{\lim } \underline{\operatorname{MCor}} \operatorname{Con}^{\operatorname{fin}}\left(M^{\prime}, N\right) \rightarrow \underline{\operatorname{MCor}}(M, N)
$$


and the corresponding map for $\underline{b}_{s}$ are isomorphisms. These maps are clearly injective, and its surjectivity follows again from Theorem 1.6.2. It then follows from Proposition A.6.2 they have pro-left adjoints.

The first statement of c) is clear, and the second follows from b).

The same proof works for $\Sigma^{\text {fin }}$.

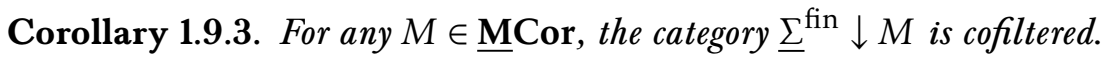

Proof. This follows from Propositions 1.9.2 and A.5.2.

Corollary 1.9.4. Let $\mathcal{C}$ be a category and let $F: \underline{\operatorname{MCor}}{ }^{\text {fin }} \rightarrow \mathcal{C}, G: \underline{\mathbf{M S m}} \rightarrow \mathcal{C}$ be two functors whose restrictions to the common subcategory $\underline{\mathbf{M S}}{ }^{\text {fin }}$ are equal. Then $(F, G)$ extends (uniquely) to a functor $H: \underline{\mathbf{M} C o r} \rightarrow \mathcal{C}$.

Proof. The hypothesis implies that $F$ inverts the morphisms in $\underline{\Sigma}^{\text {fin }}$; the conclusion now follows from Proposition 1.9.2 b).

Corollary 1.9.5. Any modulus pair in $\underline{\mathrm{MSm}}$ is isomorphic to a modulus pair $M$ in which $\bar{M}$ is normal. Under resolution of singularities, we may even choose $\bar{M}$ smooth and the support of $M^{\infty}$ to be a divisor with normal crossings.

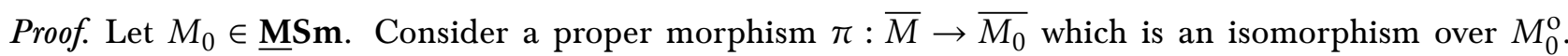
Define $M^{\infty}:=\pi^{*} M_{0}^{\infty}$. Then the induced morphism $\pi: M \rightarrow M_{0}$ of $\underline{\mathbf{M S m}}^{\text {fin }}$ is in $\underline{\Sigma}^{\mathrm{fin}}$, hence invertible in MSm. The corollary readily follows.

We also have the following important lemma:

Lemma 1.9.6. Let $M, L, N \in \underline{\mathbf{M S m}}$. Let $f: L \rightarrow N$ be a minimal morphism in $\underline{\mathbf{M S m}}{ }^{\text {fin }}$ such that $\bar{f}: \bar{L} \rightarrow \bar{N}$ is faithfully flat. Then the diagram

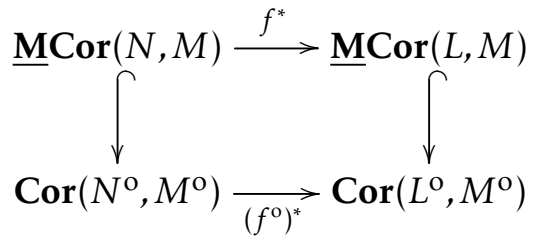

is cartesian. The same holds when $\underline{\mathrm{MC}}$ or is replaced by $\underline{\mathrm{MCor}^{\mathrm{fin}}}$.

Proof. As the second statement is proven in a completely parallel way, we only prove the first one. Take $\alpha \in \operatorname{Cor}\left(N^{\circ}, M^{o}\right)$ such that $\left(f^{\circ}\right)^{*}(\alpha) \in \underline{\operatorname{MCor}}(L, M)$. We need to show $\alpha \in \underline{\operatorname{MCor}}(N, M)$.

We first reduce to the case where $\alpha$ is integral. To do this, it suffices to show that for two distinct integral finite correspondences $V, V^{\prime} \in \operatorname{Cor}\left(N^{\mathrm{o}}, M^{\mathrm{o}}\right),\left(f^{\mathrm{o}}\right)^{*}(V)$ and $\left(f^{\mathrm{o}}\right)^{*}\left(V^{\prime}\right)$ have no common component. For this, we may assume $M^{\mathrm{o}}$ and $N^{\mathrm{o}}$ integral. By the injectivity of $\operatorname{Cor}\left(N^{\mathrm{o}}, M^{\mathrm{o}}\right) \rightarrow \operatorname{Cor}\left(k\left(N^{\mathrm{o}}\right), M^{\mathrm{o}}\right)$, this can be reduced to the case where $N^{\mathrm{o}}$ and $L^{\mathrm{O}}$ are fields, and then the claim is obvious.

Now assume $\alpha$ is integral and put $\beta:=\left(f^{\circ}\right)^{*}(\alpha)$. We have a commutative diagram

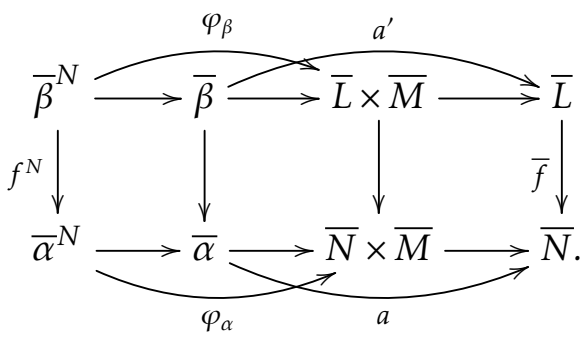

Here $\bar{\alpha}$ (resp. $\bar{\beta})$ is the closure of $\alpha$ (resp. $\beta$ ) in $\bar{N} \times \bar{M}\left(\right.$ resp. $\bar{L} \times \bar{M}$ ) and $\bar{\alpha}^{N}\left(\right.$ resp. $\bar{\beta}^{N}$ ) is the normalization of $\bar{\alpha}$ (resp. $\bar{\beta}$ ). By hypothesis $a^{\prime}$ is proper and $\bar{f}$ is faithfully flat. This implies that $a$ is proper [SGA1, 
exposé VIII, corollaire 4.8]. We also have

$$
\begin{aligned}
\left(f^{N}\right)^{*}\left(\varphi_{\alpha}^{*}\left(N^{\infty} \times \bar{M}\right)\right) & \left.=\varphi_{\beta}^{*}\left(\bar{f}^{*}\left(N^{\infty}\right) \times \bar{M}\right)\right) \\
=\varphi_{\beta}^{*}\left(L^{\infty} \times \bar{M}\right) & \geq \varphi_{\beta}^{*}\left(\bar{L} \times M^{\infty}\right)=\left(f^{N}\right)^{*}\left(\varphi_{\alpha}^{*}\left(\bar{N} \times M^{\infty}\right)\right)
\end{aligned}
$$

(the second equality by the minimality of $f$ ). Note that $f^{N}$ is surjective since $\bar{f}$ is. Hence Lemma 1.2.1 shows that $\varphi_{\alpha}^{*}\left(N^{\infty} \times \bar{M}\right) \geq \varphi_{\alpha}^{*}\left(\bar{N} \times M^{\infty}\right)$, and we are done.

\subsection{Fiber products and squarable morphisms}

We need the following elementary lemma.

Lemma 1.10.1. Let $X$ be a scheme. For two effective Cartier divisors $D$ and $E$ on $X$, the following conditions are equivalent:

(1) $D \times_{X} E$ is an effective Cartier divisor on $X$.

(2) There exist effective Cartier divisors $D^{\prime}, E^{\prime}$ and $F$ on $X$ such that $D=D^{\prime}+F, E=E^{\prime}+F$ and $\left|D^{\prime}\right| \cap\left|E^{\prime}\right|=\emptyset$. Moreover, the divisors $D^{\prime}, E^{\prime}$ and $F$ satisfying the conditions in (2) are uniquely determined by $D$ and $E$.

Proof. We may suppose $X=\operatorname{Spec} A$ is affine and $D, E$ are defined by non-zero-divisors $d, e \in A$, respectively.

Suppose (1). This means that $(d, e)=(f)$ for some non-zero-divisor $f \in A$, because $D \times_{X} E=\operatorname{Spec} A /(d, e)$. Thus there are $d^{\prime}, e^{\prime} \in A$ such that $d=d^{\prime} f$ and $e=e^{\prime} f$. Since $(f)=\left(d^{\prime}, e^{\prime}\right)(f)$ and $f$ is a non-zero-divisor, we have $\left(d^{\prime}, e^{\prime}\right)=A$. Now (2) holds by taking $D^{\prime}, E^{\prime}, F$ to be the Cartier divisors defined by $\left(d^{\prime}\right),\left(e^{\prime}\right)$ and $(f)$.

(2) immediately implies $F=D \times_{X} E$, whence (1). This formula also implies the uniqueness of $F$, hence $D^{\prime}=D-F$ and $E^{\prime}=E-F$ are unique as well.

Definition 1.10.2. Let $D$ and $E$ be effective Cartier divisors on a scheme $X$. If the conditions of Lemma 1.10.1 hold, we say that $D$ and $E$ have a universal supremum, and write

$$
\sup (D, E):=D^{\prime}+E^{\prime}+F(=D+E-F) .
$$

Remark 1.10.3. Let $D$ and $E$ be effective Cartier divisors on $X$ having a universal supremum. The following are obvious from the definition.

(1) We have $|\sup (D, E)|=|D| \cup|E|$.

(2) If $f: Y \rightarrow X$ is a morphism such that $f(T) \not \subset|D| \cup|E|$ for any irreducible component $T$ of $Y$, then $f^{*} D$ and $f^{*} E$ have a universal supremum which is equal to $f^{*} \sup (D, E)$ (hence the name "universal").

(3) If moreover $Y$ is normal, then $f^{*} \sup (D, E)$ agrees with the supremum of $f^{*} D$ and $f^{*} E$ computed as a Weil divisor on $Y$.

Let $u_{i}: U_{i} \rightarrow M$ be morphisms in $\underline{\mathbf{M S}} \mathbf{m}^{\text {fin }}$ for $i=1,2$ with projections $p_{i}: \bar{W}_{0}:=\bar{U}_{1} \times \bar{M}_{2} \bar{U}_{2} \rightarrow \bar{U}_{i}$. Denote by $\bar{W}_{1}$ the union of irreducible components $T$ of $\bar{W}_{0}$ such that $p_{i}(T) \not \subset\left|U_{i}^{\infty}\right|$ for each $i=1,2$. Observe that $\bar{W}_{1}$ is the closure of $U:=U_{1}^{\mathrm{o}} \times_{M^{\circ}} U_{2}^{\mathrm{o}}$ in $\bar{W}_{0}$. Indeed, let $Z$ be the closure of $U$ in $\bar{W}_{0}$. Then any irreducible component $T$ of $Z$ meets $U$, which implies that $T \subset \bar{W}_{1}$. Conversely, any irreducible component $T$ of $\bar{W}_{1}$ meets $U$, hence $T \cap U$ is dense in $T$ and thus $T \subset Z$.

We write $q_{i}: \bar{W}_{1} \rightarrow \bar{U}_{i}$ for the composition of the inclusion $\bar{W}_{1} \rightarrow \bar{W}_{0}$ and $p_{i}$. By definition, we have effective Cartier divisors $q_{i}^{*}\left(U_{i}^{\infty}\right)$ on $\bar{W}_{1}$ and $q_{1} \times q_{2}$ restricts to an isomorphism

$$
\bar{W}_{1} \backslash\left|q_{1}^{*}\left(U_{1}^{\infty}\right)+q_{2}^{*}\left(U_{2}^{\infty}\right)\right| \simeq U_{1}^{\mathrm{o}} \times_{M^{\circ}} U_{2}^{\mathrm{o}} .
$$

Proposition 1.10.4. Suppose that $U_{1}^{\mathrm{o}} \times_{M^{\circ}} U_{2}^{\mathrm{o}}$ is smooth over $k$.

(1) If $q_{1}^{*} U_{1}^{\infty}$ and $q_{2}^{*} U_{2}^{\infty}$ have a universal supremum, then

$$
W_{1}:=\left(\bar{W}_{1}, \sup \left(q_{1}^{*} U_{1}^{\infty}, q_{2}^{*} U_{2}^{\infty}\right)\right) \in \underline{\mathbf{M S m}}^{\text {fin }}
$$


represents the fiber product of $U_{1}$ and $U_{2}$ over $M$ in $\underline{\mathbf{M S m}}^{\text {fin }}$ as well as in $\underline{\mathbf{M S m}}$. If further $U_{1}, U_{2}, M \in$ $\mathbf{M S m}^{\text {fin }}$, then it holds in $\mathbf{M S m}^{\text {fin }}$ as well as in $\mathbf{M S m}$.

(2) If $u_{1}$ is minimal and $\bar{U}_{2}$ is normal, then $q_{1}^{*} U_{1}^{\infty}$ and $q_{2}^{*} U_{2}^{\infty}$ have a universal supremum, namely $q_{2}^{*} U_{2}^{\infty}$,

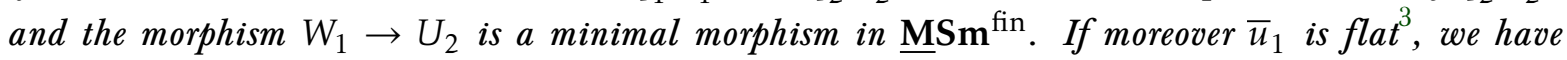
$\bar{W}_{1}=\bar{W}_{0}$.

(3) In general, there is a proper birational morphism $\pi: \bar{W}_{2} \rightarrow \bar{W}_{1}$ which restricts to an isomorphism over $\bar{W}_{1} \backslash\left|q_{1}^{*}\left(U_{1}^{\infty}\right)+q_{2}^{*}\left(U_{2}^{\infty}\right)\right|$, and such that $r_{1}^{*} U_{1}^{\infty}$ and $r_{2}^{*} U_{2}^{\infty}$ have a universal supremum, where $r_{i}:=q_{i} \pi$ for $i=1,2$. For such $\bar{W}_{2}$,

$$
W_{2}:=\left(\bar{W}_{2}, \sup \left(r_{1}^{*} U_{1}^{\infty}, r_{2}^{*} U_{2}^{\infty}\right)\right) \in \underline{\mathbf{M S m}}
$$

represents the fiber product of $U_{1}$ and $U_{2}$ over $M$ in $\underline{\mathbf{M S m}}$. If further $U_{1}, U_{2}, M \in \mathbf{M S m}$, then it holds in MSm.

Proof. (1) Let $f_{i}: N \rightarrow U_{i}$ be morphisms in $\underline{M S m}^{\text {fin }}$ for $i=1,2$ such that $u_{1} f_{1}=u_{2} f_{2}$. Then the morphisms $\bar{f}_{i}: \bar{N} \rightarrow \bar{U}_{i}$ for $i=1,2$ induce a unique morphism $\bar{h}: \bar{N} \rightarrow \bar{W}_{0}$ with $\bar{f}_{i}=p_{i} \bar{h}$ for $i=1,2$. Since $f_{i}$ are morphisms in $\underline{\mathbf{M S m}}{ }^{\text {fin }}$, for any irreducible component $T$ of $\bar{N}$ we have $\bar{f}_{i}(T) \not \subset\left|U_{i}^{\infty}\right|$, and hence $\bar{h}$ factors though $\bar{g}: \bar{N} \rightarrow \bar{W}_{1}$ so that we have $\bar{f}_{i}=q_{i} \bar{g}$. It remains to prove $v^{*} N^{\infty} \geq v^{*} \bar{g}^{*} W_{1}^{\infty}$, where $v: \bar{N}^{N} \rightarrow \bar{N}$ is the normalization. As we have $v^{*} \bar{g}^{*} W_{1}^{\infty}=v^{*} \bar{g}^{*} \sup \left(q_{1}^{*} U_{1}^{\infty}, q_{2}^{*} U_{2}^{\infty}\right)=\sup \left(v^{*} \bar{f}_{1}^{*} U_{1}^{\infty}, v^{*} \bar{f}_{1}^{*} U_{2}^{\infty}\right)$ by definition and Remark 1.10.3, this follows from the admissibility of $f_{i}$, that is, $v^{*} \bar{f}_{i}^{*} U_{i}^{\infty} \leq v^{*} N^{\infty}$. We have shown that $W_{1}$ represents the fiber product in $\underline{\mathbf{M S}} \mathbf{m}^{\text {fin }}$. Propositions 1.9.2 and A.5.6 show that the same holds in $\underline{\mathbf{M S m}}$ as well. (This also follows from (3) below.) The last statement is an immediate consequence of the first.

(2) Let $p_{W}: \bar{W}_{1}^{N} \rightarrow \bar{W}_{1}$ and $p_{U_{1}}: \bar{U}_{1}^{N} \rightarrow \bar{U}_{1}$ be the normalizations. By the minimality of $u_{1}$, we have $q_{1}^{*} U_{1}^{\infty}=q_{1}^{*} \bar{u}_{1}^{*} M^{\infty}=q_{2}^{*} \bar{u}_{2}^{*} M^{\infty} \leq q_{2}^{*} U_{2}^{\infty}$, where the last inequality holds by the admissibility of $u_{2}$ and the normality of $\bar{U}_{2}$. Then $q_{1}^{*} U_{1}^{\infty}$ and $q_{2}^{*} U_{2}^{\infty}$ have a universal supremum since $q_{1}^{*} U_{1}^{\infty} \subset q_{2}^{*} U_{2}^{\infty}$ implies Condition (1) of Lemma 1.10.1, which also implies that $W_{1}^{\infty}=\sup \left(q_{1}^{*} U_{1}^{\infty}, q_{2}^{*} U_{2}^{\infty}\right)=q_{2}^{*} U_{2}^{\infty}$. This shows the minimality of $W_{1} \rightarrow U_{2}$.

Suppose now $\bar{u}_{1}$ flat, and let $T$ be an irreducible component of $\bar{W}_{0}$. Then $p_{2}: \bar{W}_{0} \rightarrow \bar{U}_{2}$ is also flat, hence $T$ dominates an irreducible component $E$ of $\bar{U}_{2}$ [Har77, Chapter III, Proposition 9.5] and we cannot have $p_{2}(T) \subset\left|U_{2}^{\infty}\right|$ since $U_{2}^{\infty}$ is everywhere of codimension 1 in $\bar{U}_{2}$. Suppose that $p_{1}(T) \subset\left|U_{1}^{\infty}\right|$. By the minimality of $u_{1}$, this implies $u_{2} p_{2}(T)=u_{1} p_{1}(T) \subset\left|M^{\infty}\right|$, hence $u_{2}(E) \subset\left|M^{\infty}\right|$, contradicting the admissibility of $u_{2}$.

(3) If $\pi$ is the blow-up of $\bar{W}_{1}$ with center $q_{1}^{*}\left(U_{1}^{\infty}\right) \times \bar{W}_{1} q_{2}^{*}\left(U_{1}^{\infty}\right)$, then $r_{1}^{*} U_{1}^{\infty} \times \bar{W}_{2} r_{2}^{*} U_{2}^{\infty}$ is precisely the exceptional divisor by definition, which is therefore an effective Cartier divisor, showing the first assertion. Note that $W_{2}^{\mathrm{o}} \cong U_{1}^{\mathrm{o}} \times_{M^{\mathrm{o}}} U_{2}^{\mathrm{o}}$ by $(1.10 .1)$.

Now let $f_{i}: N \rightarrow U_{i}$ be morphisms in $\underline{\mathbf{M S m}}$ for $i=1,2$ such that $u_{1} f_{1}=u_{2} f_{2}$. Then the morphisms $f_{i}^{\mathrm{o}}: N^{\mathrm{o}} \rightarrow U_{i}^{\mathrm{o}}$ for $i=1,2$ induce a unique morphism $h^{\mathrm{o}}: N^{\mathrm{o}} \rightarrow W_{2}^{\mathrm{o}}$ with $f_{i}^{\mathrm{o}}=p_{i} h^{\mathrm{o}}$ for $i=1,2$. It suffices to prove that $h^{\mathrm{o}}$ defines a [unique] morphism in $\underline{\mathbf{M S m}}$. By the graph trick (Lemma 1.3.6), we may assume that $f_{i}^{\mathrm{o}}$ and $h^{\mathrm{o}}$ extend to morphisms $\bar{f}_{i}: \bar{N} \rightarrow \bar{U}_{i}$ and $\bar{h}: \bar{N} \rightarrow \bar{W}_{2}$. Moreover we may assume that $\bar{N}$ is normal by Remark 1.3.5 (2). It remains to prove $N^{\infty} \geq \bar{h}^{*} W_{2}^{\infty}$. As we have $\bar{h}^{*} W_{2}^{\infty}=\sup \left(\bar{f}_{1}^{*} U_{1}^{\infty}, \bar{f}_{1}^{*} U_{2}^{\infty}\right)$ by the assumption and Remark 1.10.3, this follows from the admissibility of $f_{i}$, that is, $\bar{f}_{i}^{*} U_{i}^{\infty} \leq N^{\infty}$.

Remark 1.10.5. If $W$ represents a fiber product $U_{1} \times_{M} U_{2}$ (either in $\underline{\mathbf{M S m}}$ or in $\underline{\mathbf{M S m}}{ }^{\text {fin }}$ ), then we have $W^{\mathrm{o}}=U_{1}^{\mathrm{o}} \times M^{\mathrm{o}} U_{2}^{\mathrm{o}}$. Indeed, the functors $\underline{\mathbf{M S m}} \rightarrow \mathbf{S m}$ and $\underline{\mathbf{M S}} \mathbf{S m}^{\text {fin }} \rightarrow \mathbf{S m}$ given by $M \mapsto M^{\mathrm{o}}$ have the left adjoint $X \mapsto(X, \emptyset)$ (Lemma 1.5.1), hence commute with limits.

Examples 1.10.6. Let $B=k\left[x_{1}, x_{2}\right], \mathbf{A}^{2}=\operatorname{Spec} B, D_{i}=\operatorname{Spec}\left(B / x_{i} B\right)$ and $P=D_{1} \cap D_{2}$. Let now $M=$ $\left(D_{1} \cup D_{2}, P\right)$ and $U_{i}=\left(D_{i}, P\right)$ for $i=1,2$. Then $\bar{W}_{0}$ is a point but $\bar{W}_{1}=\emptyset$, and $W_{1}=(\emptyset, \emptyset)$ indeed

\footnotetext{
${ }^{3} \mathrm{By}$ the local criterion of flatness [Har77, Chapter III, Lemma 10.3.A], this is equivalent to the flatness of $U_{1}^{\infty} \rightarrow M^{\infty}$.
} 
represents the fiber product $U_{1} \times_{M} U_{2}$. In particular, fiber products do not commute with the forgetful functor $M \mapsto \bar{M}$ from $\underline{M S m}^{\text {fin }}$ to Sch of Definition 1.3.3 (2). Another counterexample: let $M=\left(\mathbf{A}^{2}, D_{1}\right)$, $\bar{U}_{1}=\mathbf{B} \mathbf{l}_{P}\left(\mathbf{A}^{2}\right), u_{1}: U_{1} \rightarrow M$ be the minimal induced modulus structure, $U_{2}=\left(D_{2}, P\right)$ and $U_{2} \rightarrow M$ be given by the inclusion. Then $\bar{W}_{1} \subsetneq \bar{W}_{0}$ is the proper transform of $u_{1}$. See however Corollary 1.10.7 (1).

Recall [SGA3, exposé IV, définition 1.4.0] that a morphism $f: M \rightarrow N$ in a category $\mathcal{C}$ is squarable if, for any $g: N^{\prime} \rightarrow N$, the fibred product $N^{\prime} \times_{N} M$ is representable in $\mathcal{C}$. We have:

Corollary 1.10.7. The following assertions hold.

(1) If $f: U \rightarrow M$ is a minimal morphism in MSm $^{\text {fin }}$ (see Definition 1.3.4) such that $f^{\mathrm{o}}$ is smooth, then $f$ is squarable in $\mathbf{M S m}^{\text {fin }}$. If $f \in \mathbf{M S m}^{\text {fin }}$, it is squarable in this category. If moreover $\bar{f}$ is flat, then the pull-back by $f$ of morphisms from normal modulus pairs commutes with the forgetful functor $M \mapsto \bar{M}$ from $\underline{M S m}^{\text {fin }}$ to Sch of Definition 1.3.3 (2).

(2) If $f: U \rightarrow M$ is a morphism in $\underline{\mathrm{MS}}$ such that $f^{\circ}$ is smooth, then $f$ is squarable in $\underline{\mathrm{MSm}}$. If $f \in \mathbf{M S m}$, it is squarable in this category.

Proof. (1) follows from Proposition 1.10.4 (1) and (2); (2) follows from Proposition 1.10.4 (3).

Corollary 1.10.8. Finite products exist in $\underline{\mathrm{MSm}}$ and MSm.

Proof. This is the special case $M=(\operatorname{Spec} k, \emptyset)$ in Corollary 1.10 .7 (2).

\section{Presheaf theory}

\subsection{Modulus presheaves with transfers}

Definition 2.1.1. By a presheaf we mean a contravariant functor to the category of abelian groups.

(1) The category of presheaves on MSm (resp. $\underline{\mathbf{M S m}}, \underline{\mathbf{M S m}}^{\mathrm{fin}}$ ) is denoted by MPS (resp. $\underline{\text { MPS, }} \underline{\mathbf{M P S}}{ }^{\mathrm{fin}}$ ).

(2) The category of additive presheaves on MCor (resp. $\underline{\text { MCor, }} \underline{\text { MCor }}{ }^{f i n}$ ) is denoted by MPST (resp. MPST, MPST $^{\text {fin }}$.)

All these categories are abelian Grothendieck, with projective sets of generators: this is classical for those of (1) and follows from Theorem A.10.2 for those of (2). (See also proof of Proposition 2.6.1 below.)

Notation 2.1.2. We write

$$
\begin{aligned}
& \mathbb{Z}_{\mathrm{tr}}: \underline{\text { MCor }} \rightarrow \underline{\text { MPST }}, \quad \text { MCor } \rightarrow \text { MPST }, \\
& \mathbb{Z}_{\mathrm{tr}}^{\mathrm{fin}}: \underline{\text { MCor }}^{\mathrm{fin}} \rightarrow \underline{\text { MPST }}^{\text {fin }}, \\
& \mathbb{Z}_{\mathrm{tr}}: \text { Cor } \rightarrow \text { PST }
\end{aligned}
$$

for the associated representable presheaves (i.e. $\mathbb{Z}_{\mathrm{tr}}(M) \in \underline{\text { MPST }}$ is given by $\mathbb{Z}_{\mathrm{tr}}(M)(N)=\underline{\operatorname{MCor}}(N, M)$, etc.) We shall use the common notation $\mathbb{Z}_{\text {tr }}$ but they will be distinguished by the context.

We now briefly describe the main properties of the functors induced by those of the previous section.

\subsection{MPST and PST}

We say $\left(f_{1}, f_{2}, \ldots, f_{n}\right)$ is a string of adjoint functors if $f_{i}$ is a left adjoint of $f_{i+1}$ for each $i=1, \ldots, n-1$.

Proposition 2.2.1. The functor $\omega:$ MCor $\rightarrow$ Cor of $\$ 1.5$ yields a string of three adjoint functors $\left(\omega_{!}, \omega^{*}, \omega_{*}\right)$ :

$$
\operatorname{MPST} \underset{\underset{\omega_{*}}{\rightleftarrows}}{\stackrel{\omega_{!}}{\rightleftarrows}} \text { PST, }
$$


where $\omega^{*}$ is fully faithful and $\omega_{!}, \omega_{*}$ are localisations; $\omega_{!}$has a pro-left adjoint $\omega^{!}$, hence is exact.

Similarly, $\omega_{s}: \mathbf{M S m} \rightarrow \mathbf{S m}$ yields a string of three adjoint functors $\left(\omega_{s !}, \omega_{S^{*}}^{*}, \omega_{s^{*}}\right) ; \omega_{s}^{*}$ is fully faithful and $\omega_{s !}, \omega_{s *}$ are localisations; $\omega_{s !}$ has a pro-left adjoint $\omega_{s}^{!}$, hence is exact.

Proof. This follows from Theorems 1.7.2 and A.6.5.

Let $X \in \mathrm{Sm}$ and let $M \in \mathbf{M S m}(X)$. Lemma 1.7.4 and Proposition A.4.1 show that the inclusions $\left\{M^{(n)} \mid n>0\right\} \subset \mathbf{M S m}(\bar{M} ! X) \subset \mathbf{M S m}(X)$ induce isomorphisms (see Definition 1.7.3)

$$
\omega_{!}(F)(X) \simeq \underset{N \in \underset{\mathbf{M S m}}{\longrightarrow}(X)}{\lim } F(N) \simeq \underset{N \in \underset{\mathbf{M S m}}{\longrightarrow}(\bar{M} ! X)}{\lim } F(N) \simeq \underset{n>0}{\lim } F\left(M^{(n)}\right) .
$$

\subsection{MPST and PST}

Proposition 2.3.1. The adjoint functors $(\lambda, \underline{\omega})$ of Lemma 1.5.1 induce a string $\left(\lambda_{!}=\underline{\omega}^{!}, \lambda^{*}=\underline{\omega}, \lambda_{*}=\underline{\omega}^{*}, \underline{\omega}_{*}\right)$ of four adjoint functors:

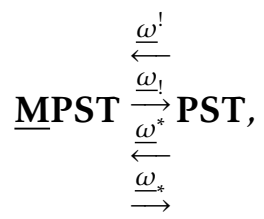

where $\underline{\omega}_{!}, \underline{\omega}_{*}$ are localisations while $\underline{\omega}^{!}$and $\underline{\omega}^{*}$ are fully faithful. Moreover, if $X \in$ Cor is proper, we have a canonical isomorphism $\underline{\omega}^{*} \mathbb{Z}_{\mathrm{tr}}(X) \simeq \mathbb{Z}_{\mathrm{tr}}(X, \emptyset)$.

Proof. The only non obvious statement is the last claim, which follows from Lemma 1.5.1.

\subsection{MPST and MPST}

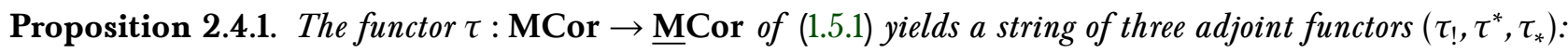

$$
\operatorname{MPST} \underset{\stackrel{\tau_{*}}{\longrightarrow}}{\stackrel{\tau_{!}^{*}}{\longrightarrow}} \text { MPST, }
$$

where $\tau_{!}, \tau_{*}$ are fully faithful and $\tau^{*}$ is a localisation; $\tau_{!}$has a pro-left adjoint $\tau^{!}$, hence is exact. There are natural isomorphisms

$$
\omega_{!} \simeq \underline{\omega}_{!} \tau_{!}, \quad \omega_{*} \simeq \underline{\omega}_{*} \tau_{*}, \quad \omega^{!} \simeq \tau^{!} \underline{\omega}^{!} .
$$

The same holds for the functor $\tau_{s}$ from Theorem 1.5.2. Namely, we have a string of three adjoint functors $\left(\tau_{s !}, \tau_{s}^{*}, \tau_{s *}\right)$ and they satisfy

$$
\omega_{s !} \simeq \underline{\omega}_{s !} \tau_{s !}, \quad \omega_{s *} \simeq \underline{\omega}_{s *} \tau_{s *}, \quad \omega_{s}^{!} \simeq \tau_{s}^{!} \underline{\omega}_{s}^{!} .
$$

Proof. This follows from Theorem 1.5.2 and Proposition A.4.1.

\section{Lemma 2.4.2.}

(1) For $G \in$ MPST, $G^{\prime} \in$ MPS and $M \in \underline{\text { MSm, we have }}$

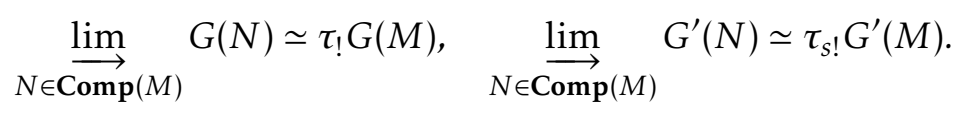

(2) The unit maps $\mathrm{Id} \rightarrow \tau^{*} \tau_{!}$and $\mathrm{Id} \rightarrow \tau_{s}^{*} \tau_{s !}$ are isomorphisms.

(3) There is an natural isomorphism $\tau_{!} \omega^{*} \simeq \underline{\omega}^{*}$. 
Proof. (1) This follows from Lemma 1.8.3, Theorem 1.5.2 and Proposition A.4.1.

(2) This follows from (1) since $\operatorname{Comp}(M)=\{M\}$ for $M \in \mathbf{M S m}$.

(3) For $F \in \mathbf{P S T}$ and $M \in$ MCor, we compute

$$
\tau_{!} \omega^{*} F(M)=\underset{N \in \operatorname{Comp}(M)}{\lim _{\longrightarrow}} \omega^{*} F(N)=\underset{N \in \operatorname{Comp}(M)}{\lim _{\longrightarrow}} F\left(N^{\mathrm{o}}\right)=F\left(M^{\mathrm{o}}\right)=\underline{\omega}^{*} F(M) .
$$

We are done.

Remark 2.4.3. By Lemma 1.8.3 we have the formulas

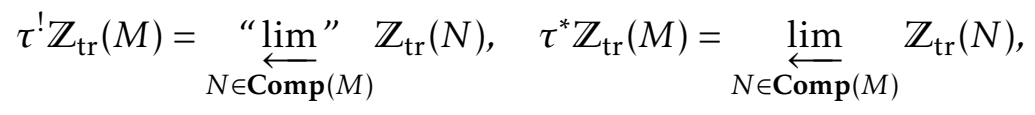

where the latter inverse limit is computed in MPST.

Question 2.4.4. Is $\tau^{!}$exact?

\section{5. $\underline{M P S T}^{\text {fin }}$ and MPST}

Proposition 2.5.1. Let $\underline{b}_{s}: \underline{\operatorname{MSm}}{ }^{\text {fin }} \rightarrow \underline{\text { MSm }}$ and $\underline{b}: \underline{\text { MCor }}^{\text {fin }} \rightarrow \underline{\text { MCor } b e ~ t h e ~ i n c l u s i o n ~ f u n c t o r s ~ f r o m ~(1.9 .1) . ~}$ Then $\underline{b}_{s}$ and $\underline{b}$ yield strings of three adjoint functors $\left(\underline{b}_{s}, \underline{b}_{s}^{*}, \underline{b}_{s *}\right)$ and $\left(\underline{b}_{!}, \underline{b}^{*}, \underline{b}_{*}\right)$ :

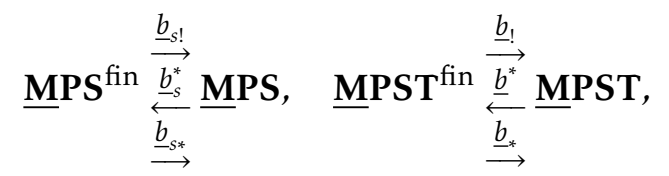

where $\underline{b}_{s !}, \underline{b}_{s *}, \underline{b}_{!}, \underline{b}_{*}$ are localisations; $\underline{b}_{s}^{*}, \underline{b}^{*}$ are exact and fully faithful; $\underline{b}_{s !}, \underline{b}_{!}$have pro-left adjoints, hence are exact. The counit maps $\underline{b}_{s} \underline{\underline{b}_{s}^{*}} \rightarrow \operatorname{Id}$ and $\underline{b}_{\underline{b}} \underline{b}^{*} \rightarrow \operatorname{Id}$ are isomorphisms. For $F_{s} \in \underline{M P S}^{\text {fin }}, F \in \underline{\text { MPST }}{ }^{\text {fin }}$ and $\mathrm{M} \in \mathrm{Ob}(\underline{\mathbf{M S m}})=\mathrm{Ob}(\underline{\mathbf{M C o r}})$, we have (see Def. 1.9.1)

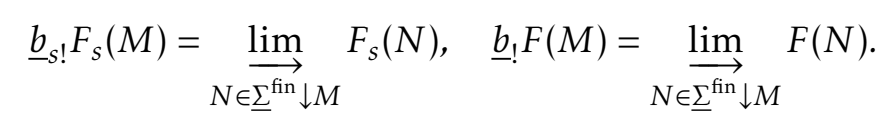

Proof. This follows from the usual yoga applied with Proposition 1.9.2 and Lemma A.3.1.

\subsection{With and without transfers}

Proposition 2.6.1. Let $\underline{\underline{c}}: \underline{\mathrm{MS}} \mathrm{M} \rightarrow \underline{\mathrm{M}} \mathrm{Cor}$ be the functor from (1.3.1). Then $\underline{c}$ yields a string of three adjoint functors $\left(\underline{c}_{!}, \underline{c}^{*}, \underline{c}_{*}\right)$ :

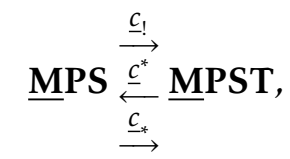

where $\underline{c}^{*}$ is exact and faithful (but not full). We have

$$
\underline{c}_{!} \mathbb{Z}^{p}(M)=\mathbb{Z}_{\mathrm{tr}}(M)
$$

for any $M \in \underline{\mathbf{M S m}}$, where $\mathbb{Z}^{p}(M)$ is ${ }^{4}$ the presheaf $N \mapsto \mathbb{Z}[\underline{\operatorname{MSm}}(N, M)]$.

The same statements hold for $c: \mathrm{MSm} \rightarrow \mathrm{MCor}$ and $\underline{c}^{\mathrm{fin}} ; \underline{\mathrm{MSm}}{ }^{\text {fin }} \rightarrow \underline{\text { MCor }}{ }^{\text {fin }}$ from (1.3.1). Precisely, they yield strings of three adjoint functors $\left(c_{!}, c^{*}, c_{*}\right)$ and $\left(\underline{f}_{!}^{\mathrm{fin}}, \underline{c}^{\mathrm{fin}}, \underline{,}_{*}^{\mathrm{fin}}\right) ; c^{*}$ and $\underline{c}^{\text {fin* }}$ are exact and faithful. (The analogue of (2.6.1) also holds for $c$ and $\underline{\mathrm{c}}^{\mathrm{fin}}$, but we will not need it.)

Proof. To define $\underline{c}_{1}, \underline{c}^{*}$ and $\underline{c}_{*}$, we use the free additive category $\mathbb{Z} \underline{\mathrm{MSm}}$ on $\underline{\mathrm{MSm}}$ [Mac98, Chapter VIII, Section 3, Exercises 5 \& 6]: it comes with a canonical functor $\gamma: \underline{\mathbf{M S m}} \rightarrow \underline{\mathbb{Z}} \underline{\mathbf{M S m}}$ and is 2-universal for contravariant functors to additive categories. In particular:

- The functor $\underline{c}$ induces an additive functor $\underline{\tilde{c}}: \mathbb{Z} \underline{\mathbf{M S m}} \rightarrow \underline{\text { MCor. }}$

\footnotetext{
${ }^{4}$ We put a superscript $p$ to distinguish it from its associated sheaf $\mathbb{Z}(M)$, to be introduced in (4.4.1).
} 
- By the 2-universality, the functor $\gamma$ induces an equivalence $\gamma^{*}: \operatorname{Mod}-\mathbb{Z} \underline{\mathbf{M S m}} \cong \underline{\mathbf{M P S}}$, where

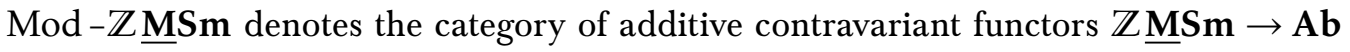

- For $M, N \in \underline{\mathbf{M S m}}$, we have a canonical isomorphism

$$
\mathbb{Z} \underline{\mathbf{M S m}}(\gamma(N), \gamma(M)) \simeq \mathbb{Z}[\underline{\operatorname{MS}} \mathbf{m}(N, M)] .
$$

As usual, $\underline{\tilde{c}}$ induces a string of three adjoint functors $\left(\underline{\tilde{c}}_{!}, \underline{\tilde{c}}^{*}, \underline{\tilde{c}}_{*}\right)$ (see $\left.\S \mathrm{A} .4\right)$. We then define $\underline{c}_{!}$as $\underline{\tilde{c}}_{!} \circ\left(\gamma^{*}\right)^{-1}$, etc. Everything follows from this except the faithfulness of $\underline{c}^{*}$, which is a consequence of the essential surjectivity of $\underline{c}$. The cases of $\underline{c}^{\text {fin }}$ and $c$ are dealt with similarly.

Lemma 2.6.2. (1) We have

$$
\underline{c}^{\text {fin* }} \underline{b}^{*}=\underline{b}_{s}^{*} \underline{c}^{*}, \quad \underline{b}_{!} \underline{c}_{!}^{\text {fin }}=\underline{c} ! \underline{b}_{s !}, \quad \underline{c}^{*} \underline{b}_{!}=\underline{b}_{s !} \underline{c}^{\text {fin* }} .
$$

(2) We have

$$
c^{*} \tau^{*}=\tau_{s}^{*} \underline{c}^{*}, \quad \underline{c}^{*} \tau_{!}=\tau_{s !} c^{*}, \quad \underline{c}^{\mathrm{fin} *} \tau_{!}^{\mathrm{fin}}=\tau_{s !}^{\mathrm{fin}} c^{\mathrm{fin} *} .
$$

Proof. The first two equalities of (1) follows from the equality $\underline{b} \underline{c}^{\text {fin }}=\underline{c} \underline{b}_{s}$ (see (1.9.2)). Similarly, the first equality of (2) follows from $\tau c=\underline{c} \tau_{s}$. By (2.5.1), we have

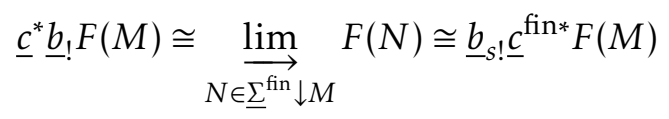

for any $F \in$ MPST $^{\text {fin }}$ and $M \in \underline{\mathbf{M S}}$ S. (Note that all morphisms of $\underline{\Sigma}^{\text {fin }} \downarrow M$ are in $\underline{\mathbf{M S m}}{ }^{\text {fin }}$, and that both of $\underline{b}_{\text {! }}$ and $\underline{b}_{s !}$ can be computed by using the same $\underline{\Sigma}^{\text {fin }} \downarrow M$.) This proves the last formula of (1). Lemma 2.4.2 (1) shows that

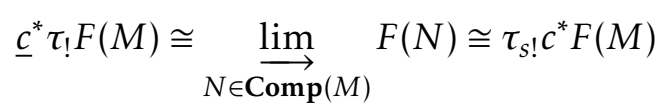

for any $F \in$ MPST and $M \in \underline{\mathbf{M S}}$. The last one of (2) is similar.

\subsection{A patching lemma}

By the previous lemma, we obtain a commutative diagram of categories (cf. (1.9.2)):

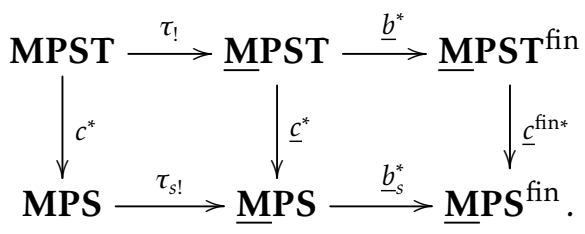

All vertical arrows are faithful and horizontal ones fully faithful.

Lemma 2.7.1. Both squares of (2.7.1) are "2-Cartesian". More precisely, the following assertions hold.

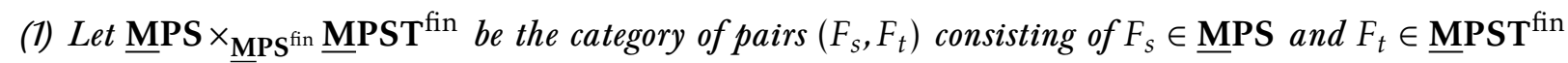
such that their restriction to the common subcategory $\underline{M S}^{\text {fin }}{ }^{\text {are equal. The functor }}$

$$
\underline{\text { MPST }} \rightarrow \underline{\text { MPS }} \times \underline{\text { MPS }}^{\text {fin }} \underline{\text { MPST }}{ }^{\text {fin }},
$$

defined by $F \mapsto\left(\underline{c}^{*} F, \underline{b}^{*} F\right)$ is an equivalence of categories.

(2) Let MPS $\times_{\text {MPS }} \underline{\text { MPST }}$ be the category of triples $\left(F_{s}, F_{t}, \varphi\right)$ consisting of $F_{s} \in$ MPS, $F_{t} \in \underline{\text { MPST }}$ and an isomorphism $\varphi: \tau_{s !} F_{s} \cong \underline{c}^{*} F_{t}$ in $\underline{\text { MPS. The functor }}$

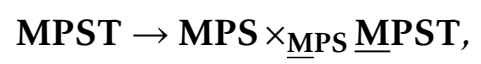

defined by $F \mapsto\left(c^{*} F, \tau_{!} F, \theta_{F}\right)$, where $\theta_{F}: \tau_{s !} c^{*} F \cong \underline{c}^{*} \tau_{!} F$ is from (2.6.3), is an equivalence of categories. 
Proof. (1) is the content of Corollary 1.9.4. We show (2). Given $\left(F_{s}, F_{t}, \varphi\right)$, we shall construct $F \in$ MPST as follows. Set $F(M):=F_{s}(c M)$ for any $M \in$ MCor. Since $M$ is proper, we have an isomorphism

$$
F(M)=F_{s}(c M)=\tau_{s !} F_{s}\left(\tau_{s} c M\right) \stackrel{\varphi_{M}}{\longrightarrow} \underline{c}^{*} F_{t}(\underline{c} \tau M)=F_{t}(\tau M),
$$

which we denote by $\widetilde{\varphi}_{M}$. For $\gamma \in \operatorname{MCor}(M, N)$, we define $F(\gamma):=\widetilde{\varphi}_{M}^{-1} F_{t}(\gamma) \widetilde{\varphi}_{N}$. It is straightforward to see that $\left(F_{s}, F_{t}, \varphi\right) \mapsto F$ gives a quasi-inverse.

\subsection{The functors $n_{!}$and $n^{*}$}

As in $\S$ A.4, the functor $(-)^{(n)}$ of Definition 1.4.1 induces a string of adjoint endofunctors $\left(n_{!}, n^{*}, n_{*}\right)$ of MPST, where $n^{*}$ is given by $n^{*}(F)(M)=F\left(M^{(n)}\right)$. We shall not use $n_{*}$ in the sequel.

Lemma 2.8.1. The functor $n_{!}$is fully faithful.

Proof. This follows formally from the same properties of $(-)^{(n)}$.

Proposition 2.8.2. For any $F \in$ MPST, there is a natural isomorphism

$$
\omega^{*} \omega_{!} F \simeq \infty^{*} F
$$

where $\infty^{*} F(M):=\lim _{\longrightarrow} F\left(M^{(n)}\right)$ (for the natural transformations (1.4.1)).

Proof. Let $M \in$ MCor and $X=\omega M$. Then

$$
\omega^{*} \omega_{!} F(M)=\underset{M^{\prime} \in \lim _{\mathbf{M S m}}}{\operatorname{li}(X)} F\left(M^{\prime}\right),
$$

and the claim follows from Lemma 1.7.4.

Proposition 2.8.3. For all $n \geq 1$, the natural transformation $\omega_{!} \rightarrow \omega_{!} n^{*}$ stemming from (1.4.1) is an isomorphism.

Proof. Let $F \in$ MPST. For $X \in$ Cor, we have

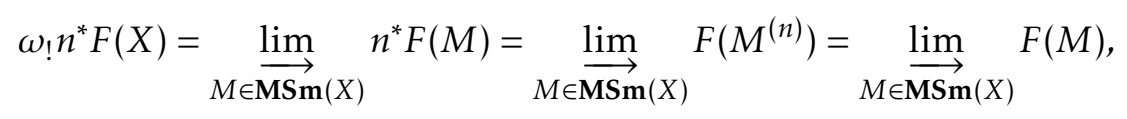

where the last isomorphism follows from Lemma 1.7.4.

\section{Sheaves on $\underline{M S m}^{\text {fin }}$ and $\underline{\mathrm{MCor}}{ }^{\text {fin }}$}

\subsection{Nisnevich topology on $\underline{\mathrm{MSm}}{ }^{\text {fin }}$}

Definition 3.1.1. We call a morphism $p: U \rightarrow M$ in $\underline{\mathbf{M S m}}^{\text {fin }}$ a Nisnevich cover if

(i) $\bar{p}: \bar{U} \rightarrow \bar{M}$ is a Nisnevich cover of $\bar{M}$ in the usual sense;

(ii) $p$ is minimal (that is, $U^{\infty}=\bar{p}^{*}\left(M^{\infty}\right)$ ).

Since the morphisms appearing in the Nisnevich covers are squarable by Corollary 1.10 .7 (1), we obtain a Grothendieck topology on $\underline{\mathbf{M S}}{ }^{\text {fin }}$. The category $\underline{\mathbf{M S m}}^{\text {fin }}$ endowed with this topology will be called the big Nisnevich site of $\underline{\mathbf{M S m}}{ }^{\text {fin }}$ and denoted by $\underline{\mathbf{M S}} \mathbf{m}_{\mathrm{Nis}}^{\text {fin }}$.

Definition 3.1.2. Let us fix $M \in \underline{\mathbf{M S m}}{ }^{\text {fin }}$. Let $M_{\mathrm{Nis}}$ be the category of minimal morphisms $f: N \rightarrow M$ in $\underline{\mathbf{M S m}}^{\text {fin }}$ such that $\bar{f}$ is étale, endowed with the topology induced by $\underline{\mathbf{M S}} \mathbf{m}_{\mathrm{Nis}}^{\mathrm{fin}}$.

The following lemma is obvious from the definitions: 
Lemma 3.1.3. Let $M \in \underline{M S m}^{\text {fin }}$. Let $(\bar{M})_{\text {Nis }}$ be the (usual) small Nisnevich site on $\bar{M}$. Then we have an isomorphism of sites

$$
M_{\mathrm{Nis}} \rightarrow(\bar{M})_{\mathrm{Nis}}, \quad N \mapsto \bar{N},
$$

whose inverse is given by $(p: X \rightarrow \bar{M}) \mapsto\left(X, p^{*}\left(M^{\infty}\right)\right)$. (This isomorphism of sites depends on the choice of $M^{\infty}$.)

Lemma 3.1.4. Let $\alpha: M \rightarrow N$ be a morphism in $\underline{\mathbf{M C o r}^{\text {fin }}}$ and let $p: U \rightarrow N$ be a Nisnevich over in $\underline{\mathbf{M S m}}{ }^{\text {fin }}$. Then there is a commutative diagram

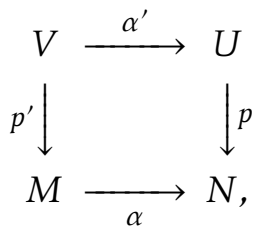

where $\alpha^{\prime}: V \rightarrow U$ is a morphism in $\underline{\text { MCor }}{ }^{\text {fin }}$ and $p^{\prime}: V \rightarrow M$ is a Nisnevich cover in $\underline{\mathbf{M S m}}{ }^{\text {fin }}$.

Proof. We may assume $\alpha$ is integral. Let $\bar{\alpha}$ be the closure of $\alpha$ in $\bar{M} \times \bar{N}$. Since $\bar{\alpha}$ is finite over $\bar{M}$, we may find a Nisnevich cover $p^{\prime}: \bar{V} \rightarrow \bar{M}$ such that $\tilde{p}$ in the diagram (all squares being cartesian)

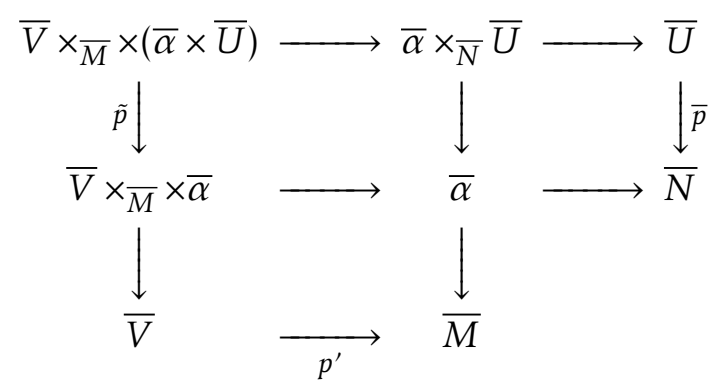

has a splitting $s$. Put $V:=\left(\bar{V}, p^{\prime *}\left(M^{\infty}\right)\right) \in \underline{\mathbf{M S}} \mathbf{m}$. The image of $s$ gives us a desired correspondence $\alpha^{\prime}$.

Remark 3.1.5. One can also define the Zariski and étale topologies on $\underline{\mathbf{M S}} \mathbf{m}^{\text {fin }}$. Most results of this section (notably Theorems 3.4.1, 3.5.3, and Corollary 3.5.6) remain true for the étale topology, but not for the Zariski topology (e.g. Lemma 3.1.4 already fails for it).

However, from the next section onward we will make essential use of cd-structures. As the étale topology cannot be defined by a cd-structure, we decide to stick to the Nisnevich topology from the beginning.

\subsection{A cd-structure on $\underline{\mathrm{MS}} \mathrm{m}^{\text {fin }}$}

Let Sq be the product category of $[0]=\{0 \rightarrow 1\}$ with itself, depicted as

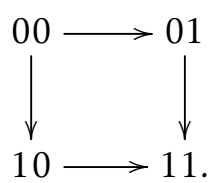

For any category $\mathcal{C}$, denote by $\mathcal{C}^{\mathbf{S q}}$ for the category of functors from Sq to $\mathcal{C}$. A functor $f: \mathcal{C} \rightarrow \mathcal{C}^{\prime}$ induces a functor $f^{\mathrm{Sq}}: \mathcal{C}^{\mathbf{S q}} \rightarrow \mathcal{C}^{\prime \mathbf{S q}}$.

We refer to §A.7 for the notion of cd-structure, and its properties.

\section{Definition 3.2.1.}

(1) A Cartesian square

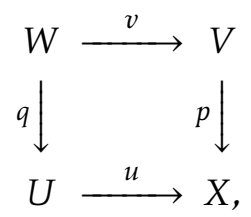


in Sch is called an elementary Nisnevich square if $p$ is étale, $p^{-1}(X \backslash U)_{\mathrm{red}} \rightarrow(X \backslash U)_{\mathrm{red}}$ is an isomorphism and $u$ is an open embedding,. In this situation, we say $U \sqcup V \rightarrow X$ is an elementary Nisnevich cover. Recall that an additive presheaf is a Nisnevich sheaf if and only if it transforms any elementary Nisnevich square into a cartesian square [Voe10a, Corollary 2.17], [Voe10b, Theorem 2.2].

(2) A diagram (3.2.1) in $\underline{\mathbf{M S m}}^{\text {fin }}$ is called an $\underline{\mathrm{MV}}^{\text {fin }}$-square if all morphisms are minimal and it becomes an elementary Nisnevich square (in Sch) after applying the forgetful functor of Definition 1.3.3 (2).

Lemma 3.2.2. $A \underline{\mathrm{MV}}^{\mathrm{fin}}$-square (3.2.1) is cartesian in $\underline{\mathrm{MSm}}^{\mathrm{fin}}$.

Proof. The last part of Proposition 1.10.4 (2) shows that no irreducible component of $\bar{X}$ has its image inside $\left|U^{\infty}\right|$ or $\left|V^{\infty}\right|\left(\right.$ i.e. $\bar{W}_{1}$ in loc. cit. agrees with $\left.\bar{W}\right)$, and then Proposition 1.10.4 (1) shows that $X$ is the fiber product since $q^{*} U^{\infty}=v^{*} V^{\infty}=W^{\infty}$ by minimality.

Proposition 3.2.3. The following assertions hold.

(1) The topology on $\underline{\mathrm{MS}} \mathrm{m}_{\mathrm{Nis}}^{\text {fin }}$ (cf. Def. 3.1.1) coincides with the topology associated with the cd-structure $P_{\underline{\mathrm{MV}}}{ }^{\text {fin }}$ consisting of $\underline{\mathrm{MV}}^{\mathrm{fin}}$-squares.

(2) The cd-structure $P_{\mathrm{MV}^{\text {fin }}}$ is strongly complete and strongly regular in the sense of Definition A.7.4, hence complete and regular in the sense of [Voe10a] (cf. Definition A.7.1).

Proof. (1) follows from Lemma 3.1.3 and [Voe10b, Remark after Proposition 2.17]. The first assertion of (2) follows from (the proof of) [Voe10b, Theorem 2.2]. The second assertion of (2) follows from [Voel0a, Lemma 2.5, Lemma 2.11]

\subsection{Sheaves on $\underline{\mathrm{MSm}} \mathrm{m}^{\text {fin }}$}

Definition 3.3.1. We define $\underline{M N S}^{\text {fin }}$ to be the full subcategory of $\underline{M P S}^{\text {fin }}$ consisting of Nisnevich sheaves.

Theorem 3.3.2. Let $F \in \underline{\mathbf{M N S}}{ }^{\text {fin }}$. Then $H_{\mathrm{Nis}}^{i}(X, F)=0$ for any $X \in \underline{\mathbf{M S m}}^{\text {fin }}$ and $i>\operatorname{dim} X$ (where $\operatorname{dim} X$ is defined as $\left.\operatorname{dim} X:=\operatorname{dim} X^{\mathrm{o}}=\operatorname{dim} \bar{X}\right)$.

Proof. This is clear from Lemma 3.1.3 and the known properties of the Nisnevich site.

Definition 3.3.3. An additive functor $F$ between additive categories is strongly additive if it commutes with infinite direct sums.

This property is not used in the present paper, but it will be essential in [KMSY21] when we deal with unbounded derived categories.

Lemma 3.3.4. The category $\underline{M N S}^{\text {fin }}$ is closed under infinite direct sums and the inclusion functor denoted by $\underline{\mathrm{f}}_{-s \mathrm{fin}}^{\mathrm{fin}}: \underline{\mathrm{MNS}}^{\mathrm{fin}} \rightarrow \underline{\text { MPS }}^{\mathrm{fin}}$ is strongly additive.

Proof. Indeed, the sheaf condition is tested on finite diagrams, hence the presheaf given by a direct sum of sheaves is a sheaf (small filtered colimits commute with finite limits, [Mac98, Chapter IX, Section 2, Theorem 1]).

Proposition 3.3.5. For any $M \in \underline{\mathrm{MS}} \mathrm{Sm}$ we have

$$
\underline{c}^{\mathrm{fin} *} \mathbb{Z}_{\mathrm{tr}}^{\mathrm{fin}}(M), \quad \underline{c}^{\mathrm{fin} *} \underline{b}^{*} \mathbb{Z}_{\mathrm{tr}}(M) \in \underline{\mathrm{MN}} \mathbf{S}^{\mathrm{fin}},
$$

where $\mathbb{Z}_{\mathrm{tr}}^{\mathrm{fin}}, \mathbb{Z}_{\mathrm{tr}}$ are the representable presheaves (notation 2.1.2) and the functors $\underline{b}^{*}$ and $\underline{c}^{\text {fin* }}$ are from Propositions 2.5.1, 2.6.1. 
Proof. We show the stronger statement that $\mathbb{Z}_{\mathrm{tr}}(M)$ restricts to an étale sheaf on $\underline{N}_{\text {ét }}$ for any $N \in \underline{\text { MCor }}{ }^{\text {fin }}$. Let $p: \bar{U} \rightarrow \bar{N}$ be an étale cover and let $U:=\left(\bar{U}, p^{*} N^{\infty}\right)$. We have a commutative diagram

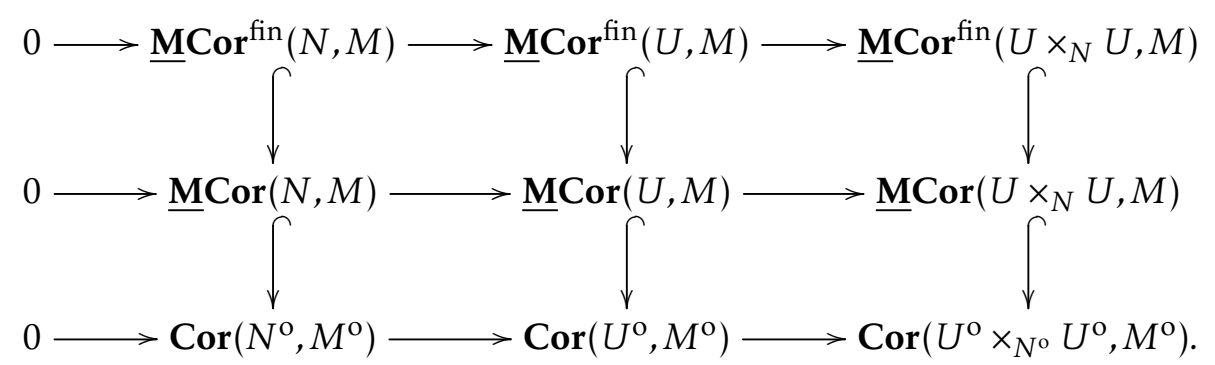

The bottom row is exact by [MVW06, Lemma 6.2]. The exactness of the top and middle row now follows from Lemma 1.9.6.

\section{4. Čech complex}

Let $p: U \rightarrow M$ be a Nisnevich cover in $\underline{\mathbf{M S m}}{ }^{\text {fin }}$. We write $U \times_{M} U$ for the modulus pair corresponding to $\bar{U} \times \bar{M} \bar{U}$ under the isomorphism of sites from Lemma 3.1.3. Note that it is a fibre product in $\underline{\mathbf{M S m}}^{\text {fin }}$ and in

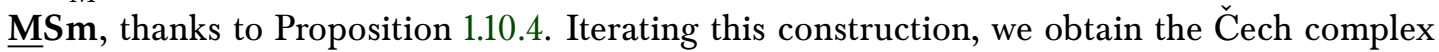

$$
\cdots \rightarrow \underline{c}^{\text {fin* }} \mathbb{Z}_{\text {tr }}^{\text {fin }}\left(U \times \times_{M} U\right) \rightarrow \underline{c}^{\text {fin* }} \mathbb{Z}_{\text {tr }}^{\text {fin }}(U) \rightarrow \underline{c}^{\text {fin* }} \mathbb{Z}_{\text {tr }}^{\text {fin }}(M) \rightarrow 0
$$

in $\underline{\mathrm{MNS}} \mathrm{S}^{\mathrm{fin}}$.

Theorem 3.4.1. The complex (3.4.1) is exact in $\underline{\mathrm{MNS}^{\mathrm{fin}}}$.

Remark 3.4.2. This result will be refined several times, see Corollary 3.5.6 and Theorem 4.5.7. Its proof is adapted from [Voe00, Proposition 3.1.3].

Before starting the proof of Theorem 3.4.1, it is convenient to generalize the notion of relative cycles to the modulus setting.

Definition 3.4.3. Let $S=(\bar{S}, D), Z=\left(\bar{Z}, Z^{\infty}\right)$ be two pairs formed of a scheme and an effective Cartier divisor, and let $f: \bar{Z} \rightarrow \bar{S}$ be a morphism. (We don't put any regularity requirement on $\bar{S}-|D|$ or $\bar{Z}-\left|Z^{\infty}\right|$.) We write $L(Z / S)$ for the free abelian group with basis the closed integral subschemes $T \subset \bar{Z}$ such that $T$ is finite and surjective over an irreducible component of $\bar{S}$ and $\left.D\right|_{T^{N}} \geq\left. Z^{\infty}\right|_{T^{N}}$, where $T^{N} \rightarrow T$ is normalization and $\left.(-)\right|_{T^{N}}$ denotes pull-back of Cartier divisors.

Example 3.4.4. If $S$ is a modulus pair and $M=\left(\bar{M}, M^{\infty}\right)$ is another modulus pair, then we have a canonical isomorphism $\operatorname{MCor}^{\mathrm{fin}}(S, M) \simeq L(\bar{S} \times M / S)$, where $\bar{S} \times M$ is the modulus pair $\left(\bar{S} \times \bar{M}, \bar{S} \times M^{\infty}\right)$ : this follows from Remark 1.3.5 (3).

Define a category $\mathcal{D}(S)$ as follows: objects are pairs $(Z, f)$ as in Definition 3.4.3. A morphism in $\mathcal{D}(S)$, $(Z, f) \rightarrow\left(Z^{\prime}, f^{\prime}\right)$, is a minimal morphism $\varphi: Z \rightarrow Z^{\prime}$ such that $f=f^{\prime} \circ \bar{\varphi}$. Composition is obvious.

Lemma 3.4.5. The push-forward of cycles makes $(Z, f) \mapsto L(Z / S)$ a covariant functor on $\mathcal{D}(S)$.

Proof. Let $\varphi:(Z, f) \rightarrow\left(Z^{\prime}, f^{\prime}\right)$ be a morphism in $\mathcal{D}(S)$, and let $T \in L(Z / S)$. Then $\varphi(T)$ is still finite and surjective over a component of $\bar{S}$ [MVW06, Lemma 1.4]. Moreover, it still verifies the modulus condition: this follows from the minimality of $\varphi$ and from Lemma 1.2.1. We set as usual $\varphi_{*} T=[k(T): k(\varphi(T))] \varphi(T)$ : this defines $\varphi_{*}: L(Z / S) \rightarrow L\left(Z^{\prime} / S\right)$, and the functoriality $(\psi \circ \varphi)_{*}=\psi_{*} \circ \varphi_{*}$ is obvious.

Proof of Theorem 3.4.1. In view of Lemma 3.1.3, it suffices to show the exactness of (3.4.1) evaluated at $S$

$$
\cdots \rightarrow \underline{\operatorname{MCor}}{ }^{\mathrm{fin}}\left(S, U \times \times_{M} U\right) \rightarrow \underline{\operatorname{MCor}}{ }^{\mathrm{fin}}(S, U) \rightarrow \underline{\operatorname{MCor}}^{\mathrm{fin}}(S, M) \rightarrow 0
$$


for the henselisation $S=(\bar{S}, D)$ of any modulus pair $N=\left(\bar{N}, N^{\infty}\right)$ at any point of $\bar{N}$. As in [Voe00], the strategy is to write (3.4.2) as a filtered colimit of contractible chain complexes.

Write $\mathcal{E}(S, M)$ for the collection of integral closed subsets of $S^{\circ} \times M^{\mathrm{o}}$ which belong to $\operatorname{MCor}^{\mathrm{fin}}(S, M)$ (this is the canonical basis of $\underline{\operatorname{MCor}}{ }^{\text {fin }}(S, M)$ ). Let $\mathcal{C}(M)$ be the set of closed subschemes of $\overline{\bar{S}} \times \bar{M}$ that are quasi-finite over $\bar{S}$ and not contained in $\bar{S} \times M^{\infty}$, viewed as an (ordered, cofiltered) category. To $Z \in \mathcal{C}(M)$ we associate the subset $\mathcal{E}(Z) \subset \mathcal{E}(S, M)$ of those $F$ such that $F \subset Z$.

Provide $Z \in \mathcal{C}(M)$ with the minimal modulus structure induced by the projection $Z \rightarrow \bar{M}$ (in a sense slightly generalized from Remark 1.1.2 (4), as in Definition 3.4.3: the open subset $Z-Z^{\infty}$ is not necessarily smooth). This yields a functor

$$
\mathcal{C}(M) \rightarrow \mathcal{D}(S)
$$

where $\mathcal{D}(S)$ is the category defined above. In particular, we have a subgroup $L(Z / S) \subset \underline{\operatorname{MCor}}{ }^{\text {fin }}(S, M)$ : it is the free abelian group on $\mathcal{E}(Z)$.

Let $u: M^{\prime} \rightarrow M$ be an étale morphism in $\underline{\mathbf{M S m}}^{\text {fin }}$, as in Definition 3.1.2. For $Z \in \mathcal{C}(M)$, define $u^{*} Z=Z \times \bar{M} \bar{M}^{\prime}$. Then $u^{*} Z \in \mathcal{C}\left(M^{\prime}\right)$, and there is a commutative diagram

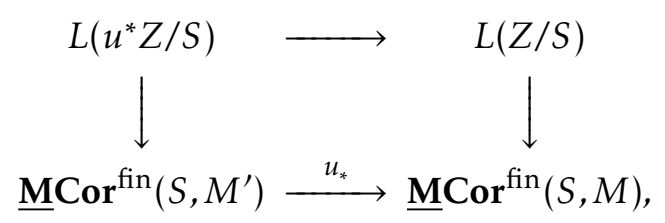

where the bottom horizontal map is composition by the graph of $u$. This yields subcomplexes

$$
\cdots \rightarrow L(Z \times \bar{M}(\bar{U} \times \bar{M} \bar{U})) \rightarrow L(Z \times \bar{M} \bar{U}) \rightarrow L(Z) \rightarrow 0
$$

of (3.4.2), for $Z \in \mathcal{C}(M)$.

Let $\mathcal{C}_{f}(M) \subset \mathcal{C}(M)$ be the subset of those $Z$ which are finite over $\bar{S}$. It is a filtered subcategory, and we have

$$
\mathcal{E}\left(S, M^{\prime}\right)=\bigcup_{Z \in \mathcal{C}_{f}(M)} \mathcal{E}\left(u^{*} Z\right) .
$$

Indeed, for $Z^{\prime} \in \mathcal{C}\left(M^{\prime}\right)$, let $Z=\overline{\left(\operatorname{Id}_{\bar{S}} \times \bar{u}\right)\left(Z^{\prime}\right)}$ and let $Z_{f}=\bigcup_{F \in \mathcal{E}(Z)} F$. Then $\mathcal{E}\left(Z^{\prime}\right) \subset \mathcal{E}\left(u^{*} Z_{f}\right)$ since $\left(\operatorname{Id}_{\bar{S}} \times \bar{u}\right)(F)$ is finite over $\bar{S}$ for $F \in \mathcal{E}\left(Z^{\prime}\right)$.

This proves that (3.4.2) is obtained as the filtered inductive limit of the complexes (3.4.3) when $Z$ ranges over $\mathcal{C}_{f}(M)$. It suffices to show the exactness of (3.4.3) for such a $Z$.

Since $Z$ is finite over the henselian local scheme $\bar{S}, Z$ is a disjoint union of henselian local schemes. Thus the Nisnevich cover $Z \times \bar{M} \bar{U} \rightarrow Z$ admits a section $s_{0}: Z \rightarrow Z \times \bar{M} \bar{U}$. Define for $k \geq 1$

$$
s_{k}:=s_{0} \times \bar{M}_{\bar{M}} \operatorname{Id}_{\bar{U}^{k}}: Z \times \bar{M}_{\bar{U}} \bar{U}^{k} \rightarrow Z \times \bar{M} \bar{U} \times \bar{M}_{\bar{U}}^{k}=Z \times \bar{M}_{\bar{M}} \bar{U}^{k+1}
$$

where $\bar{U}^{k}:=\bar{U} \times \bar{M} \cdots \times \bar{M} \bar{U}$. Then the maps

$$
L\left(Z \times \bar{M}^{k}\right) \rightarrow L\left(Z \times \bar{M}^{k+1}\right)
$$

induced by $s_{k}$ via Lemma 3.4.5 give us a homotopy from the identity to zero.

\subsection{Sheafification preserves finite transfers}

Let $\underline{a}_{s \mathrm{Nin}}^{\mathrm{fin}}: \underline{\text { MPS }}^{\mathrm{fin}} \rightarrow \underline{\text { MNS }}^{\text {fin }}$ be the sheafification functor, that is, the left adjoint of the inclusion functor $\underline{i}_{s \mathrm{Nis}}^{\mathrm{fin}}: \underline{\mathrm{MNS}}^{\mathrm{fin}} \hookrightarrow \underline{\text { MPS }}^{\text {fin }}$. It exists for general reasons and is exact [SGA4, exposé II, théorème 3.4].

Definition 3.5.1. Let $\underline{\text { MNST }}^{\text {fin }}$ be the full subcategory of $\underline{\text { MPST }}^{\text {fin }}$ consisting of all objects $F \in \underline{M P S T}^{\text {fin }}$ such that $\underline{c}^{\text {fin* }} F \in \underline{\mathbf{M N S}}{ }^{\text {fin }}$ (see Proposition 2.6.1 for $\underline{c}^{\text {fin*) }}$ ). 
Lemma 3.5.2. The category MNST $^{\mathrm{fin}}$ is closed under infinite direct sums in MPST $^{\mathrm{fin}}$, and the inclusion functor $\underline{i}_{\mathrm{Nis}}^{\mathrm{fin}}: \underline{\text { MNST }}^{\mathrm{fin}} \rightarrow \underline{\text { MPST }}^{\mathrm{fin}}$ is strongly additive (Definition 3.3.3). The objects $\mathbb{Z}_{\mathrm{tr}}^{\mathrm{fin}}(M)$ and $b^{*} \mathbb{Z}_{\mathrm{tr}}(M)$ belong to $\underline{\mathrm{MNST}} \mathrm{T}^{\text {fin }}$ for any $M \in \underline{\mathrm{MC}}$ Cor.

Proof. This follows from Lemma 3.3.4, because $\underline{c}^{\text {fin* }}$ is strongly additive as a left adjoint. The last claim follows from Proposition 3.3.5.

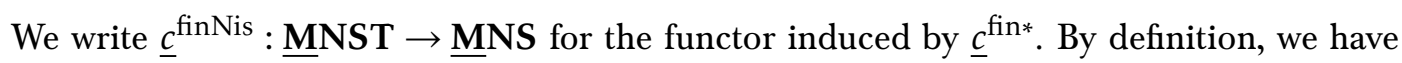

$$
\underline{c}^{\mathrm{fin} *} \underline{i}_{\mathrm{Nis}}^{\mathrm{fin}}=\underline{i}_{s \mathrm{Nis}} \underline{\mathrm{f}}^{\mathrm{finNis}} \text {. }
$$

Theorem 3.5.3. The following assertions hold.

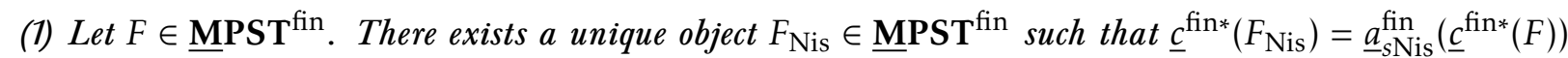
and such that the canonical morphism $u: \underline{c}^{\mathrm{fin} *}(F) \rightarrow \underline{a}_{s \mathrm{Nis}}^{\mathrm{fin}}\left(\underline{c}^{\mathrm{fin} *}(F)\right)=\underline{c}^{\mathrm{fin} *}\left(F_{\mathrm{Nis}}\right)$ extends to a morphism in MPST $^{\text {fin }}$.

(2) The functor $\underline{i}_{\mathrm{Nis}}^{\mathrm{fin}}$ has an exact left adjoint $\underline{a}_{\mathrm{Nis}}^{\mathrm{fin}}: \underline{\mathrm{MPST}}^{\mathrm{fin}} \rightarrow \underline{\mathrm{MNST}}^{\mathrm{fin}}$ satisfying

$$
\underline{c}^{\mathrm{finNis}} \underline{a}_{\mathrm{Nis}}^{\mathrm{fin}}=\underline{a}_{s \mathrm{Nis}}^{\mathrm{fin}} \underline{c}^{\mathrm{fin} *} .
$$

In particular the category $\underline{\mathrm{MNST}}^{\mathrm{fin}}$ is Grothendieck (\$A.10).

(3) The functor $\underline{c}^{\text {finNis }}$ has a left adjoint $\underline{\underline{c}}_{\mathrm{Nis}}^{\mathrm{fin}}=\underline{a}_{\mathrm{Nis}}^{\mathrm{fin}} \underline{c}_{!}^{\mathrm{fin}} \underline{i}_{-\mathrm{Nis}}^{\mathrm{fin}}$. Moreover, $\underline{c}^{\mathrm{finNis}}$ is exact, strongly additive (Definition 3.3.3), and faithful.

Proof. This can be shown by a rather trivial modification of [Voe00, Theorem 3.1.4], but for the sake of completeness we include a proof. To ease the notation, put $F^{\prime}:=\underline{a}_{s \mathrm{Nis}} \underline{c}^{\mathrm{fin}}{ }^{\mathrm{fi}} F \in \underline{\mathrm{MPS}}{ }^{\mathrm{fin}}$. First we construct a homomorphism

$$
\Phi_{M}: F^{\prime}(M) \rightarrow \underline{\operatorname{MPS}}^{\mathrm{fin}}\left(\underline{c}^{\mathrm{fin} *} \mathbb{Z}_{\mathrm{tr}}^{\mathrm{fin}}(M), F^{\prime}\right)
$$

for any $M \in \underline{\mathbf{M S}}$. Take $f \in F^{\prime}(M)$. There exists a Nisnevich cover $p: U \rightarrow M$ in $\underline{\mathbf{M S m}}^{\text {fin }}$ and $g \in \underline{c}^{\text {fin* }} F(U)=F(U)$ such that $\left.f\right|_{U}=u(g)$ in $F^{\prime}(U)$. There also exists a Nisnevich cover $W \rightarrow U \times_{M} U$ such that $\left.g\right|_{W}=0$ in $F(W)$. We have $\underline{a}_{s \mathrm{Nis}}^{\text {fin }} \underline{c}^{\mathrm{fin} *} \mathbb{Z}_{\mathrm{tr}}^{\mathrm{fin}}(M)=\underline{c}^{\mathrm{fin} *} \mathbb{Z}_{\mathrm{tr}}^{\mathrm{fin}}(M)$ because $\underline{c}^{\mathrm{fin} *} \mathbb{Z}_{\mathrm{tr}}^{\mathrm{fin}}(M) \in \underline{\text { MPS }} S_{\mathrm{Nis}}^{\mathrm{fin}}$ by Proposition 3.3.5. Thus we get a commutative diagram in which the horizontal maps are induced by $\underline{a}_{s \mathrm{Nis}}^{\text {fin }} \underline{\underline{c}}{ }_{\text {fin }}$

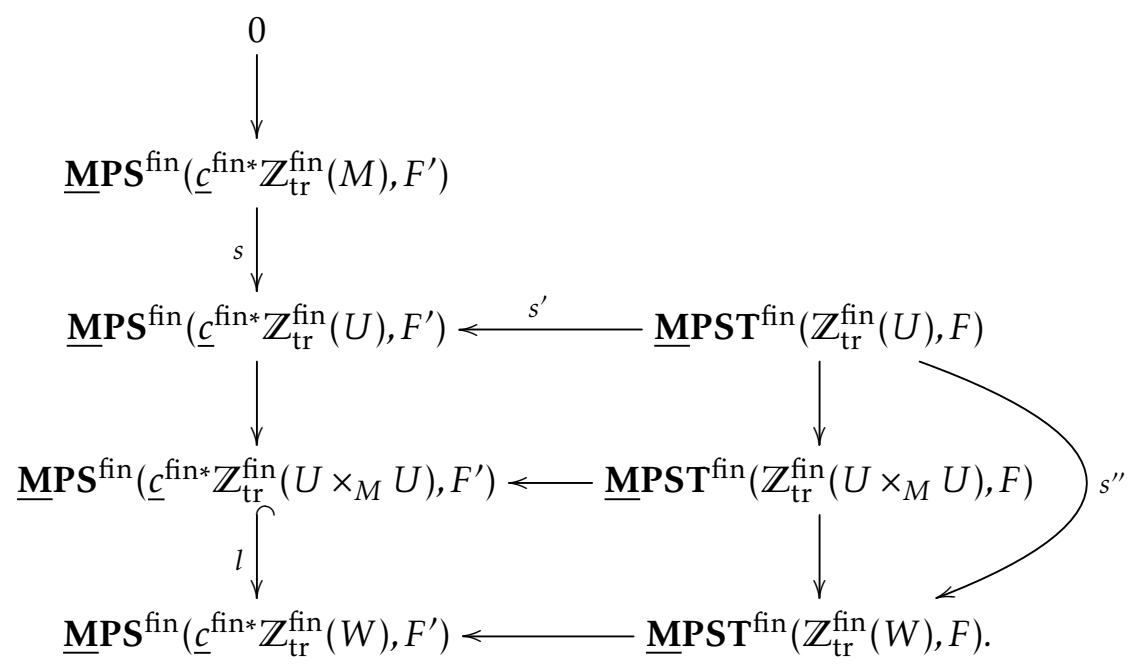

Since $F^{\prime}$ is a sheaf, Theorem 3.4.1 implies that the left vertical column is exact except at the last spot, and that the map $l$ is injective. Since $g \in F(U)=\underline{\operatorname{MPST}^{f i n}}\left(\mathbb{Z}_{\mathrm{tr}}^{\mathrm{fin}}(U), F\right)$ satisfies $s^{\prime \prime}(g)=\left.g\right|_{W}=0$, there exists a unique $h \in \underline{\text { MPS }}{ }^{\text {fin }}\left(c^{*} Z_{\operatorname{tr}}^{\text {fin }}(M), F^{\prime}\right)$ such that $s(h)=s^{\prime}(g)$. One checks that $h$ does not depend on the choices of $p: U \rightarrow M, g \in F(U)$ and $W \rightarrow U \times_{M} U$ by taking a refinement of covers. We define $\Phi_{M}(f):=h$. 
Now we define $G$. On objects we put $G(M)=F^{\prime}(M)$ for $M \in \underline{\mathbf{M S m}}$. For $\alpha \in \underline{\operatorname{MCor}}^{\mathrm{fin}}(M, N)$, we define $\alpha^{*}: F^{\prime}(N) \rightarrow F^{\prime}(M)$ as the composition of

$$
F^{\prime}(N) \stackrel{\Phi_{N}}{\longrightarrow} \underline{\operatorname{MPS}} S^{\mathrm{fin}}\left(\underline{c}^{\mathrm{fin} *} \mathbb{Z}_{\mathrm{tr}}^{\mathrm{fin}}(N), F^{\prime}\right) \longrightarrow \underline{\operatorname{MPS}}^{\mathrm{fin}}\left(\underline{c}^{\mathrm{fin} *} \mathbb{Z}_{\mathrm{tr}}^{\mathrm{fin}}(M), F^{\prime}\right) \longrightarrow F^{\prime}(M),
$$

where the middle map is induced by $\underline{c}^{\mathrm{fin} *}(\alpha): \underline{c}^{\mathrm{fin} *} \mathbb{Z}_{\mathrm{tr}}^{\mathrm{fin}}(M) \rightarrow \underline{c}^{\mathrm{fin} *} \mathbb{Z}_{\mathrm{tr}}^{\mathrm{fin}}(N)$, and the last map is given by $f \mapsto f_{M}\left(\operatorname{Id}_{M}\right)$. One checks that, with this definition, $G$ becomes an object of MPST $^{\text {fin }}$.

To prove uniqueness, take $G, G^{\prime} \in \underline{M P S T}^{\text {fin }}$ which enjoy the stated properties. For any $M \in \underline{\mathbf{M S m}}$ we have $G(M)=G^{\prime}(M)=F^{\prime}(M)$. We also have $G\left(\underline{c}^{\mathrm{fin} *}(q)\right)=G^{\prime}\left(\underline{c}^{\mathrm{fin} *}(q)\right)=F^{\prime}(q)$ for any morphism $q$ in $\underline{\mathbf{M S m}}{ }^{\text {fin }}$. Let $\alpha: M \rightarrow N$ be a morphism in $\underline{\text { MCor }}{ }^{\text {fin }}$ and let $f \in F^{\prime}(N)$. Take a Nisnevich cover $p: U \rightarrow N$ of $\underline{\mathbf{M S m}}^{\text {fin }}$ and $g \in \underline{c}^{\mathrm{fin} *} F(U)=F(U)$ such that $\left.f\right|_{U}=u(g)$ in $F^{\prime}(U)$. Apply Lemma 3.1.4 to get a morphism

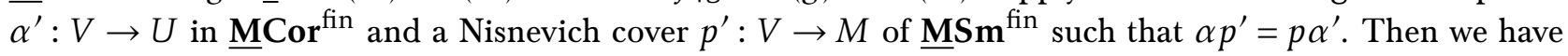

$$
\begin{aligned}
G\left(p^{\prime}\right) G(\alpha)(f) & =G\left(\alpha^{\prime}\right) G(p)(f)=G\left(\alpha^{\prime}\right)(u(g))=u\left(F\left(\alpha^{\prime}\right)(g)\right) \\
& =G^{\prime}\left(\alpha^{\prime}\right)(u(g))=G^{\prime}\left(\alpha^{\prime}\right) G^{\prime}(p)(f)=G^{\prime}\left(p^{\prime}\right) G^{\prime}(\alpha)(f)=G\left(p^{\prime}\right) G^{\prime}(\alpha)(f) .
\end{aligned}
$$

Since $p^{\prime}: V \rightarrow M$ is a Nisnevich cover and $G$ is separated, this implies $G(\alpha)(f)=G^{\prime}(\alpha)(f)$. This completes the proof or (1).

(2) is a consequence of (1) and the fact that MPST ${ }^{\text {fin }}$ is Grothendieck as a category of modules (see Theorem A.10.1 d)). Then (3) follows from Lemma A.8.1.

Remark 3.5.4. A different argument may be given by mimicking the proof of [Ayo15, Corollary 2.2.26].

Definition 3.5.5. An additive functor $\varphi: \mathcal{C} \rightarrow \mathcal{C}^{\prime}$ between abelian categories is faithfully exact if a complex $F^{\prime} \rightarrow F \rightarrow F^{\prime \prime}$ is exact if and only if $\varphi F^{\prime} \rightarrow \varphi F \rightarrow \varphi F^{\prime \prime}$ is.

This happens if $\varphi$ is exact and either faithful or conservative. By Theorems 3.5.3 and 3.4.1, we get:

Corollary 3.5.6. The functor $\underline{\underline{f}}^{\mathrm{finNis}}$ is faithfully exact. In particular, if $p: U \rightarrow M$ is a Nisnevich cover in $\mathrm{MSm}^{\text {fin }}$, then the Cech complex

$$
\cdots \rightarrow \mathbb{Z}_{\mathrm{tr}}^{\mathrm{fin}}\left(U \times_{M} U\right) \rightarrow \mathbb{Z}_{\mathrm{tr}}^{\mathrm{fin}}(U) \rightarrow \mathbb{Z}_{\mathrm{tr}}^{\mathrm{fin}}(M) \rightarrow 0
$$

is exact in MNST $^{\text {fin }}$.

\subsection{Cohomology in $\underline{M N S T}^{\text {fin }}$}

Notation 3.6.1. Let $M \in \underline{\mathbf{M S}} \mathbf{m}^{\text {fin }}$ and let $F \in \underline{\mathbf{M N S}}^{\text {fin }}$ (resp. $F \in \underline{\mathbf{M N S T}^{\text {fin }}}$ ). We write $F_{M}$ for the sheaf on $(\bar{M})_{\text {Nis }}$ induced from $F$ (resp. $\underline{c}^{\text {fino }} F$ ) via the isomorphism of sites from Lemma 3.1.3. (Note that $F_{M}$ depends not only on $\bar{M}$, but also on $M^{\infty}$.) We thus have canonical isomorphisms

$$
\begin{aligned}
& H_{\mathrm{Nis}}^{i}(M, F) \simeq H_{\mathrm{Nis}}^{i}\left(\bar{M}, F_{M}\right), \\
& H_{\mathrm{Nis}}^{i}\left(M, \underline{c}^{\text {finNis }} F\right) \simeq H_{\mathrm{Nis}}^{i}\left(\bar{M}, F_{M}\right),
\end{aligned}
$$

where the right hand sides denote the cohomology of the (usual) small site $(\bar{M})_{\mathrm{Nis}}$.

\section{Definition 3.6.2.}

(1) Let $S$ be a scheme. We say a sheaf $F$ on $S_{\mathrm{Nis}}$ is flasque if $F(V) \rightarrow F(U)$ is surjective for any open dense immersion $U \rightarrow V$. Flasque sheaves are flabby in the sense of Definition A.9.4 (see [Rio02, lemme 1.40]).

(2) We say $F \in \underline{\mathbf{M N S}}^{\text {fin }}$ is flasque if $F_{M}$ is flasque for any $M \in \underline{\mathbf{M S m}}^{\text {fin }}$ (see Notation 3.6.1). Again, flasque sheaves are flabby by (3.6.1).

Lemma 3.6.3. Let $I \in \underline{\mathrm{MNST}}^{\mathrm{fin}}$ be an injective object. Then $\underline{\underline{c}}^{\mathrm{finNis}}(I) \in \underline{\mathrm{MNS}}^{\mathrm{fin}}$ is flasque, and hence flabby. 


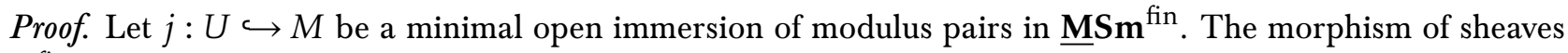
$\mathbb{Z}_{\mathrm{tr}}^{\mathrm{fin}}(j)$ is a monomorphism, hence $j^{*}: I(M) \rightarrow I(U)$ is surjective. Alternatively, one can apply Lemma A.9.5 with (3.5.3) to show that $\underline{c}^{\text {fin } \sigma}(I)$ is flabby. (This proof also works for the étale topology.)

\section{Sheaves on $\underline{M S m}$ and $\underline{M}$ Cor}

\subsection{A cd-structure on $\underline{\mathrm{MSm}}$}

Let $P_{\underline{\mathrm{MV}}}$ be the collection of commutative squares in $\underline{\mathbf{M S m}}$ which are isomorphic in $\underline{\mathbf{M S m}} \mathbf{m}^{\mathrm{Sq}}$ to $\underline{b}_{s}^{\mathrm{Sq}}(Q)$ for some $\underline{\mathrm{MV}}^{\text {fin }}$-square $Q$ in Definition 3.2.1. Then $P_{\underline{\mathrm{MV}}}$ defines a cd-structure on $\underline{\mathrm{MSm}}$ (see §3.2).

Definition 4.1.1. The squares which belong to $\underline{P}_{\mathrm{MV}}$ are called $\underline{\mathrm{MV}}$-squares.

Theorem 4.1.2. The cd-structure $P_{\underline{\mathrm{MV}}}$ is strongly complete and strongly regular, in particular complete and regular (see Definitions A.7.1 and A.7.4).

Proof. This follows from Propositions 3.2.3 and A.7.6.

\subsection{Sheaves on $\underline{\mathrm{MSm}}$}

Definition 4.2.1. Consider the Grothendieck topology on $\underline{\mathrm{MS} m}$ generated by the squares in $P_{\mathrm{MV}}$. The resulting site will be denoted by $\underline{\mathbf{M S}} \mathbf{m}_{\mathrm{Nis}}$. We write $\underline{\mathbf{M N S}}$ for the full subcategory of sheaves in $\underline{\mathrm{MPS}}$. We

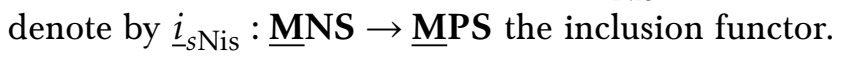

By the general properties of Grothendieck topologies [SGA4, exposé2], we have:

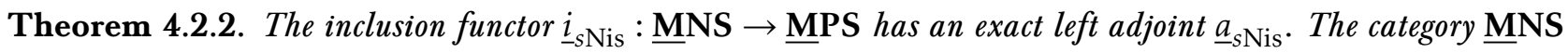
is Grothendieck ( $\$ A .10)$.

Lemma 4.2.3. The following conditions are equivalent for $F \in \underline{\text { MPS. }}$

(i) $F \in \underline{\text { MNS. }}$

(ii) $\underline{b}_{S}^{*} F \in \underline{\mathbf{M N S}}{ }^{\mathrm{fin}}$; in other words, $\left.\underline{b}_{S}^{*} F\right)_{M}$ is a Nisnevich sheaf for any $M \in \underline{\mathbf{M S m}}$ (see (1.9.2) for $\underline{b}_{S}$ and Notation 3.6.1 for $\left.(-)_{M}\right)$.

(iii) F transforms any $\underline{\mathrm{MV}}^{\mathrm{fin}}$-square

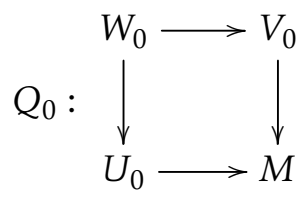

into an exact sequence

$$
0 \rightarrow F(M) \rightarrow F\left(U_{0}\right) \times F\left(V_{0}\right) \rightarrow F\left(W_{0}\right) .
$$

Proof. In view of Theorem 4.1.2 and [Voe10a, Corollary 2.17], we have (i) $\Longleftrightarrow$ (iii). On the other hand, (ii) $\Longleftrightarrow$ (iii) by adjunction and Proposition 3.2.3.

Corollary 4.2.4. The category $\underline{\mathrm{MNS}}$ is closed under infinite direct sums in $\underline{\mathrm{MPS}}$ and $\underline{i}_{-\mathrm{N} \text { is }}$ is strongly additive (Definition 3.3.3).

Proof. This follows from Lemmas 3.3.4, 4.2.3 ((i) $\Longleftrightarrow\left(\right.$ ii)) and A.8.1 (2) because $\underline{b}_{s}^{*}$ is strongly additive as a left adjoint. 


\subsection{The adjunction $\left(\underline{b}_{s, \mathrm{Nis}}, \underline{b}_{s}^{\mathrm{Nis}}\right)$}

Definition 4.3.1. A map in $\underline{\mathbf{M S}} \mathbf{m}_{\mathrm{Nis}}$ is called a strict Nisnevich cover if it is the image of a cover of $\underline{\mathbf{M S}} \mathbf{m}_{\mathrm{Nis}}^{\text {fin }}$ by $\underline{b}_{S}: \underline{\mathbf{M S m}}{ }^{\text {fin }} \rightarrow \underline{\mathbf{M S}}$.

By definition, a strict Nisnevich cover is evidently a cover in $\underline{\mathbf{M S m}}_{\mathrm{Nis}}$. Up to isomorphism, any cover of $\underline{\mathbf{M S m}}_{\mathrm{Nis}}$ can be refined to such a cover. More precisely, we have the following lemma.

Lemma 4.3.2. Any cover $U \rightarrow M$ in $\underline{\mathbf{M S m}}_{\mathrm{Nis}}$ admits a refinement of the form $V \rightarrow N \rightarrow M$, where $V \rightarrow N$ is a strict Nisnevich cover and $N \rightarrow M$ is a morphism in $\underline{\Sigma}^{\text {fin }}$ (see Definition 1.9.1).

Proof. By Definition 4.2.1 and Proposition 1.9.2, there is a refinement of $U \rightarrow M$ of the form

$$
U_{n} \stackrel{f_{n}}{\rightarrow} U_{n-1} \stackrel{f_{n-1}}{\rightarrow} \cdots \stackrel{f_{1}}{\rightarrow} U_{0}=M,
$$

where for each $i$ we have either (i) $f_{i} \in \underline{\Sigma}^{\text {fin }}$, (ii) $f_{i}=g^{-1}$ for some $g \in \underline{\Sigma}^{\text {fin }}$, or (iii) $f_{i}$ is a strict Nisnevich cover. We proceed by induction on $n$, the case $n=0$ being trivial. Suppose $n>0$. By induction, we have a refinement of $U_{n} \rightarrow U_{1}$ of the form $V^{\prime} \rightarrow N^{\prime} \rightarrow U_{1}$ where $V^{\prime} \rightarrow N^{\prime}$ is a strict Nisnevich cover and $N^{\prime} \rightarrow U_{1}$ is in $\underline{\Sigma}^{\text {fin }}$.

If $\bar{f}_{1} \in \underline{\Sigma}^{\text {fin }}$, then we can take $V=V^{\prime}$ and $N=N^{\prime}$, as the composition $N^{\prime} \rightarrow U_{1} \rightarrow U_{0}$ belongs to $\underline{\Sigma}^{\text {fin }}$. Next, suppose $f_{1}=g^{-1}$ with $g \in \underline{\Sigma}^{\mathrm{fin}}$. Then we can take $V=V^{\prime} \times_{U_{1}} U_{0}$ and $N=N^{\prime} \times_{U_{1}} U_{0}$, where $\bar{U}_{0}$ is regarded as a $U_{1}$-scheme by $g$. Finally, suppose $f_{1}$ is a strict Nisnevich cover. By Lemma 1.6.3, we may find a morphism $N \rightarrow U_{0}$ in $\underline{\Sigma}^{\text {fin }}$ such that $N^{\prime \prime}:=N \times_{U_{0}} U_{1} \rightarrow U_{1}$ factors through $N^{\prime}$. Then we can take $V=V^{\prime} \times_{N^{\prime}} N^{\prime \prime}$. This completes the proof.

We define $\underline{b}_{s}^{\text {Nis }}: \underline{\text { MNS }} \rightarrow \underline{\text { MNS }}{ }^{\text {fin }}$ to be the restriction of $\underline{b}_{s}^{*}$, cf. Lemma 4.2 .3 (ii). By definition, we have

$$
\underline{b}_{s}^{*} \underline{i}_{s \mathrm{Nis}}=\underline{i}_{s \mathrm{Nis}}^{\mathrm{fin}} \underline{\underline{b}}_{s}^{\mathrm{Nis}} \text {. }
$$

Proposition 4.3.3. The following assertions hold.

(1) We have $\underline{b}_{s !}\left(\underline{\mathrm{MNS}}^{\mathrm{fin}}\right) \subset \underline{\mathrm{MNS}}$. In particular, $\underline{b}_{s}$ r restricts to $\underline{b}_{s \mathrm{Nis}}: \underline{\mathrm{MNS}} \underline{\mathrm{fin}}^{\rightarrow} \rightarrow \underline{\mathrm{MNS}}$ so that we have

$$
\underline{b}_{s !} \underline{i}_{s \mathrm{Nis}}^{\mathrm{fin}}=\underline{i}_{s \mathrm{Nis}} \underline{b}_{s \mathrm{Nis}} .
$$

(2) The functor $\underline{b}_{s \mathrm{Nis}}$ is an exact left adjoint of $\underline{b}_{s}^{\mathrm{Nis}}$. The functor $\underline{b}_{s}^{\mathrm{Nis}}$ is fully faithful and preserves injectives. The counit map $\underline{b}_{s \mathrm{Nis}} \underline{b}_{s}^{\mathrm{Nis}} \rightarrow$ Id is an isomorphism and $\underline{b}_{s \mathrm{Nis}} \underline{R}^{q} \underline{b}_{s}^{\mathrm{Nis}}=0$ for $q>0$.

Proof. Let $F \in \underline{\mathbf{M N S}} \mathbf{S}^{\text {fin }}$ and take $M \in \underline{\mathbf{M S m}}$. We shall show that $\left(\underline{b}_{s}^{*} \underline{b}_{s !} F\right)_{M}$ is a Nisnevich sheaf on $\bar{M}$. For a given $\underline{M V}^{\text {fin }}$-square in $\underline{\mathbf{M S m}}^{\text {fin }}$

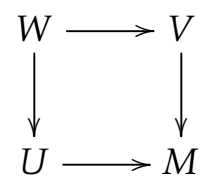

its pullback via $(N \rightarrow M) \in \underline{\Sigma}^{\text {fin }} \downarrow M$ (which exists by Corollary 1.10 .7 (1))

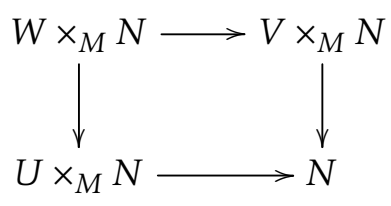

is also an $\underline{M V}^{\text {fin }}$-square. By Proposition 3.2.3 (2) and by [Voel0a, Corollary 2.17], the sequence

$$
0 \rightarrow F(N) \rightarrow F\left(U \times_{M} N\right) \oplus F\left(V \times_{M} N\right) \rightarrow F\left(W \times_{M} N\right)
$$


is exact. By Lemma 1.6.3, the pullback of $\underline{\Sigma}^{\text {fin }} \downarrow M$ via $U \rightarrow M$ is cofinal in $\underline{\Sigma}^{\text {fin }} \downarrow U$, and similarly for $V \rightarrow M$ and $W \rightarrow M$. Hence, by taking its colimit over $N \in \underline{\Sigma}^{\text {fin }} \downarrow M$, the above exact sequences and (2.5.1) imply the desired exact sequence

$$
0 \rightarrow b_{s !} F(M) \rightarrow b_{s !} F(U) \oplus b_{s !} F(V) \rightarrow b_{s !} F(W) .
$$

In view of Lemma 4.2.3, this finishes the proof of (1).

(2) The adjunction $\left(\underline{b}_{s \mathrm{Nis}}, \underline{b}_{s}^{\mathrm{Nis}}\right)$ follows from the adjunction $\left(\underline{b}_{s !}, \underline{b}_{s}^{*}\right)$ (see Proposition 2.5.1), by the full faithfullness of $\underline{i}_{s \mathrm{Nis}}$ and $\underline{i}_{s \mathrm{Nis}}^{\text {fin }}$, and by the formulas (4.3.1) and (4.3.2). The full faithfulness of $\underline{b}_{s}^{\text {Nis }}$ follows from that of $\underline{b}_{s}^{*}$ (see Proposition 2.5.1), $\underline{i}_{s \mathrm{Nis}}$ and $\underline{i}_{s \mathrm{Nis}}^{\mathrm{fin}}$. Then the counit map $\underline{b}_{s \mathrm{Nis}} \underline{b}_{s}^{\mathrm{Nis}} \rightarrow$ Id is an isomorphism by Lemma A.3.1.

We prove the exactness of $\underline{b}_{s \mathrm{Nis}}$ as follows. Since it is right exact as a left adjoint, it suffices to show its left exactness.

Assume given an exact sequence in $\underline{\mathbf{M N S}}{ }^{\mathrm{fin}}$ :

$$
0 \rightarrow F \rightarrow G \rightarrow H \rightarrow 0
$$

Applying the left exact functor $\underline{i}_{s N \text { is }}^{\text {fin }}: \underline{\text { MNS }}^{\text {fin }} \rightarrow \underline{\text { MPS }}^{\text {fin }}$ and the exact functor $\underline{b}_{s}: \underline{\text { MPS }}{ }^{\text {fin }} \rightarrow \underline{\text { MPS }}$ and using (4.3.2), we get an exact sequence

$$
0 \rightarrow \underline{i}_{s \mathrm{Nis}} \underline{b}_{s \mathrm{Nis}} F \rightarrow \underline{i}_{s \mathrm{Nis}} \underline{b}_{s \mathrm{Nis}} G \rightarrow \underline{i}_{s \mathrm{Nis}} \underline{b}_{s \mathrm{Nis}} H .
$$

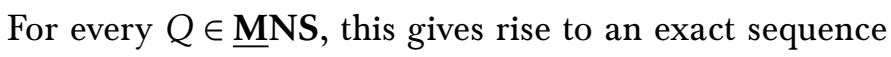

$$
\begin{aligned}
& 0 \rightarrow \operatorname{Hom} \underline{\mathbf{M P S}}\left(\underline{i}_{s \mathrm{Nis}} Q, \underline{i}_{s \mathrm{Nis}} \underline{b}_{s \mathrm{Nis}} F\right) \rightarrow \operatorname{Hom} \underline{\mathrm{MPS}}\left(\underline{i}_{s \mathrm{Nis}} Q, \underline{i}_{s \mathrm{Nis}} \underline{b}_{s \mathrm{Nis}} G\right) \\
& \rightarrow \operatorname{Hom} \underline{\mathbf{M P S}}\left(\underline{i}_{s \mathrm{Nis}} Q, \underline{i}_{s \mathrm{Nis}} \underline{b}_{s \mathrm{Nis}} H\right) \text {. }
\end{aligned}
$$

Since $\underline{i}_{s \text { Nis }}$ is fully faithful, this gives an exact sequence

$$
0 \rightarrow \operatorname{Hom}_{\underline{\mathbf{M N S}}}\left(Q, \underline{b}_{s \mathrm{Nis}} F\right) \rightarrow \operatorname{Hom}_{\underline{\mathbf{M N S}}}\left(Q, \underline{b}_{s \mathrm{Nis}} G\right) \rightarrow \operatorname{Hom}_{\underline{\mathbf{M N S}}}\left(Q, \underline{b}_{s \mathrm{Nis}} H\right),
$$

which shows the exactness of

$$
0 \rightarrow \underline{b}_{s \mathrm{Nis}} F \rightarrow \underline{b}_{s \mathrm{Nis}} G \rightarrow \underline{b}_{s \mathrm{Nis}} H
$$

as desired. Therefore, $\underline{b}_{s \mathrm{Nis}}$ is exact.

Then $\underline{b}_{s}^{\text {Nis }}$ preserves injectives since it has an exact left adjoint $\underline{b}_{s \text { Nis }}$. Moreover, applying $R^{q}(q>0)$ to the counit isomorphism $\underline{b}_{s \mathrm{Nis}} \underline{b}_{s}^{\mathrm{Nis}} \rightarrow \mathrm{Id}$, we have

$$
\underline{b}_{s \mathrm{Nis}} R^{q} \underline{b}_{s}^{\mathrm{Nis}} \simeq R^{q}\left(\underline{b}_{s \mathrm{Nis}} \underline{b}_{s}^{\mathrm{Nis}}\right) \simeq R^{q} \mathrm{Id} \simeq 0,
$$

by Example A.9.2 and the exactness of $\underline{b}_{s \text { Nis }}$. This concludes the proof.

Corollary 4.3.4. We have a natural isomorphism $\underline{a}_{s \mathrm{Nis}} \simeq \underline{b}_{s \mathrm{Nis}} \underline{a}_{s \mathrm{Nis}} \underline{b}_{s}^{\mathrm{fin}}$.

Proof. By the uniqueness of left adjoints, it suffices to check that the right hand side is also left adjoint to $\underline{i}_{s \mathrm{Nis}}$. We first apply double adjunction by $\left(\underline{b}_{s \mathrm{Nis}}, \underline{b}_{s}^{\mathrm{Nis}}\right)$ (Proposition $\left.4.3 .3(2)\right)$ and $\left(\underline{a}_{s \mathrm{Nis}}^{\mathrm{fin}} \underline{-}_{s \mathrm{Nis}}^{\mathrm{fin}}\right)$, then use (4.3.1) and the full faithfulness of $\underline{b}_{s}^{*}$ (Proposition 2.5.1).

\subsection{Cohomology in $\underline{\mathrm{MNS}}$}

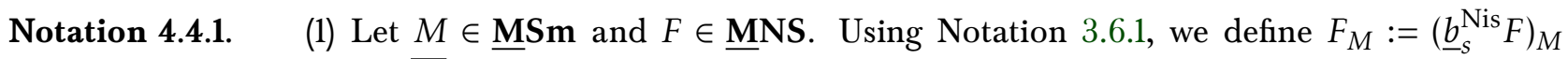
which is a sheaf on $(\bar{M})_{\text {Nis }}$.

(2) For $M \in \underline{\mathbf{M S}} \mathbf{m}$, let $\mathbb{Z}^{p}(M) \in \underline{\text { MPS }}$ be the associated representable additive presheaf (see (2.6.1)) and let

$$
\mathbb{Z}(M)=\underline{a}_{s \mathrm{Nis}} \mathbb{Z}^{p}(M) \in \underline{\mathbf{M N S}}
$$

be the associated sheaf. 
Proposition 4.4.2. For $M \in \underline{\mathrm{MS}}, F \in \underline{\mathrm{MNS}}$ and $i \geq 0$, we have a natural isomorphism

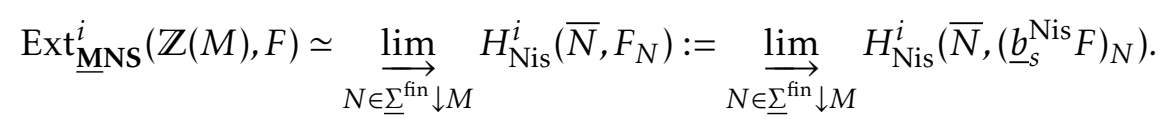

Moreover, we have

$$
\underset{N \in{\underline{\sum^{\mathrm{fin}}} \downarrow M}_{\lim }^{\longrightarrow}}{i} H_{\mathrm{Nis}}^{i}\left(\bar{N},\left(R^{q} \underline{b}_{s}^{\mathrm{Nis}} F\right)_{N}\right)=0 \text { for all } q>0 \text {. }
$$

Proof. Define functors $\Gamma_{M}^{\downarrow}: \underline{\text { MNS }}{ }^{\text {fin }} \rightarrow$ Ab and $\underline{\Gamma}_{M}: \underline{\text { MNS }} \rightarrow$ Ab by

$$
\Gamma_{M}^{\downarrow}(G)=\underset{N \in \underline{\Sigma}^{\mathrm{fin}} \downarrow M}{\lim } G(N), \quad \underline{\Gamma}_{M}(F)=F(M) .
$$

We have $\Gamma_{M}^{\downarrow}=\underline{\Gamma}_{M} \underline{b}_{s \text { Nis }}$. By Theorem A.9.1 and Lemma 4.4.3 below, we get $\left(R^{p} \underline{\Gamma}_{M}\right) \underline{b}_{s \text { Nis }}=R^{p} \Gamma_{M}^{\downarrow}$ for any $p \geq 0$ since $\underline{b}_{s \text { Nis }}$ is exact. Thus, by Lemma 4.4 .4 below we obtain

$$
\operatorname{Ext}_{\underline{\mathbf{M N S}}}^{p}\left(\mathbb{Z}(M), \underline{b}_{s \mathrm{Nis}} G\right) \cong \underset{N \in \underline{\Sigma}^{\mathrm{fin}} \downarrow M}{\underset{\lim }{\longrightarrow}} H_{\mathrm{Nis}}^{p}\left(\bar{N}, G_{N}\right)
$$

for any $G \in \underline{\mathbf{M N S}} \mathbf{S}^{\text {fin }}$ and $p \geq 0$. Setting $G=R^{q} \underline{b}_{s}^{\text {Nis }} F$, we get (4.4.2) for $q=0$ (resp. (4.4.3) for $q>0$ ) thanks to Proposition 4.3.3 (2).

Lemma 4.4.3. For an injective $I \in \underline{\mathrm{MNS}}{ }^{\mathrm{fin}}, \underline{b}_{s \mathrm{Nis}} I \in \underline{\mathrm{MNS}}$ is flabby (see Definition A.9.4).

Proof. Write $F=\underline{b}_{\text {Nis }} I$. By Lemma A.9.3, it suffices to show the vanishing of the canonical map $\check{H}^{q}(U / M, F) \rightarrow \check{H}^{q}(M, F)$ for any cover $U \rightarrow M$ in $\underline{\mathbf{M S m}}_{\mathrm{Nis}}$ and any $q>0$. By Lemma 4.3.2, we may

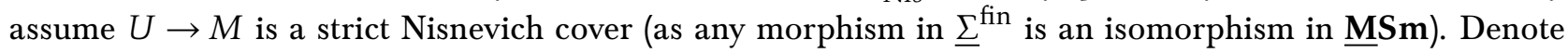
by $U_{M}^{n} \in \underline{\text { MSm }}$ the $n$-fold fiber product of $U$ over $M$ in $\underline{\text { MSm }}$ (which exists by Corollary 1.10 .7 (1)). Then $\check{H}^{q}(U / M, F)$ is computed as the cohomology of the complex whose term in degree $q$ is given by

$$
\underset{L_{q} \in \underline{\lim }^{\mathrm{fin}} \downarrow U_{M}^{q+1}}{\longrightarrow} I\left(L_{q}\right)
$$

By Lemma 1.6.3, for any integer $n>0$ and given $L_{q} \in \underline{\Sigma}^{\text {fin }} \downarrow U_{M}^{q+1}$ for $0 \leq q \leq n$, there exists $L \in \underline{\Sigma}^{\text {fin }} \downarrow M$ in such that $L \times_{M} U_{M}^{q+1} \rightarrow U_{M}^{q+1}$ factor through $L_{q}$ for all $q=0, \ldots, n$. This implies that for $0 \leq q \leq n-1$ the canonical map $\check{H}^{q}\left(U / M, \underline{b}_{s \mathrm{Nis}} I\right) \rightarrow \check{H}^{q}\left(M, \underline{b}_{s \mathrm{Nis}} I\right)$ factors through

$$
\underset{L \in \underline{\lim }^{\mathrm{fin}} \downarrow M}{ } \check{H}^{q}\left(U \times_{M} L / L, I\right),
$$

where $\check{H}^{q}\left(U \times_{M} L / L, I\right)$ is the Čech cohomology of $I$ with respect to the cover $U \times_{M} L \rightarrow L$ in $\underline{\mathbf{M S m}}{ }^{\text {fin }}$, but it vanishes since $I$ is injective in $\underline{\text { MNS }}{ }^{\text {fin }}$. This proves the desired vanishing and completes the proof of Lemma 4.4.3.

Lemma 4.4.4. For any $G \in \underline{\mathbf{M N S}^{\text {fin }}}$ and $p \geq 0$, we have

$$
R^{p} \Gamma_{M}^{\downarrow}(G) \cong \underbrace{\lim }_{N \in \underline{\Sigma}^{\mathrm{fin}} \downarrow M} H_{\mathrm{Nis}}^{p}\left(\bar{N}, G_{N}\right) .
$$

Proof. Take an injective resolution $G \rightarrow I^{\bullet}$ in $\underline{\text { MNS }}{ }^{\text {fin }}$. Then we have

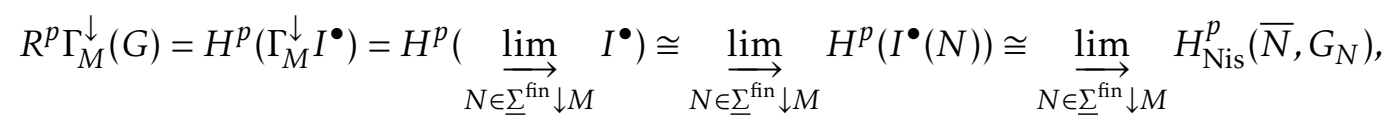

where we used Corollary 1.9.3 for the last-but-one isomorphism, and (3.6.1) for the last one. 


\subsection{Sheaves on MCor}

Lemma 4.5.1. For $F \in \underline{\mathrm{MPST}}$, one has $\underline{c}^{*} F \in \underline{\mathrm{MNS}}$ if and only if $b^{*} F \in \underline{\mathbf{M}} \mathbf{N S T}^{\mathrm{fin}}$.

Proof. This follows from (2.6.2) and Definitions 3.5.1 and 4.2.1.

Definition 4.5.2. We define $\underline{\text { MNST }}$ to be the full subcategory of $\underline{\text { MPST }}$ consisting of those $F$ enjoying the conditions of Lemma 4.5.1. We denote by $\underline{i}_{\mathrm{Nis}}: \underline{\mathrm{MNST}} \rightarrow \underline{\text { MPST }}$ the inclusion functor.

Lemma 4.5.3. The category $\underline{\mathrm{MNST}}$ is closed under infinite direct sums in $\underline{\mathrm{MPST}}$, and $\underline{\underline{\mathrm{N}}}_{\mathrm{Nis}}$ is strongly additive (Definition 3.3.3). It contains $\mathbb{Z}_{\mathrm{tr}}(M)$ for any $M \in \underline{\text { MCor. }}$

Proof. This follows from Lemma 3.5.2, because $\underline{b}^{*}$ is strongly additive as a left adjoint. The last statement follows from Lemma 3.5.2.

By Definition 4.5.2 and Lemma 4.5.1, the functors $\underline{b}^{*}$ and $\underline{c}^{*}$ restrict to $\underline{b}^{\mathrm{Nis}}: \underline{\mathrm{MNST}} \rightarrow \underline{\mathrm{MNST}}$ fin and $\underline{c}^{\mathrm{Nis}}: \underline{\text { MNST }} \rightarrow \underline{\text { MNS. It holds that }}$

$$
\begin{aligned}
& \underline{b}^{*} \underline{i}_{\mathrm{Nis}}=\underline{i}_{\mathrm{Nis}}^{\mathrm{fin}} \underline{\underline{b}}^{\mathrm{Nis},}, \quad \underline{c}^{*} \underline{i}_{\mathrm{Nis}}=\underline{i}_{s \mathrm{Nis}} \underline{c}^{\mathrm{Nis}}, \\
& \underline{b}_{s}^{\mathrm{Nis}} \underline{c}^{\mathrm{Nis}}=\underline{c}^{\mathrm{finNis}} \underline{\underline{N}}^{\mathrm{Nis}}, \quad \underline{b}_{s \mathrm{Nis}} \underline{c}^{\mathrm{finNis}}=\underline{c}^{\mathrm{Nis}} \underline{b}_{\mathrm{Nis}},
\end{aligned}
$$

where for the last two formulas we used (2.6.2).

Proposition 4.5.4. The following assertions hold.

(1) We have $\underline{b}_{!}\left(\underline{\mathrm{MNST}}^{\mathrm{fin}}\right) \subset \underline{\mathrm{MNST}}$.

(2) Let $\underline{b}_{\mathrm{Nis}}: \underline{\mathrm{MNST}}^{\mathrm{fin}} \rightarrow \underline{\mathrm{MNST}}$ be the restriction of $\underline{b}_{\text {! }}$ so that we have

$$
\underline{b}_{!} \underline{i}_{\mathrm{Nis}}^{\mathrm{fin}}=\underline{i}_{\mathrm{Nis}} \underline{b}_{\mathrm{Nis}} \text {. }
$$

Then, the functor $\underline{b}_{\mathrm{Nis}}$ is an exact left adjoint of $\underline{b}^{\mathrm{Nis}}$, which is fully faithful.

(3) The functor $\underline{b}^{\mathrm{Nis}}$ preserves injectives.

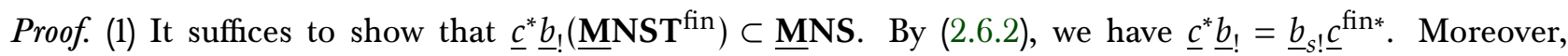
$\underline{c}^{\text {fin* }}{ }^{\text {MNST }}{ }^{\text {fin }} \subset \underline{\text { MNS }}{ }^{\text {fin }}$ by Definition 3.5.1 and $\underline{b}_{s !} \underline{\mathbf{M}} \underline{\mathbf{P S}}^{\text {fin }} \subset \underline{\text { MNS by Proposition }} 4.3 .3$ (1). In (2), the adjointness and the full faithfulness are seen by using Proposition 2.5.1, (4.5.1) and (4.5.2). This proves that $\underline{b}_{\mathrm{Nis}}$ is right exact, and it is also exact by (4.5.2) and Proposition 2.5.1 (see also the proof of the exactness of $\underline{b}_{s \mathrm{Nis}}$ in Proposition 4.3.3 (2)). (3) is a consequence of (2).

Theorem 4.5.5. The inclusion functor $\underline{i}_{\mathrm{Nis}}: \underline{\mathrm{MNST}} \rightarrow \underline{\text { MPST }}$ has the exact left adjoint $\underline{a}_{\mathrm{Nis}}=\underline{b}_{\mathrm{Nis}} \underline{a}_{\mathrm{Nis}} \underline{b}^{\mathrm{fin}}$. In

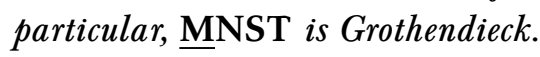

Proof. The formula defining $\underline{a}_{\mathrm{Nis}}$ yields a left adjoint to $\underline{i}_{\mathrm{Nis}}$ by the full faithfulness of $\underline{b}^{*}$ (Proposition 2.5.1) and the adjunctions $\left(\underline{a}_{\mathrm{Nis}}^{\mathrm{fin}}, \underline{\mathrm{i}}_{\mathrm{Nis}}^{\mathrm{fin}}\right)$ and $\left(\underline{b}_{\mathrm{Nis}}, \underline{b}^{\mathrm{Nis}}\right)$ (use (4.5.1)). Its exactness follows from the exactness of the three functors.

Proposition 4.5.6. We have

$$
\underline{b}_{\mathrm{Nis}} \underline{a}_{\mathrm{Nis}}^{\mathrm{fin}}=\underline{a}_{\mathrm{Nis}} \underline{b}_{!}, \quad \underline{a}_{S \mathrm{Nis}} \underline{c}^{*}=\underline{c}^{\mathrm{Nis}} \underline{a}_{\mathrm{Nis}} .
$$

Moreover, $\underline{c}^{\mathrm{Nis}}$ is faithful, exact, strongly additive (Definition 3.3.3) and has a left adjoint $\underline{\underline{c}}_{\mathrm{Nis}}=\underline{a}_{\mathrm{Nis}} \underline{\underline{c}}_{\underline{s} \mathrm{Nis}}$ such that $\underline{c}_{\mathrm{Nis}} \underline{a}_{s \mathrm{Nis}}=\underline{a}_{\mathrm{Nis}} \underline{c}_{!}$.

Proof. The first equality follows from the first formula of (4.5.1) by adjunction. For the second, we use Theorems 4.2.2 and 4.5.5, together with (2.6.2), (3.5.2) and (4.5.1). The last statement follows from Lemma A.8.1 (3). 
Theorem 4.5.7. If $p: U \rightarrow M$ is a cover in $\underline{\mathrm{MSm}}_{\mathrm{Nis}}$, then the Cech complex

$$
\cdots \rightarrow \mathbb{Z}_{\mathrm{tr}}\left(U \times_{M} U\right) \rightarrow \mathbb{Z}_{\mathrm{tr}}(U) \rightarrow \mathbb{Z}_{\mathrm{tr}}(M) \rightarrow 0
$$

is exact in $\underline{\mathrm{MNST}}$. (Note that the fiber products exist in $\underline{\mathrm{MSm}}$ by Corollary 1.10 .7 (1).) Moreover, the sequence

$$
0 \rightarrow \mathbb{Z}_{\mathrm{tr}}(W) \rightarrow \mathbb{Z}_{\mathrm{tr}}(U) \oplus \mathbb{Z}_{\mathrm{tr}}(V) \rightarrow \mathbb{Z}_{\mathrm{tr}}(X) \rightarrow 0
$$

is exact in $\underline{\mathrm{MNST}}$ for any $\underline{\mathrm{MV}}^{\mathrm{fin}}$-square (3.2.1) in $\underline{\mathrm{MSm}}^{\text {fin }}$.

Proof. By Lemma 4.3.2, we may assume $M \rightarrow U$ is a strict Nisnevich cover. Then, by (3.5.3) the complex

$$
\cdots \rightarrow \mathbb{Z}_{\mathrm{tr}}^{\mathrm{fin}}\left(U \times_{M} U\right) \rightarrow \mathbb{Z}_{\mathrm{tr}}^{\mathrm{fin}}(U) \rightarrow \mathbb{Z}_{\mathrm{tr}}^{\mathrm{fin}}(M) \rightarrow 0
$$

is exact in $\underline{\mathrm{MNST}}^{\text {fin }}$. Applying the exact functor $\underline{b}_{\mathrm{Nis}}$, we get (4.5.4). The second statement follows from the first and a small computation (cf. [MVW06, Proposition 6.14]).

\subsection{Cohomology in $\underline{\mathrm{MNST}}$}

Lemma 4.6.1. Let $I \in \underline{\mathrm{MNST}}$ be an injective object. Then $\underline{c}^{\mathrm{Nis}}(I) \in \underline{\mathrm{MNS}}$ is flabby.

Proof. This follows from Lemma A.9.5 and Theorem 4.5.7.

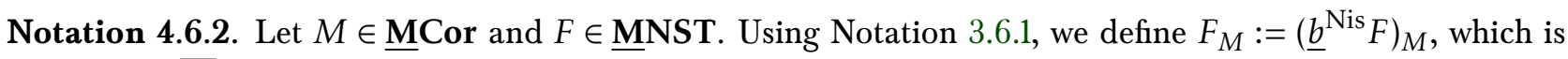
a sheaf on $(\bar{M})_{\text {Nis }}$.

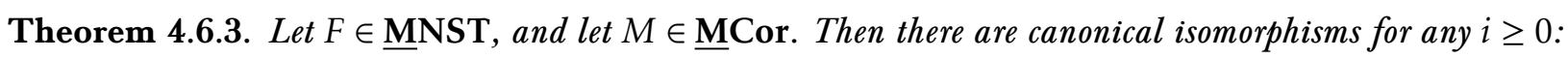

$$
\operatorname{Ext}_{\underline{\mathbf{M N S T}}}^{i}\left(\mathbb{Z}_{\mathrm{tr}}(M), F\right) \simeq \operatorname{Ext}_{\underline{\mathbf{M N S}}}^{i}\left(\mathbb{Z}(M), \underline{\underline{N}}^{\mathrm{Nis}} F\right) \simeq \underset{N \in \underline{\underline{\Sigma}}^{\mathrm{fin}} \downarrow M}{\lim } H_{\mathrm{Nis}}^{i}\left(\bar{N}, F_{N}\right) .
$$

(See (4.4.1) for $\mathbb{Z}(M)$.) Moreover, we have

$$
\underset{N \in \underline{\underline{\Sigma}}_{\mathrm{fin}}^{\lim } \downarrow M}{ } H_{\mathrm{Nis}}^{i}\left(\bar{N},\left(R^{q}\left(\underline{b}_{s}^{\mathrm{Nis}}\right) \underline{\underline{N}}^{\mathrm{Nis}} F\right)_{N}\right)=0 \text { for all } q>0 .
$$

Proof. Applying the last identity of Proposition 4.5 .6 to $\mathbb{Z}^{p}(M)$, we get

$$
\underline{c}_{\mathrm{Nis}} \mathbb{Z}(M)=\underline{a}_{\mathrm{Nis}} \underline{c}_{!} \mathbb{Z}^{p}(M)=\underline{a}_{\mathrm{Nis}} \mathbb{Z}_{\mathrm{tr}}(M)=\mathbb{Z}_{\mathrm{tr}}(M)
$$

where the second equality follows from (2.6.1), and the third one holds by Lemma 4.5.3. This yields an isomorphism

$$
\underline{\operatorname{MNS}}\left(\mathbb{Z}(M), \underline{c}^{\mathrm{Nis}} F\right) \simeq \underline{\operatorname{MNST}}\left(\mathbb{Z}_{\mathrm{tr}}(M), F\right)
$$

which is the case $i=0$ of the first isomorphism in the proposition. The general case $i \geq 0$ then follows from Theorem A.9.1, Lemma 4.6.1 and the exactness of $\underline{c}^{\text {Nis }}$ (Proposition 4.5.6), and the second isomorphism follows from Proposition 4.4.2 and (4.5.1). The last assertion follows from (4.4.3).

\section{A. Categorical toolbox, I}

This appendix gathers known and less-known results that we use constantly. 
A.1. Pro-objects ([SGA4, exposé I, §8], [AM69, Appendix 2])

Recall that a pro-object of a category $\mathcal{C}$ is a functor $F: A \rightarrow \mathcal{C}$, where $A$ is a small cofiltered category (dual of [Mac98, Chapter IX, §1]). They are denoted by $\left\{X_{\alpha}\right\}_{\alpha \in A}$ or by “ $\lim ^{\longleftarrow}{ }_{\alpha \in A} X_{\alpha}$ (Deligne's notation), with $X_{\alpha}=F(\alpha)$. Pro-objects of $\mathcal{C}$ form a category pro-C , with morphisms given by the formula

$$
\text { pro- } \mathcal{C}\left(\left\{X_{\alpha}\right\}_{\alpha \in A},\left\{Y_{\beta}\right\}_{\beta \in B}\right)=\underset{\beta \in B}{\lim } \underset{\alpha \in A}{\lim } \mathcal{C}\left(X_{\alpha}, Y_{\beta}\right)
$$

There is a canonical full embedding $c: \mathcal{C} \hookrightarrow$ pro- $\mathcal{C}$, sending an object to the corresponding constant pro-object $(A=\{*\})$.

For the next lemma, we recall a special case of comma categories from Mac Lane [Mac98, Chapter II, §6]. If $\psi: \mathcal{A} \rightarrow \mathcal{B}$ is a functor and $b \in \mathcal{B}$, we write $b \downarrow \psi$ for the category whose objects are pairs $(a, f) \in \mathcal{A} \times \mathcal{B}(b, \psi(a))$; a morphism $\left(a_{1}, f_{1}\right) \rightarrow\left(a_{2}, f_{2}\right)$ is a morphism $g \in \mathcal{A}\left(a_{1}, a_{2}\right)$ such that $f_{2}=\psi(g) f_{1}$. The category $\psi \downarrow b$ is defined dually (objects: systems $\psi(a) \stackrel{f}{\rightarrow} b$, etc.) According to [Mac98, Chapter IX, $\S 3], \psi$ is final if, for any $b \in \mathcal{B}$, the category $\psi \downarrow b$ is nonempty and connected; here we shall use the dual property cofinal (same conditions for $b \downarrow \psi$ ). As usual, we abbreviate $I d_{\mathcal{A}} \downarrow a$ and $a \downarrow I d_{\mathcal{A}}$ by $\mathcal{A} \downarrow a$ and $a \downarrow \mathcal{A}$.

Let $F=(F: A \rightarrow \mathcal{C})=\left\{X_{\alpha}\right\}_{\alpha \in A} \in$ pro-C . For each $\alpha \in A$, we have a "projection" morphism $\pi_{\alpha}: F \rightarrow c\left(X_{\alpha}\right)$ in pro- $\mathcal{C}$. This yields an isomorphism in pro- $\mathcal{C}$

$$
F \stackrel{\sim}{\rightarrow} \lim _{\alpha \in A} c\left(X_{\alpha}\right)
$$

(explaining Deligne's notation) and a functor

$$
\theta: A \rightarrow F \downarrow c, \quad \theta(\alpha)=\left(X_{\alpha}, \pi_{\alpha}\right),
$$

where we take $\mathcal{A}=\mathcal{C}$ and $\mathcal{B}=$ pro $-\mathcal{C}$ in the above setting.

Lemma A.1.1. The functor $\theta$ is cofinal.

Proof. Let $F \stackrel{f}{\rightarrow} c(Y)(Y \in \mathcal{C})$ be an object of $F \downarrow c$. An object of $\theta \downarrow(F \stackrel{f}{\rightarrow} c(Y))$ is a pair $(\alpha, \varphi)$, with $\alpha \in A$ and $\varphi: F(\alpha) \rightarrow Y$ such that $f=c(\varphi) \pi_{\alpha}$. This category is nonempty because an object $\alpha \in A$ and the morphism $f$ yield the object $f(\alpha): F(\alpha) \rightarrow c(Y)(\alpha)=Y$, and we have $(\alpha, f(\alpha)) \in \theta \downarrow(F \stackrel{f}{\rightarrow} c(Y))$. Note also that it is cofiltered, because $A$ is. Since any cofiltered category is obviously connected, we are done.

(Warning: the use of co in (co)final and (co)filtered is opposite in [Mac98] and in [KS06]. We use the convention of [Mac98].)

\section{A.2. Pro-adjoints [SGA4, exposé I, §8.11.5]}

Let $u: \mathcal{C} \rightarrow \mathcal{D}$ be a functor: it induces a functor pro- $u:$ pro- $\mathcal{C} \rightarrow$ pro- $\mathcal{D}$.

Recall standard terminology for the functoriality of limits (=inverse limits) and colimits (= direct limits):

Definition A.2.1. A functor $u: \mathcal{C} \rightarrow \mathcal{D}$ is left exact (resp. right exact, resp. exact) if it commutes with finite limits (resp. finite colimits, resp. finite limits and colimits).

Proposition A.2.2 (dual of [SGA4, exposé I, proposition 8.11.4]). Consider the following conditions:

(i) The functor pro-u has a left adjoint.

(ii) There exists a functor $v: \mathcal{D} \rightarrow$ pro- $\mathcal{C}$ and an isomorphism

$$
\text { pro- } \mathcal{C}(v(d), c) \simeq \mathcal{D}(d, u(c))
$$

contravariant in $d \in \mathcal{D}$ and covariant in $c \in \mathcal{C}$.

(iii) $u$ is left exact. 
Then (i) $\Longleftrightarrow$ (ii) $\Rightarrow$ (iii), and (iii) $\Rightarrow$ (i) ifC is essentially small and closed under finite inverse limits.

(The condition on finite inverse limits appears in [AM69, p. 158], but is skipped in [SGA4, exposé I, proposition 8.11.4].)

Definition A.2.3. In Condition (ii) of Proposition A.2.2, we say that $v$ is pro-left adjoint to $u$.

\section{A.3. Localisation ([GZ67, Chapter I], see also [KS06, Chapter 7])}

Let $\mathcal{C}$ be a category, and let $\Sigma \subset A r(\mathcal{C})$ be a class of morphisms: following Grothendieck and Maltsiniotis, we call $(\mathcal{C}, \Sigma)$ a localiser. Consider the functors $F: \mathcal{C} \rightarrow \mathcal{D}$ such that $F(s)$ is invertible for all $s \in \Sigma$. This "2-universal problem" has a solution $Q: \mathcal{C} \rightarrow \mathcal{C}\left[\Sigma^{-1}\right]$. One may choose $\mathcal{C}\left[\Sigma^{-1}\right]$ to have the same objects as $\mathcal{C}$ and $Q$ to be the identity on objects; then $\mathcal{C}\left[\Sigma^{-1}\right]$ is unique (not just up to unique equivalence of categories). If $\mathcal{C}$ is essentially small, then $\mathcal{C}\left[\Sigma^{-1}\right]$ is small, but in general the sets $\mathcal{C}\left[\Sigma^{-1}\right](X, Y)$ may be "large"; one can sometimes show that it is not the case (Corollary A.5.4). A functor of the form $Q: \mathcal{C} \rightarrow \mathcal{C}\left[\Sigma^{-1}\right]$ will be called a localisation. We have a basic result on adjoint functors [GZ67, Chapter I, Proposition 1.3]:

Lemma A.3.1. Let $G: \mathcal{C} \leftrightarrows \mathcal{D}: D$ be a pair of adjoint functors ( $G$ is left adjoint to $D$ ). Then the following conditions are equivalent:

(i) D is fully faithful.

(ii) The counit $G D \Rightarrow I d_{\mathcal{D}}$ is a natural isomorphism.

(iii) $G$ is a localisation.

The same holds if $G$ is right adjoint to $D$ (replacing the counit by the unit).

Definition A.3.2. Let $(\mathcal{C}, \Sigma)$ be a localiser, and let $Q: \mathcal{C} \rightarrow \mathcal{C}\left[\Sigma^{-1}\right]$ be the corresponding localisation functor. We write

$$
\operatorname{sat}(\Sigma)=\{s \in \operatorname{Ar}(\mathcal{C}) \mid Q(s) \text { is invertible }\} .
$$

This is the saturation of $\Sigma$; we say that $\Sigma$ is saturated if $\operatorname{sat}(\Sigma)=\Sigma$.

Lemma A.3.3 ([GZ67, Chapter I, Lemma 1.2]). Let $(\mathcal{C}, \Sigma)$ be a localiser, $\mathcal{D}$ a category, $F, G: \mathcal{C}\left[\Sigma^{-1}\right] \rightarrow \mathcal{D}$ two functors and $u: F \circ Q \Rightarrow G \circ Q$ a natural transformation, where $Q: \mathcal{C} \rightarrow \mathcal{C}\left[\Sigma^{-1}\right]$ is the localisation functor. Then $u$ induces a unique natural transformation $\bar{u}: F \Rightarrow G$.

Proof. Define $\bar{u}_{X}=u_{X}: F(X) \rightarrow G(X)$ for $X \in O b \mathcal{C}\left[\Sigma^{-1}\right]=O b \mathcal{C}$. We must show that $\bar{u}$ commutes with the morphisms of $\mathcal{C}\left[\Sigma^{-1}\right]$. This is obvious, since $u$ commutes with the morphisms of $\mathcal{C}$ and the morphisms of $\mathcal{C}\left[\Sigma^{-1}\right]$ are expressed as fractions in the morphisms of $\mathcal{C}$.

\section{A.4. Presheaves and pro-adjoints}

Let $\mathcal{C}$ be a category. We write $\hat{\mathcal{C}}$ for the category of presheaves of sets on $\mathcal{C}$ (i.e. functors $\mathcal{C}^{\text {op }} \rightarrow$ Set); it comes with the Yoneda embedding

$$
y: \mathcal{C} \rightarrow \hat{\mathcal{C}}
$$

which sends an object to the corresponding representable presheaf. If $u: \mathcal{C} \rightarrow \mathcal{D}$ is a functor, we have the standard sequence of three adjoint functors

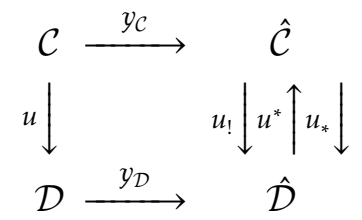

where $u_{\text {! }}$ extends $u$ through the Yoneda embeddings [SGA4, exposé I, proposition 5.4]; $u_{!}$and $u_{*}$ are computed by the usual formulas for left and right Kan extensions (loc. cit., (5.1.1)). If $u$ has a left adjoint $v$, the sequence $\left(u_{!}, u^{*}, u_{*}\right)$ extends to

$$
\left(v_{!}, v^{*}=u_{!}, v_{*}=u^{*}, u_{*}\right)
$$


(ibid., Remark 5.5.2).

Let $\mathcal{A}$ be an essentially small additive category. Instead of presheaves of sets on $\mathcal{A}$, one usually uses the category Mod $-\mathcal{A}$ of additive presheaves of abelian groups; the above results transfer to this context, mutatis mutandis.

\section{Proposition A.4.1.}

a) The functor $u_{!}\left(\right.$resp. $\left.u_{*}, u^{*}\right)$ commutes with all representable colimits (resp. limits, limits and colimits). If $u$ has a left adjoint, then $u_{!}$also commutes with all limits. If $u$ has a pro-left adjoint $v$ (Definition A.2.3), so does $u_{!}$which is therefore exact. Moreover, $u_{!}$is then given by the formula

$$
\left(u_{!} F\right)(Y)=\underline{\lim }(F \circ v(Y)), \quad F \in \hat{\mathcal{C}}, Y \in \mathcal{D} .
$$

b) If $u$ is fully faithful, so is $u_{!}$.

c) If $u$ is a localisation or is full and essentially surjective, then $u_{!}$is a localisation.

d) In the case of c), for $C \in \mathcal{C}$ the following conditions are equivalent:

(i) The representable functor $y_{\mathcal{C}}(C) \in \hat{\mathcal{C}}$ induces a functor on $\mathcal{D}$ via $u$.

(ii) The unit map $y_{\mathcal{C}}(C) \rightarrow u^{*} u_{!} y_{\mathcal{C}}(C) \simeq u^{*} y_{\mathcal{D}}(u(C))$ is an isomorphism.

(iii) For any $C^{\prime} \in \mathcal{C}$, the map $\mathcal{C}\left(C^{\prime}, C\right) \rightarrow \mathcal{D}\left(u\left(C^{\prime}\right), u(C)\right)$ induced by $u$ is bijective.

Proof. a) follows from general properties of adjoint functors, except for the case of a pro-left adjoint. Let $u$ admit a pro-left adjoint $v$, and let $Y \in \mathcal{D}$ : so there is an isomorphism of categories $Y \downarrow u \simeq v(Y) \downarrow c$. Hence, we get by Lemma A.1.1 a cofinal functor

$$
A \rightarrow Y \downarrow u,
$$

where $A$ is the indexing set of $v(Y)$. Thus, for $F \in \hat{\mathcal{C}}, u_{!} F(Y)$ may be computed as

$$
u_{!} F(Y)=\underset{\alpha \in A}{\lim _{\longrightarrow}} F(v(Y)(\alpha))=\text { pro- } \mathcal{C}\left(y_{\mathcal{C}}(v(Y)), c(F)\right) .
$$

The first equality is the formula in the proposition. The second one shows that the pro-left adjoint $v_{\text {! }}$ of $u_{!}$is defined at $y_{\mathcal{D}}(Y)$ by $y_{\mathcal{C}}(v(Y))$; since any object of $\hat{\mathcal{D}}$ is a colimit of representable objects, this shows that $v_{!}$is defined everywhere.

For b), see [SGA4, exposé I, proposition 5.6]. In c), it is equivalent to show that $u^{*}$ is fully faithful by Lemma A.3.1. Let $F, G \in \hat{\mathcal{D}}$, and let $\varphi: u^{*} F \rightarrow u^{*} G$ be a morphism of functors. In both cases, $u$ is essentially surjective: given $X \in \mathcal{D}$ and an isomorphism $\alpha: X \stackrel{\sim}{\rightarrow} u(Y)$, we get a morphism

$$
\psi_{X}: F(X) \stackrel{\alpha^{*-1}}{\longrightarrow} F(u(Y)) \stackrel{\varphi_{Y}}{\longrightarrow} G(u(Y)) \stackrel{\alpha^{*}}{\longrightarrow} G(X) .
$$

The fact that $\psi_{X}$ is independent of $(Y, \alpha)$ and is natural in $X$ is an easy consequence of each hypothesis (see Lemma A.3.3 in the first case).

In d), the equivalence (ii) $\Longleftrightarrow$ (iii) is tautological and (iii) $\Rightarrow$ (i) is obvious. The implication (i) $\Rightarrow$ (iii) was proven in [GZ67, Chapter I, §4.1.2] assuming that $u$ is a localisation enjoying a calculus of left fractions; let us prove (i) $\Rightarrow$ (ii) in general. Under (i), we have $y_{\mathcal{C}}(C) \simeq u^{*} F$ for some $F \in \hat{D}$; the unit map becomes

$$
\eta_{u^{*} F}: u^{*} F \rightarrow u^{*} u_{!} u^{*} F
$$

On the other hand, the counit map $\varepsilon_{F}: u_{!} u^{*} F \rightarrow F$ is invertible by the full faithfulness of $u^{*}$. By the adjunction identities, we have $u^{*}\left(\varepsilon_{F}\right) \circ \eta_{u^{*} F}=1_{u^{*} F}$. Hence the conclusion.

We shall usually write $u^{!}$for the pro-left adjoint of $u_{!}$, when it exists. 


\section{A.5. Calculus of fractions}

Definition A.5.1 (dual of [GZ67, Chapter I, Lemma 1.2]). A localiser $(\mathcal{C}, \Sigma)$ (or simply $\Sigma$ ) enjoys a calculus of right fractions if:

(i) The identities of $\mathcal{C}$ are in $\Sigma$.

(ii) $\sum$ is stable under composition.

(iii) (Ore condition.) For each diagram $X^{\prime} \stackrel{s}{\rightarrow} X \stackrel{u}{\leftarrow} Y$ where $s \in \Sigma$, there exists a commutative square

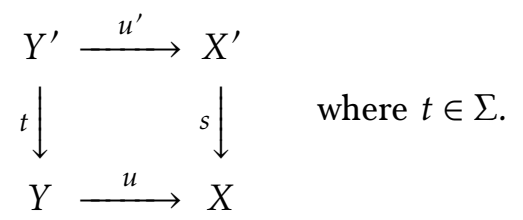

(iv) (Cancellation.) If $f, g: X \rightrightarrows Y$ are morphisms in $\mathcal{C}$ and $s: Y \rightarrow Y^{\prime}$ is a morphism of $\Sigma$ such that $s f=s g$, there exists a morphism $t: X^{\prime} \rightarrow X$ in $\sum$ such that $f t=g t$.

Proposition A.5.2. Suppose that $\sum$ enjoys a calculus of right fractions. For $c \in \mathcal{C}$, let $\Sigma \downarrow c$ denote the full subcategory of the comma category $\mathcal{C} \downarrow c$ given by the objects $c^{\prime} \stackrel{s}{\rightarrow} c$ with $s \in \sum$. Then

a) $\sum \downarrow c$ is cofiltered.

b) [GZ67, Chapter I, 2.3] For any $d \in \mathcal{C}$, the obvious map

$$
\underset{c^{\prime} \in \Sigma \downarrow c}{\underset{\lim _{\longrightarrow}}{\longrightarrow}} \mathcal{C}\left(c^{\prime}, d\right) \rightarrow \mathcal{C}\left[\Sigma^{-1}\right](c, d)
$$

is an isomorphism.

c) Any morphism in $\mathcal{C}\left[\Sigma^{-1}\right]$ is of the form $Q(f) Q(s)^{-1}$ for $f \in A r(\mathcal{C})$ and $s \in \Sigma$; if $f_{1}, f_{2}$ are two parallel arrows in $\mathcal{C}$, then $Q\left(f_{1}\right)=Q\left(f_{2}\right)$ if and only if there exists $s \in \sum$ such that $f_{1} s=f_{2} s$.

Proof. a) We need to check the two conditions (which are dual to those from [Mac98, p. 211]): (1) given two objects $d, d^{\prime} \in \Sigma \downarrow c$, there are arrows $d \leftarrow e \rightarrow d^{\prime}$ in $\Sigma \downarrow c$; (2) given two parallel arrows $f, g: e \rightarrow d$ in $\sum \downarrow c$, there is an arrow $h: e^{\prime} \rightarrow e$ in $\Sigma \downarrow c$ such that $f h=g h$. (1) (resp. (2)) follows from Axioms (iii) and (ii) (resp. (iv) and (ii)) of Definition A.5.1.

b) The "obvious map" (A.5.1) sends a pair $\left(c^{\prime} \stackrel{s}{\rightarrow} c, c^{\prime} \stackrel{f}{\rightarrow} d\right)$ with $s \in \Sigma$ and $f \in \mathcal{C}\left(c^{\prime}, d\right)$ to $Q(f) Q(s)^{-1}$. To show it is an isomorphism, we follow the strategy of [GZ67, pp. 13/14]. We consider a category $\Sigma^{-1} \mathcal{C}$ with the same objects as $\mathcal{C}$ and for $c, d \in \mathcal{C}$ the Hom set $\Sigma^{-1} \mathcal{C}(c, d)$ is given by the left hand side of (A.5.1). Using Axioms (ii) and (iii), we define for three objects $c, d, e \in \mathcal{C}$ a composition

$$
\underset{c^{\prime} \in \Sigma \downarrow c}{\lim _{f}} \mathcal{C}\left(c^{\prime}, d\right) \times \underset{d^{\prime} \in \Sigma \downarrow d}{\lim } \mathcal{C}\left(d^{\prime}, e\right) \rightarrow \underset{c^{\prime} \in \Sigma \downarrow c}{\lim } \mathcal{C}\left(c^{\prime}, e\right)
$$

which is shown to be well-defined and associative thanks to Axiom (iv). Now (A.5.1) yields a functor $\Sigma^{-1} \mathcal{C} \rightarrow \mathcal{C}\left[\Sigma^{-1}\right]$. But there is also an obvious functor $\mathcal{C} \rightarrow \Sigma^{-1} \mathcal{C}$ that is the identity on objects. (We use Axiom (i) to define the maps for the Hom sets.) It is easily seen to have the universal property of $\mathcal{C}\left[\Sigma^{-1}\right]$. Hence (A.5.1) is an isomorphism for all $(c, d)$.

c) The first statement has already been observed; the second one follows readily from (A.5.1).

Notation A.5.3. We shall write $\Sigma^{-1} \mathcal{C}$ instead of $\mathcal{C}\left[\Sigma^{-1}\right]$ if $\Sigma$ enjoys a calculus of fractions.

Corollary A.5.4. If $\sum$ admits a calculus of right fractions and if for any $c \in \mathcal{C}$, the category $\sum \downarrow c$ contains a small cofinal subcategory, then the Hom sets of $\Sigma^{-1} \mathcal{C}$ are small. 
Corollary A.5.5. Let $(\mathcal{C}, \Sigma)$ be a localiser such that $\sum$ enjoys a calculus of right fractions. Let $F: \mathcal{C} \rightarrow \mathcal{D}$ be a functor. Suppose that $F$ inverts the morphisms of $\sum$ and that, for any $c, d \in \mathcal{C}$, the obvious map

$$
\underset{c^{\prime} \in \Sigma \downarrow c}{\lim _{\longrightarrow}} \mathcal{C}\left(c^{\prime}, d\right) \rightarrow \mathcal{D}(F(c), F(d))
$$

is an isomorphism. Then the functor $\Sigma^{-1} F: \Sigma^{-1} \mathcal{C} \rightarrow \mathcal{D}$ induced by $F$ is fully faithful.

Proposition A.5.6.

a) Let $(\mathcal{C}, \Sigma)$ be a localiser. Assume that $\sum$ enjoys a calculus of right fractions. Then the localisation functor $Q: \mathcal{C} \rightarrow \Sigma^{-1} \mathcal{C}$ is left exact; if limits indexed by a finite category I exist in $\mathcal{C}$, they also exist in $\Sigma^{-1} \mathcal{C}$.

b) Let $\mathcal{C}$ be an essentially small category closed under finite limits, and let $G: \mathcal{C} \rightarrow \mathcal{D}$ be a left exact functor. Let $\Sigma=\{s \in \operatorname{Ar}(\mathcal{C}) \mid G(s)$ is invertible $\}$. Then $\sum$ enjoys a calculus of right fractions; the induced functor $\Sigma^{-1} \mathcal{C} \rightarrow \mathcal{D}$ is conservative and left exact.

Proof. After passing to the opposite categories, a) is [GZ67, Chapter I, Proposition 3.1 and Corollary 3.2] and b) is [GZ67, Chapter I, Proposition 3.4].

\section{A.6. Pro- $\sum$-objects}

Definition A.6.1. Let $(\mathcal{C}, \Sigma)$ be a localiser. We write $\operatorname{pro}_{\Sigma}-\mathcal{C}$ for the full subcategory of the category pro-C of pro-objects of $\mathcal{C}$ consisting of filtered inverse systems whose transition morphisms belong to $\Sigma$. An object of $\operatorname{pro}_{\Sigma}-\mathcal{C}$ is called a pro- $\sum$-object.

Proposition A.6.2. Suppose that $\sum$ has a calculus of right fractions and, for any $c \in \mathcal{C}$, the category $\Sigma \downarrow c$ contains a small cofinal subcategory. Then $Q: \mathcal{C} \rightarrow \Sigma^{-1} \mathcal{C}$ has a pro-left adjoint $Q^{!}$, which takes an object

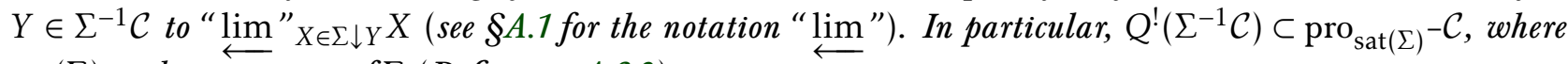
$\operatorname{sat}(\Sigma)$ is the saturation of $\Sigma$ (Definition A.3.2).

Proof. In view of Corollary A.5.4 and Proposition A.5.6, this follows from Proposition A.5.2 b).

Remark A.6.3. Consider the localisation functor $Q: \mathcal{C} \rightarrow \Sigma^{-1} \mathcal{C}$ : it has a left Kan extension

$$
\hat{Q}: \operatorname{pro}_{\text {sat }(\Sigma)}-\mathcal{C} \rightarrow \Sigma^{-1} \mathcal{C}
$$

$[$ Mac98, Chapter $\mathrm{X}]$ along the constant functor $\mathcal{C} \rightarrow \operatorname{pro}_{\text {sat }(\Sigma)}-\mathcal{C}$, given by the formula

$$
\hat{Q}\left(“ \lim ^{\longleftarrow} ” C_{\alpha}\right)=\underset{\lim }{\longleftarrow} Q\left(C_{\alpha}\right) \text {. }
$$

(The right hand side makes sense as an inverse limit of isomorphisms.) Then one checks easily that $Q !$ is left adjoint to $\hat{Q}$.

Theorem A.6.4. Let $(\mathcal{C}, \Sigma)$ be a localiser verifying the conditions of Proposition A.6.2. Let $Q: \mathcal{C} \rightarrow \Sigma^{-1} \mathcal{C}$ denote the localisation functor, and consider the string of adjoint functors $\left(Q_{!}, Q^{*}, Q_{*}\right)$ between $\hat{\mathcal{C}}$ and $\widehat{\Sigma^{-1} \mathcal{C}}$ from $\S A .4$. Then:

(1) $Q$ ! has a pro-left adjoint, and is therefore exact.

(2) For $F \in \hat{\mathcal{C}}$ and $Y \in \Sigma^{-1} \mathcal{C}$, we have

$$
Q_{!} F(Y)=\underset{X \in \sum \downarrow Y}{\lim _{\longrightarrow}} F(X) .
$$

Proof. This follows from Propositions A.4.1 a) and A.6.2.

If $(\mathcal{A}, \Sigma)$ is a localiser with $\mathcal{A}$ additive and $\Sigma$ enjoys a calculus of right fractions, then $\Sigma^{-1} \mathcal{A}$ is additive and so is the functor $Q: \mathcal{A} \rightarrow \Sigma^{-1} \mathcal{A}$ [GZ67, Chapter I, Corollary 3.3]. For future reference, we give the additive analogue of Theorem A.6.4 (see the paragraph before Proposition A.4.1 for $\operatorname{Mod}-\mathcal{A}$ ): 
Theorem A.6.5. Let $(\mathcal{A}, \Sigma)$ be a localiser; assume that $\mathcal{A}$ is an additive category and that $\Sigma$ has a calculus of right fractions. Let $Q: \mathcal{A} \rightarrow \Sigma^{-1} \mathcal{A}$ denote the localisation functor, as well as the string of adjoint functors $\left(Q_{!}, Q^{*}, Q_{*}\right)$ between $\operatorname{Mod}-\mathcal{A}$ and $\operatorname{Mod}-\Sigma^{-1} \mathcal{A}$. Then:

(1) $Q !$ has a pro-left adjoint, and is therefore exact.

(2) For $F \in \operatorname{Mod}-\mathcal{A}$ and $Y \in \Sigma^{-1} \mathcal{A}$, we have

$$
Q_{!} F(Y)=\underset{X \in \sum \downarrow Y}{\lim _{\longrightarrow}} F(X) .
$$

\section{A.7. cd-structures}

Let $\mathcal{C}$ be a category with an initial object. According to [Voe10a], a $c d$-structure on $\mathcal{C}$ is given by a collection of commutative squares stable under isomorphisms, called distinguished squares. Any cd-structure defines a topology on $\mathcal{C}$ : the smallest Grothendieck topology such that for a distinguished square of the form

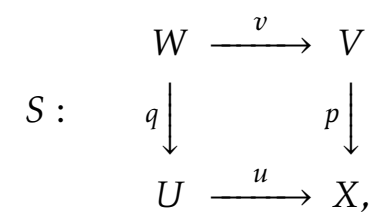

the sieve generated by the morphisms $\{p: V \rightarrow X, u: U \rightarrow X\}$ is a cover sieve and such that the empty sieve is a cover sieve of the initial object $\emptyset$.

Recall from [Voe10a] some important properties of cd-structures.

Definition A.7.1. Let $\mathcal{C}$ be a category with an initial object $\emptyset$.

(1) Let $P$ be a cd-structure on $\mathcal{C}$. The class $\mathcal{S}_{P}$ of simple covers is the smallest class of families of morphisms of the form $\left\{U_{i} \rightarrow X\right\}_{i \in I}$ satisfying the following two conditions:

- for any isomorphism $f,\{f\}$ is in $\mathcal{S}_{P}$

- for a distinguished square $Q$ of the form (A.7.1) and families $\left\{p_{i}: V_{i} \rightarrow V\right\}_{i \in I}$ and $\left\{q_{j}: U_{j} \rightarrow U\right\}_{j \in J}$ in $\mathcal{S}_{P}$ the family $\left\{p \circ p_{i}, u \circ q_{j}\right\}_{i \in I, j \in J}$ is in $\mathcal{S}_{P}$.

(2) A cd-structure on $\mathcal{C}$ is called complete if any cover sieve of an object $X \in \mathcal{C}$ which is not isomorphic to $\emptyset$ contains a sieve generated by a simple cover.

(3) A cd-structure $P$ is called regular if for $S \in P$ of the form (A.7.1) one has

- $S$ is a pullback square (i.e., is cartesian)

- $u$ is a monomorphism

- the morphisms of sheaves

$$
\Delta \bigsqcup \rho(v): \rho(V) \bigsqcup \rho(W) \times_{\rho(U)} \rho(W) \rightarrow \rho(V) \times_{\rho(X)} \rho(V)
$$

is surjective, where for $C \in \mathcal{C}$ we denote by $\rho(C)$ the sheaf associated with the presheaf represented by $C$, and $\Delta$ is induced by the diagonal map.

Lemma A.7.2 ([Voe10a, Lemma 2.5]). A cd-structure is complete provided:

(1) any morphism with values in $\emptyset$ is an isomorphism, and

(2) for any distinguished square $S$ of the form (A.7.1) and for any morphism $X^{\prime} \rightarrow X$, the square $S^{\prime}=X^{\prime} \times_{X} S$ is defined and distinguished.

Lemma A.7.3 ([Voe10a, Lemma 2.11]). A cd-structure is regular provided, for any distinguished square $S$ of the form (A.7.1) we have

(1) $S$ is cartesian, 
(2) $u$ is a monomorphism, and

(3) the objects $V \times_{X} V$ and $W \times_{U} W$ exist in $\mathcal{C}$ and the derived square

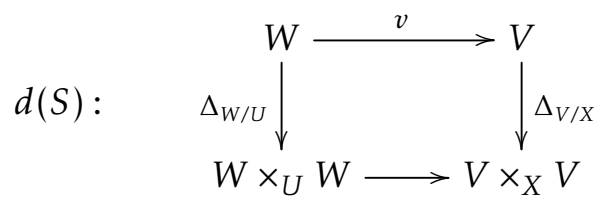

is distinguished.

Definition A.7.4. A cd-structure verifying the conditions of Lemma A.7.2 (resp. A.7.3) is called strongly complete (resp. strongly regular).

Remark A.7.5. The square (A.7.2) is cartesian. This is a formal consequence of Lemma A.7.3, since any distinguished square with respect to a regular cd-structure is cartesian by definition. However, there is a more direct proof: let $Z \stackrel{a}{\rightarrow} V$ and $Z \stackrel{b}{\rightarrow} W \times_{U} W$ be two morphisms making the corresponding square commute. Then $b$ amounts to two morphisms $b_{1}, b_{2}: Z \rightarrow W$ such that (with the notation of (A.7.1)) $q b_{1}=q b_{2}$ and $a=v b_{1}=v b_{2}$. Since $S$ is cartesian by (1), we have $b_{1}=b_{2}: Z \rightarrow W$, which is a solution to the universal problem.

Proposition A.7.6. Let $(\mathcal{C}, \Sigma)$ be a localiser such that $\Sigma$ admits a calculus of right fractions.

(1) IfC has an initial object verifying Conditon (1) of Lemma A.7.2, so does $\Sigma^{-1} \mathcal{C}$.

(2) Assume (1) and let $Q: \mathcal{C} \rightarrow \Sigma^{-1} \mathcal{C}$ be the localisation functor. Suppose given a cd-structure $P$ on $\mathcal{C}$, and let $P^{\prime}$ be the cd-structure on $\Sigma^{-1} \mathcal{C}$ given by all squares isomorphic to a square of the form $Q(S)$, where $S \in P$. If $P$ is strongly complete (resp. strongly regular), so is $P^{\prime}$.

Proof. (1) Let $\emptyset$ be an initial object of $\mathcal{C}$. Since $Q$ is (essentially) surjective, $Q(\emptyset)$ admits a morphism to any object; Condition (1) of Lemma A.7.2 for $\emptyset$ implies that this morphism is unique, and this in turn implies the same condition for $Q(\emptyset)$.

(2) By Proposition A.5.6 a), $Q$ commutes with finite limits. This implies Condition (2) of Lemma A.7.2. Conditions (1), (3) of Lemma A.7.3 for $P^{\prime}$ follow from the same conditions for $P$ (note that the diagonals are preserved by $Q$, since they are finite limits). It remains to show that $Q$ carries a monomorphism $u: U \rightarrow X$ to a monomorphism. Let $f, g: V \rightarrow U$ be two morphisms in $\Sigma^{-1} \mathcal{C}$ such that $Q(u) f=Q(u) g$. By calculus of fractions, we may write $f=Q(\tilde{f}) Q(s)^{-1}$ and $g=Q(\tilde{g}) Q(s)^{-1}$ for some $\tilde{f}, \tilde{g} \in \operatorname{Ar}(\mathcal{C})$ and $s \in \Sigma$. Then $Q(u \tilde{f})=Q(u \tilde{g})$. By Proposition A.5.2 c), we may find $t \in \sum$ such that $u \tilde{f} t=u \tilde{g} t$, which implies $\tilde{f} t=\tilde{g} t$ since $u$ is a monomorphism. This shows $f=g$, as desired.

\section{A.8. A pull-back lemma}

We shall use the following elementary lemma several times.

Lemma A.8.1. Let $\mathcal{C}, \mathcal{D}$ be abelian categories and let $\mathcal{C}^{\prime} \subset \mathcal{C}, \mathcal{D}^{\prime} \subset \mathcal{D}$ be full abelian subcategories. Let $\mathcal{c}: \mathcal{C} \rightarrow \mathcal{D}$ and $c^{\prime}: \mathcal{C}^{\prime} \rightarrow \mathcal{D}^{\prime}$ be additive functors satisfying $c i_{C}=i_{D} c^{\prime}$, where $i_{C}: \mathcal{C}^{\prime} \rightarrow \mathcal{C}$ and $i_{D}: \mathcal{D}^{\prime} \rightarrow \mathcal{D}$ are inclusion functors.

(1) If c is faithful, so is $c^{\prime}$.

(2) Suppose that $i_{D}$ is strongly additive or has a strongly additive left inverse (for example, a left adjoint). If $c$ and $i_{C}$ are strongly additive, so is $c^{\prime}$.

(3) Suppose that $i_{C}$ has a left adjoint $a_{C}$. If $c$ has a left adjoint $d$, then $d^{\prime}=a_{C} d i_{D}$ is a left adjoint of $c^{\prime}$. If $d$ and $a_{\mathcal{C}}$ are exact, so is $d^{\prime}$. Moreover, $a_{\mathcal{C}} d=d^{\prime} a_{\mathcal{D}}$ if $i_{\mathcal{D}}$ has a left adjoint $a_{\mathcal{D}}$.

(4) Suppose that $i_{C}$ and $i_{D}$ have left adjoints $a_{C}$ and $a_{D}$, that $a_{D}$ is exact, and that $a_{D} c=c^{\prime} a_{C}$. If $c$ is exact, then so is $c^{\prime}$. 
Proof. (1) is obvious. (2) Let $\left\{F_{i}\right\}_{i \in I}$ be a family of objects of $\mathcal{C}^{\prime}$. We must show that the natural map

$$
f: \bigoplus_{i \in I} c^{\prime}\left(F_{i}\right) \rightarrow c^{\prime}\left(\bigoplus_{i \in I} F_{i}\right)
$$

is an isomorphism. The composition

$$
\bigoplus_{i \in I} i_{D} c^{\prime} F_{i} \stackrel{g}{\rightarrow} i_{D}\left(\bigoplus_{i \in I} c^{\prime}\left(F_{i}\right)\right) \stackrel{i_{D} f}{\longrightarrow} i_{D} c^{\prime}\left(\bigoplus_{i \in I} F_{i}\right)
$$

is an isomorphism by the strong additivity of $c$ and $i_{C}$. If $i_{D}$ is strongly additive, $g$ is also an isomorphism and we are done. If now $i_{D}$ has a strongly additive left inverse $a_{D}$, we apply it to the diagram and get a composition

$$
\bigoplus_{i \in I} a_{D} i_{D} c^{\prime} F_{i} \stackrel{\sim}{\rightarrow} a_{D} \bigoplus_{i \in I} i_{D} c^{\prime} F_{i} \stackrel{a_{D} g}{\longrightarrow} a_{D} i_{D}\left(\bigoplus_{i \in I} c^{\prime}\left(F_{i}\right)\right) \stackrel{a_{D} i_{D} f}{\longrightarrow} a_{D} i_{D} c^{\prime}\left(\bigoplus_{i \in I} F_{i}\right)
$$

which is an isomorphism and naturally isomorphic to $f$. This concludes the proof of (2).

(3) For $F \in \mathcal{C}^{\prime}$ and $G \in \mathcal{D}^{\prime}$, we have $\mathcal{C}^{\prime}\left(a_{C} d i_{D} F, G\right)=\mathcal{D}\left(i_{D} F, c i_{C} G\right)=\mathcal{D}\left(i_{D} F, i_{D} c^{\prime} G\right)=\mathcal{D}^{\prime}\left(F, c^{\prime} G\right)$. This proves the first claim; therefore if $d$ and $a_{\mathcal{C}}$ are exact, $d^{\prime}$ is left exact, hence exact since it is right exact as a left adjoint. The last isomorphism follows from taking left adjoints of the isomorphism $c i_{\mathcal{C}}=i_{\mathcal{D}} c^{\prime}$.

(4) Let us take an exact sequence $0 \rightarrow F \rightarrow G \rightarrow H \rightarrow 0$ in $\mathcal{C}^{\prime}$. Put $K:=\operatorname{Coker}\left(i_{C} G \rightarrow i_{C} H\right) \in \mathcal{C}$. Since $a_{D} c K=c^{\prime} a_{C} K=0$, we get an exact sequence $0 \rightarrow a_{D} c i_{C} F \rightarrow a_{D} c i_{C} G \rightarrow a_{D} c i_{C} H \rightarrow 0$ by the exactness of $c$ and $a_{D}$. Using $a_{D} c=c^{\prime} a_{C}$ and $a_{C} i_{C}=\operatorname{Id}$ (Lemma A.3.1), we conclude $0 \rightarrow c^{\prime} F \rightarrow c^{\prime} G \rightarrow c^{\prime} H \rightarrow 0$ is exact.

The proof of Lemma A.8.1 (2) implicitly used the following (trivial) lemma, which we state for the sake of clarity.

Lemma A.8.2. Let $\mathcal{D} \subseteq \mathcal{C}$ be a full embedding of categories. Suppose that a direct (resp. inverse) system $\left(d_{\alpha}\right)$ of objects of $\mathcal{D}$ has a colimit (resp. a limit) in $\mathcal{C}$, which is isomorphic to an object $d$ of $\mathcal{D}$. Then $d$ represents the (co)limit of $\left(d_{\alpha}\right)$ in $\mathcal{D}$.

\section{A.9. Homological algebra}

Recall Grothendieck's theorem [Gro57, théorème 2.4.1]:

Theorem A.9.1. Let $\mathcal{A} \stackrel{F}{\rightarrow} \mathcal{B} \stackrel{G}{\rightarrow} \mathcal{C}$ be a string of left exact functors between abelian categories. Suppose that $\mathcal{A}$ and $\mathcal{B}$ have enough injectives and that $F$ carries injectives of $\mathcal{A}$ to $G$-acyclics. Then, for any $A \in \mathcal{A}$, there is a convergent spectral sequence

$$
E_{2}^{p, q}=R^{p} G R^{q} F(A) \Rightarrow R^{p+q}(G F)(A) .
$$

Examples A.9.2. If $F$ has an exact left adjoint, it carries injectives to injectives. If $G$ is exact, the hypothesis on $F$ is automatically verified.

The following is a slight generalization of [Mil80, Chapter III, Proposition 2.12], (where the underlying category of $\mathcal{S}$ is supposed to be a category of schemes).

Lemma A.9.3. Let $F$ be a sheaf of abelian groups on a site $\mathcal{S}$. The following conditions are equivalent.

(1) We have $H^{q}(X, F)=0$ for any $X \in \mathcal{S}$ and $q>0$.

(2) We have $\check{H}^{q}(X, F)=0$ for any $X \in \mathcal{S}$ and $q>0$.

(3) We have $\check{H}^{q}(U / X, F)=0$ for any cover $U \rightarrow X$ in $\mathcal{S}$ and $q>0$.

(4) The sheaf $F$ is $i_{\mathcal{S}}$-acyclic, where $i_{\mathcal{S}}$ is the inclusion functor of the category of sheaves to that of presheaves. 
Proof. For $X \in \mathcal{S}$, we write $\Gamma_{X}$ (resp. $\Gamma_{X}^{\mathrm{pr}}$ ) for the functor $F \mapsto F(X)$ from the category of sheaves (resp. presheaves) to Ab. We have $\Gamma_{X}=\Gamma_{X}^{\mathrm{pr}} i_{\mathcal{S}}$. Since $\Gamma_{X}^{\mathrm{pr}}$ is exact, Theorem A.9.1 implies $R^{q} \Gamma_{X}=\Gamma_{X}^{\mathrm{pr}} R^{q} i_{\mathcal{S}}$, and hence $H^{q}(X, F)=R^{q} i_{\mathcal{S}} F(X)$. This proves the equivalence of (1) and (4). The rest is shown in the same way as [Mil80, Chapter III, Proposition 2.12].

Definition A.9.4. We say $F$ is flabby if the conditions of Lemma A.9.3 are satisfied.

Lemma A.9.5. Let $\mathcal{S}$ be the category of abelian sheaves on a site $\mathcal{C}, \mathcal{T}$ an abelian category, and $c^{*}: \mathcal{T} \rightarrow \mathcal{S}$ an additive functor which has a left adjoint $c_{!}: \mathcal{S} \rightarrow \mathcal{T}$. Suppose that any cover in $\mathcal{C}$ admits a refinement $U \rightarrow X$ such that $c_{!}(\check{C}(U / X))$ is exact in $\mathcal{T}$, where

$$
\check{C}(U / X)=\left(\cdots \rightarrow y\left(U \times_{X} U\right) \rightarrow y(U) \rightarrow y(X) \rightarrow 0\right)
$$

is the Cech complex associated to $U \rightarrow X$ (y denotes the Yoneda functor). Then $c^{*} I$ is flabby for any injective object $I \in \mathcal{T}$.

Proof. (Compare [Voe00, Proposition 3.1.7].) It suffices to show $\check{H}^{q}\left(U / X, c^{*} I\right)=0$ for any $q>0$ and for any $U \rightarrow X$ as in the assumption. If we denote by $U_{X}^{n}$ the $n$-fold fiber product of $U$ over $X$, then $\check{H}^{q}\left(U / X, c^{*} I\right)$ is computed as the cohomology of the complex

$$
c^{*} I\left(U_{X}^{\bullet+1}\right)=\mathcal{S}\left(y\left(U_{X}^{\bullet+1}\right), c^{*} I\right)=\mathcal{T}\left(c_{!} y\left(U_{X}^{\bullet+1}\right), I\right),
$$

which is acyclic by the assumption and the injectivity of $I$.

\section{A.10. Grothendieck categories}

Recall that a Grothendieck abelian category (for short, a Grothendieck category) is an abelian category verifying Axiom AB5 of [Gro57]: small colimits are representable and exact, and having a set of generators (equivalently, a generator). These generators are generators by strict epimorphisms. We have the following basic facts:

\section{Theorem A.10.1.}

a) Any Grothendieck category is complete and has enough injectives.

b) Let $\mathcal{F}: \mathcal{C} \rightarrow \mathcal{D}$ be a functor, where $\mathcal{C}$ is a Grothendieck category. Then $F$ has a right adjoint if and only if it commutes with all colimits.

c) Let $\mathcal{C}$ be a Grothendieck category, $\mathcal{B} \subset \mathcal{C}$ be a Serre subcategory, $\mathcal{D}=\mathcal{C} / \mathcal{B}$ and $G: \mathcal{C} \rightarrow \mathcal{D}$ the (exact) localisation functor. Then $G$ has a right adjoint $D$ if and only if $\mathcal{B}$ is stable under infinite direct sums. In this case, $\mathcal{B}$ and $\mathcal{D}$ are Grothendieck.

d) Let $G: \mathcal{C} \leftrightarrows \mathcal{D}: D$ be a pair of adjoint additive functors between additive categories, with $D$ fully faithful. IfC is Grothendieck and $G$ is exact, $\mathcal{D}$ is Grothendieck.

Proof. a) See [Gro57, théorème 1.10.1], [SGA4, exposé V, remarque 0.2.1] or [KS06, Theorem 8.3.27 (i) and 9.6.2]. b) See [KS06, Proposition 8.3.27 (iii)]. c) See [Gab62, chapitre III, proposition 8 and 9]. d) Let $\mathcal{B}$ be the kernel of $G$. Then $\mathcal{B}$ is easily seen to be a Serre subcategory (e.g. [Gab62, chapitre III, proposition 5]), so the claim follows from c).

Theorem A.10.2. For any additive category $\mathcal{A}, \operatorname{Mod}-\mathcal{A}$ is a Grothendieck category with a set of projective generators.

Proof. See e.g. [AK02, Proposition 1.3.6] for the first statement; the projective generators are given by $\mathcal{E}=\{y(A) \mid A \in \mathcal{A}\}$. 


\section{References}

[AK02] Y. André, B. Kahn, Nilpotence, radicaux et structures monoïdales (with an appendix by P. O'Sullivan), Rend. Sem. Math. Univ. Padova 108 (2002), 107-291.

[AM69] M. Artin, B. Mazur, Étale homotopy, Lect. Notes in Math. 100, Springer, 1969.

[Ayo15] J. Ayoub, Motifs de variétés analytiques rigides, Mém. SMF 140-141 (2015), vi+386.

[BE03] S. Bloch, H. Esnault, An additive version of higher Chow groups, Ann. Sci. Éc. Norm. Sup. 36 (2003), no. 3, 463-477.

[BM08] E. Bierstone, P. Milman, Functoriality in resolution of singularities, Publ. RIMS, Kyoto Univ. 44 (2008), 609-639.

[BS19] F. Binda, S. Saito, Relative cycles with moduli and regulator maps, J. Inst. Math. Jussieu 18 (2019), no. 6, 1233-1293.

[Gab62] P. Gabriel, Des catégories abéliennes, Bull. SMF 90 (1962), 323-448.

[GZ67] P. Gabriel, M. Zisman, Calculus of fractions and homotopy theory, Springer, 1967.

[Gro57] A. Grothendieck, Sur quelques points d'algèbre homologique, Tohôku Math. J. 9 (1957), 119-221.

[Har77] R. Hartshorne, Algebraic Geometry, Springer, 1977.

[KMSY20] B. Kahn, H. Miyazaki, S. Saito, T. Yamazaki, Motives with modulus, III: The categories of motives, preprint arXiv:2011.11859 (2020).

[KMSY21] _ _ Motives with modulus, II: Modulus sheaves with transfers for proper modulus pairs, Épijournal de Géométrie Algébrique 5 (2021), article 2.

[KP12] A. Krishna, J. Park, Moving lemma for additive higher Chow groups, Algebra Number Theory 6 (2012), no. 2, 293-326.

[KS06] M. Kashiwara, P. Schapira, Categories and sheaves, Springer, 2006.

[KSY16] B. Kahn, S. Saito, T. Yamazaki, Reciprocity sheaves (with two appendices by Kay Rülling), Compositio Math. 152 (2016), 1851-1898.

[KSY15] _ Motives with modulus, preprint arXiv:1511.07124 (2015), withdrawn.

[KSY17] — Reciprocity sheaves, II, preprint arXiv:1707.07398 (2017).

[MVW06] C. Mazza, V. Voevodsky, C. Weibel, Lecture notes on motivic cohomology, Clay Math. Monographs 2, AMS, 2006.

[Mac98] S. Mac Lane, Categories for the working mathematician, Grad. Texts in Math. 5, Springer (2nd ed.), 1998.

[Mil80] J. Milne, Étale cohomology, Princeton Mathematical Series 33. Princeton University Press, Princeton, N.J., 1980.

[Miy19] H. Miyazaki, Cube invariance of higher Chow groups with modulus, J. Algebraic Geom. 28 (2019), no. 2, 339-390.

[Par09] J. Park, Regulators on additive higher Chow groups, Amer. J. Math. 131 (2009), 257-276.

[RG71] M. Raynaud, L. Gruson, Critères de platitude et de projectivité, Invent. Math. 13 (1971), 1-89.

[Rio02] J. Riou, Théorie homotopique des S-schémas, mémoire de DEA, Paris 7, 2002. Available at http://www.math.u-psud.fr/ riou/dea/dea.pdf. 
[Rül07] K. Rülling, The generalized de Rham-Witt complex over a field is a complex of zero-cycles, J. Algebraic Geom. 16 (2007), no. 1, 109-169.

[Sai20] S. Saito, Purity of reciprocity sheaves, Adv. Math. 366 (2020), 107067 j.aim.2020.107067.

[SV00] A. Sulin, V. Voevodsky, Bloch-Kato Conjecture and motivic cohomology with finite coefficients, in : The Arithmetic and Geometry of Algebraic Cycles. eds. B. Gordon, J. Lewis, S. Müller-Stach, S. Saito, N. Yui, NATO Science Series 548, 2000.

[Voe00] V. Voevodsky, Triangulated categories of motives over a field, in : Cycles, transfers and motivic cohomology theories, by E. Friedlander, A. Suslin, V. Voevodsky, Ann. Math. Studies 143, Princeton University Press, 2000, 188-238.

[Voe10a] Homotopy theory of simplicial sheaves in completely decomposable topologies, J. pure appl. Algebra 214 (2010), 1384-1398.

[Voe10b] U Unstable motivic homotopy categories in Nisnevich and cdh-topologies, J. pure appl. Algebra 214 (2010) 1399-1406.

\section{Acronyms}

[EGA3] A. Grothendieck, Éléments de géométrie algébrique. III. Étude cohomologique des faisceaux cohérents. I., Inst. Hautes Études Sci. Publ. Math. No. 11 (1961), 167 pp.

[SGA1] A. Grothendieck et al, Revêtements étales et groupe fondamental (SGA 1), Séminaire de Géométrie Algébrique du Bois-Marie 1960 - 1961, new edition: Documents mathématiques 3, SMF, 2003.

[SGA3] M. Demazure, A. Grothendieck, Schémas en groupes, new edition, SMF, 2012.

[SGA4] E. Artin, A. Grothendieck, J.-L. Verdier, Théorie des topos et cohomologie étale des schémas (SGA4), Lect. Notes in Math. 269, 270, 305, Springer, 1972-73. 